\title{
The Trainset Railroad Simulation
}

Richard A. Brown (Editor)*

Fred B. Schneider (Editor)

Jacob Aizikowitz ${ }^{\star \star}$

Thomas C. Bressoud

Tony Lekas ${ }^{\star \star \star}$

TR 93-1329

February 1993

Department of Computer Science Cornell University

Ithaca, NY 14853-7501

"Department of Mathematics, St. Olaf College, Northfield, MN 55057

**Electronics for Imaging, 950 Elm Avenue, San Bruno, CA 94066

***Digital Equipment Corporation, 9 Northeastern Boulevard, Salem, NH 03079 


\title{
The Trainset Railroad Simulation
}

\author{
Richard A. Brown ${ }^{1}$ and Fred B. Schneider, Editors \\ Jacob Aizikowitz ${ }^{2}$ Thomas C. Bressoud Tony Lekas ${ }^{3}$
}

The RR Project

Department of Computer Science

Cornell University

Ithaca, NY 14850

February 13, 1993

\footnotetext{
${ }^{1}$ Department of Mathematics, St. Olaf College, Northfield, MN 55057

${ }^{2}$ Electronics for Imaging, $950 \mathrm{Elm}$ Avenue, San Bruno, CA 94066

${ }^{3}$ Digital Equipment Corporation, 9 Northeastern Boulevard, Salem, NH 03079
} 


\section{.}


Copyright (C) 1993 Richard A. Brown and Fred B. Schneider.

All rights reserved.

Permission to copy in whole or in part without payment of fee is granted for nonprofit educational and research purposes provided that all such whole or partial copies include the following: a notice that such copying is by permission of the copyright owners; an acknowledgement of the authors and individual contributors to the work; and all applicable portions of the copyright notice. Copying, reproducing or republishing in whole or in part for any commercial purpose or for other purpose is prohibited without written permission of the copyright owners. 



\section{Contents}

Preface iv

0 Introduction and Overview 1

Obtaining a Copy . . . . . . . . . . . . . . . 2

1 Trainset User's Guide 3

1.1 Getting Started . . . . . . . . . . . . . . . . . 3

1.2 How to Build a Layout of Blocks and Trains . . . . . . . . . 10

1.3 How to Run a Layout . . . . . . . . . . . . . . . . . . . . 21

1.4 Control Programs . . . . . . . . . . . . . . . . . . . . . . 21

2 Trainset Railroads $\quad 30$

2.1 Introduction . . . . . . . . . . . . . . . . 30

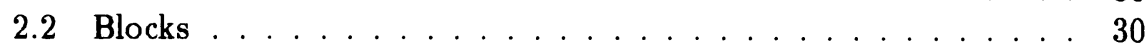

2.3 Trains . . . . . . . . . . . . . . . . 35

3 Automatic Control Interface (ACI) 38

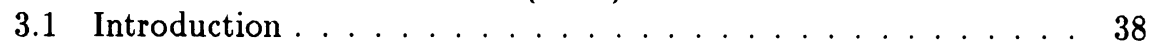

3.2 Initial-State Download . . . . . . . . . . . . . . . . . 39

3.3 Commands . . . . . . . . . . . . . . . . . . . . . 40

3.4 Queries . . . . . . . . . . . . . . . . . . 43

3.5 Voting . . . . . . . . . . . . . . . . . . . 45

3.6 Timer Facilities . . . . . . . . . . . . . . . . . . . . . 47

4 Low-Level Interface (LLI) $\quad 50$

4.1 Introduction . . . . . . . . . . . . . . . . . 50

4.2 Messages . . . . . . . . . . . . . . . . . . 50

4.3 Initiating Communication $\ldots \ldots \ldots \ldots \ldots \ldots$

4.4 Initial-State Download . . . . . . . . . . . . . . . . 53

4.5 Commands and Queries . . . . . . . . . . . . . . . 53

4.6 Quit Message From the Simulator . . . . . . . . . . . . . . . 54

A Constants Used by Trainset $\quad 55$ 
B Code Listings: ACI $\quad 56$

B.1 aci.h, Interface Header File . . . . . . . . . . . . . . 56

B.2 acitest.c, Example Using the ACI Interface . . . . . . . . . 60

C Code Listings: LLI $\quad \mathbf{7 5}$

C.1 cpi.h, Interface Header File . . . . . . . . . . . . . . . 75

C.2 aci.c, Example Using the LLI Interface . . . . . . . . . . . . . . 83

$\begin{array}{ll}\text { D Reference Pages } & 97\end{array}$ 


\section{Preface}

I became intrigued with real-time systems in the spring of 1986 . Here was an application domain where using formal methods is justified, because the cost in life and property of programmer errors could be so great. Here also was an application domain where making assumptions about hardware failure modes is inappropriate, and so a system must be able to tolerate so-called Byzantine failures. My research efforts had been concerned with these two subjects, thus putting me in the enviable position of having discovered a "problem" for my various "solutions".

Attacking a real process-control problem seemed like a good way to get a better understanding of the area. But, which problem? The problem had to be simple enough so that Computer Science issues dominated any applicationdependent details. Yet, the problem could not be too simple or else some key aspect of real-time systems might be overlooked. I was aware that various research groups (e.g., at University of Newcastle upon Tyne and at University of Waterloo) had used electric toy trains as a vehicle [sic] for such research, and so that was an obvious place to start. After studying a bit of railroading, however, it became clear that toy trains are not accurate models of reality they change the problem too much. For example, real trains cannot accelerate or decelerate as rapidly as toy trains do. Therefore, a control program for a real train must anticipate changes to train speed; a control program for a toy train need not. Some of the inaccuracies of toy trains can be corrected by modifying the electronics used to control the trains, but dealing with this and the other custom hardware necessary for integrating a toy train set with a computer system seemed like a black hole (as only custom hardware can be) that I should avoid. Thus, I decided to build a railroad simulator along with support software for constructing railroad layouts, controlling those layouts from networks of computers, and monitoring such control experiments.

After the first version of the railroad simulator was built, it became clear that my circumstances were not unique. Other scientists were also becoming interested in studying real-time programming issues and they, too, felt that a prototype application could be a useful research tool. Courses on real-time systems would benefit from using this software in laboratory exercises, particularly because specialized hardware and software were not required. So, we cleaned- 
up the code, wrote some user documentation, and put together this software distribution.

The Trainset system, as our railroad simulation software is now known, is the work of many people over the last 5 years. Jacob Aizikowitz defined our model of railroads and wrote the first railroad simulator in the spring of 1987; it ran under SUNOS Unix. Jacob also supervised MEng. students Ellen Blood, Anthony Pellegrini, and Jane Smidesang in producing an X10 graphics interface to the system. Michael Abbott, an undergraduate, then defined and implemented a high-level interface to the simulator for use by control programs. This software was then rewritten and ported to VMS, ULTRIX, and X11/DECWindows by Tony Lekas, a DEC engineer working with us as part of a DEC-funded research project at Cornell. Dick Brown joined the project in Fall 1989, spending that year and the following spring term on a major rewrite of the system and writing documentation for what we had. Dick was assisted by Thomas Bressoud, who rewrote and documented the layout editor and part of the graphics monitor software. Most recently, Donald Wihardja has helped us debug the installation procedure.

This software development effort would not have been possible without financial support from a number of sources. My research in concurrent and distributed systems has been funded by grants from the National Science Foundation since 1978 and from the Office of Naval Research since 1985. Dr. Andre van Tilborg, now the division director for Computer Science at ONR, was especially supportive as my ONR program manager during the initial stages of this project, and Gary Koob, his successor, has continued that tradition. Funding from Digital Equipment Corporation was also critical to the success of this project. Ed Balkovich of DEC encouraged me to apply for funding under Digital's External Research Program (ERP) and then served as our corporate liaison, helping to transfer our research results to engineers at DEC who could benefit from them. The DEC ERP funds allowed us to procure hardware for the laboratory used to develop this software and to run real-time systems experiments. John Gannon, the Software Engineering Program manager at NSF for 1988-89, alerted me to the NSF Software Capitalization Initiative and encouraged me to apply. Funding from that program supported Dick Brown's stay at Cornell and is largely responsible for transforming our research prototype into a system that could be widely distributed.

Fred B. Schneider Ithaca, New York 


\section{Chapter 0}

\section{Introduction and Overview}

Trainset is a real-time simulation of a railroad. The software consists of a simulator, an interactive graphics editor for defining railroad layouts and graphics monitor programs for displaying the state of the railroad and manually controlling it. Two communications interfaces to the simulator are provided.

- The control program interface (CPI) is used by computer programs that control Trainset railroads. The CPI consists of library routines and data structures, collectively called the ACI (Automatic Control Interface), and low-level message formats and related facilities, called the LLI (Low-Level Interface).

- The monitor interface is used only by the graphics monitor programs.

These interfaces can be in use simultaneously.

Trainset has been implemented in C language on Digital Equipment Corp. Ultrix, using DECwindows/X-11 and TCP/IP or DECnet.

This manual introduces Trainset and gives specifications for the railroads it implements. The document also discusses the mechanics of using Trainset and provides other information that a researcher or student should know in order to write control programs that interact with Trainset railroad layouts.

The manual is organized as follows.

Chapter 1 is a tutorial on using the software. It includes instructions for running a demonstration, creating and simulating a railroad layout and writing programs to control a Trainset railroad. A simple programming example is discussed to illustrate the use of the $\mathrm{ACl}$.

Chapter 2 specifies the attributes of simulated railroads that Trainset supports.

Chapter 3 discusses the ACI. A high-level mechanism is presented for establishing a connection with a running Trainset railroad simulation and receiving an initial-state download of that railroad. Commands and queries are described 
for interacting with a Trainset railroad. Utility routines that provide timer facilities are introduced, and a service is discussed that supports the writing of fault-tolerant control programs.

Chapter 4 documents the LLI. This information will be of interest to programmers who wish to bypass the ACI layer or reimplement it for other environments.

The appendices include reference pages for the programs that comprise Trainset and selected code listings.

A separate installation guide accompanies the software distribution.

\section{Obtaining a Copy}

You may obtain a copy of the Trainset software, installation instructions and the text of this manual from either of the Internet sites listed below.

- Itp.cs. cornell. edu (Cornell University)

- ftp.stolaf .edu (St. Olaf College)

In either case, use the file-transfer program $f$ tp to open a connection to the desired host. Use the login name anonymous, and provide your own Internet address as the password.

After logging in, issue the $\mathrm{ftp}$ command $\mathrm{cd}$ pub/trainset to access the Trainset distribution directory. Among the files in that directory are:

- README, which briefly describes Trainset and the contents of the distribution directory,

- install.dvi, installation manual for Trainset (in $\mathrm{TE}_{\mathrm{E}} \mathrm{X}$ output format),

- ts.dvi, the text of this manual, ${ }^{1}$ and

- trainset.tar.z, the source code package for Trainset.

See the manual page for $f t p(1)$ for file transfer instructions. Use $f t p$ file type binary for . $d v i$ and .tar. $z$ files. The source code package trainset.tar.z may be unpacked on most Unix systems by issuing the following command:

\% zcat trainset.tar.z | tar xve -

See the manual pages for compress(1) and $\operatorname{tar}(1)$ for more information about this unpacking procedure.

\footnotetext{
${ }^{1}$ To print this manual complete with figures, install Trainset at your local site and follow the printing instructions provided in the installation manual.
} 


\section{Chapter 1}

\section{Trainset User's Guide}

\section{$1.1 \quad$ Getting Started}

Trainset consists of programs to support interaction with a simulated railroad. Users can control the railroad manually and watch it in action by using graphics monitor programs. In addition, computer programs, called control programs, may be written to control a Trainset railroad.

The rest of this section gives you a chance to get acquainted with Trainset. In subsequent sections we explain how to create and run your own railroad layout and how to use the ACI library.

\subsubsection{Invoking the Programs}

To start the Trainset software, enter the following command.

\section{$\%$ ts}

(The symbol \% is assumed to be your shell prompt.) Three windows will open on your display, as shown in Figure 1.1. One of these, the Simulator Window, is a terminal window that displays messages from the simulation. The other two windows make up the graphics monitor. The Viever window shows the current positions of the railroad tracks and trains in a simulation. The Control Panel window has controls and indicators, including pushbuttons and slide bars; it is a graphical interface for controlling trains and switch blocks manually. ${ }^{1}$

The sample railroad layout displayed in the Viewer window of Figure 1.1 has two trains and 24 blocks. $^{2}$ Each train has two ends; one is called the head

\footnotetext{
${ }^{1}$ The names of the programs that comprise Trainset are $t s i m$ for the simulator, $t s v i e$ for the Vierer, tspanel for the Control Panel and tsed for the layout editor. tsed is discussed in Section 1.2 below.

${ }^{2} \mathrm{~A}$ careful reader may observe that the 24 block identifiers in Figure 1.1 range from 1 to 5 and 7 to 25 . Identifier 6 is associated with a subblock of the cross block cr 5 , as explained in Section 2.2.3.
} 


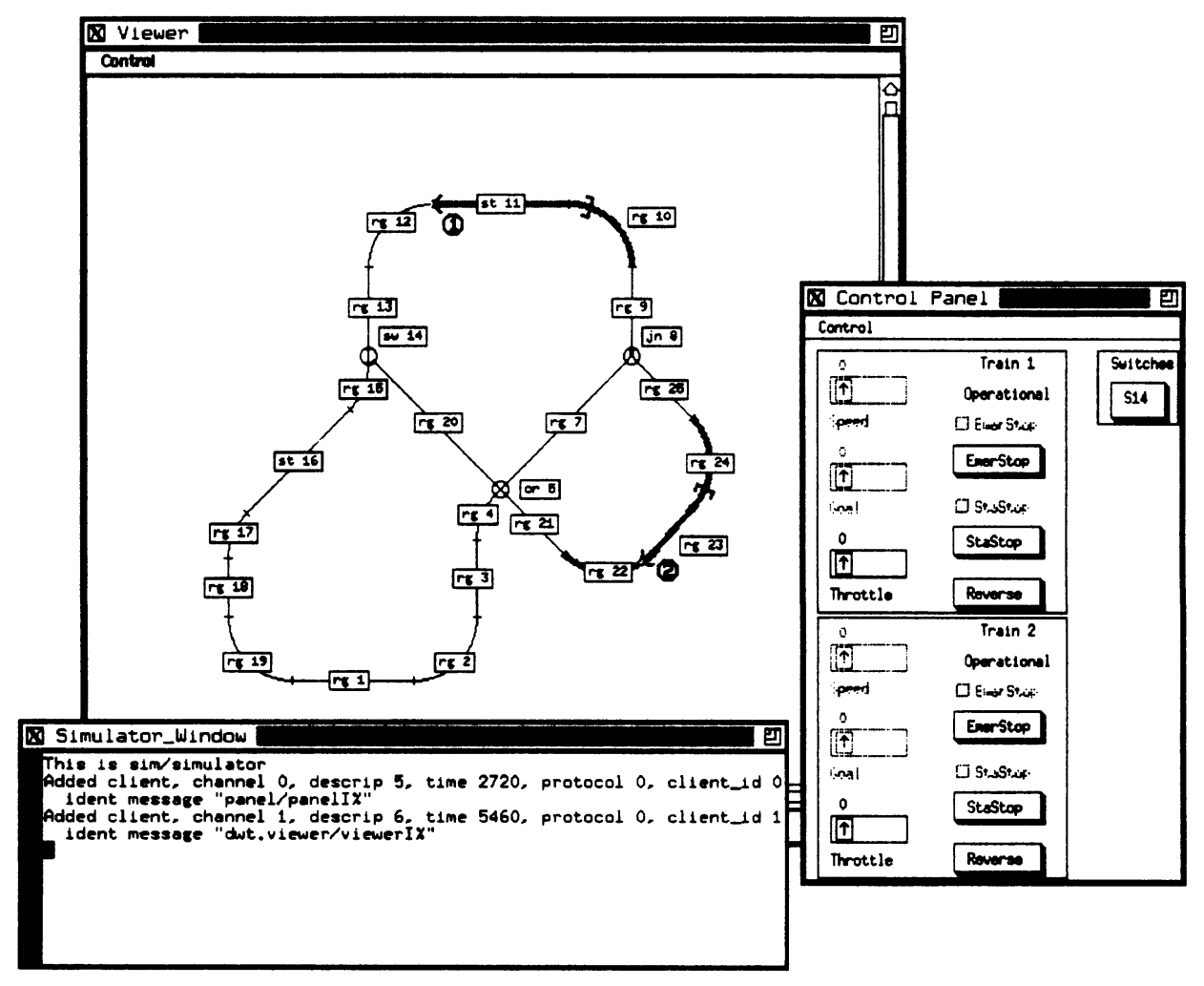

Figure 1.1: Sample railroad layout 
(indicated by an angle bracket) and the other is called the tail (indicated by a square bracket). A block's type (see Section 2.2) is defined by its label:

rg for regular blocks,

st for station blocks,

jn for join blocks,

cr for cross blocks and

sw for switch blocks.

Most blocks in the layout of Figure 1.1 are represented by straight lines or arcs, e.g., those labelled rg 1, rg 2 and st 11 . Five such blocks are thickened to indicate that they are occupied by a train. Three blocks (cr 5, jn 8 and sw 14) have other block types that are represented by circled symbols in the layout.

The control panel comprises one subwindow for each train and a pushbutton for each switch block. Each switch block's pushbutton can toggle that switch block's setting between the straight and turned settings. Each train's subwindow includes the following controls and indicators.

- Two slide bars labelled Speed and Goal that display the train's current speed and the goal speed desired for that train.

- A slide bar labelled Throttle for setting a new goal speed. An operational train accelerates or decelerates when its goal speed differs from its current speed.

- Two labels showing the name of the train (e.g., Train 1) and its state (Operational, Derailed or Collided).

- Two indicator lights, EmerStop and StaStop. The EmerStop light is illuminated while the train is performing an emergency stop. The StaStop light is illuminated when the train is capable of performing a station stop, described later.

- Three pushbuttons, EmerStop, StaStop and Reverse. The EmerStop pushbutton can be used to begin an emergency stop of the train. The StaStop pushbutton can be used to enable the station-stop feature for that train. The Reverse pushbutton changes the direction of the train's motion if the train is stopped.

\subsubsection{Using the Control Panel}

The best way to familiarize yourself with the features of the Control Panel is to try them, observing their effects as displayed in the Viever window. Below are 
some suggested steps for getting acquainted with the system, using the sample layout of Figure 1.1. ${ }^{3}$

When describing graphics manipulations, we will use the following terms for mouse-oriented input operations. The mouse button to press and release is the left mouse button (for standard workstation window managers).

- To click on a pushbutton or icon, move the mouse pointer into the pushbutton or icon area, then press the mouse button down and release it immediately.

- To drag from one point to another in a window, position the mouse cursor at the starting point, then press the mouse button and hold it down while moving the mouse icon to the finish point. Finally, release the mouse button.

- To drag a slide bar to a value $v$, first position the mouse pointer over the inner region that contains the indicator arrow for that slide bar graphic. Then, press the mouse button and hold it down while moving the mouse cursor right or left until the desired value $v$ is displayed on the numerical display. Finally, release the mouse button. Due to variation in graphics display resolution, it may not be possible to enter arbitrary desired values using a slide bar.

Before starting, identify the controls and indicators for each train and switch block in the Control Panel window. The Speed, Goal and Throttle slide bars for each train display the initial value zero. Each train is operational, and neither of the StaStop and EmerStop indicator lights is illuminated.

Click (once) on the pushbutton for the switch block si 14.

When you click on the pushbutton, the switch block toggles between the straight and turned settings. Observe that there is a delay after pressing the pushbutton before the switch block setting actually changes on the screen. This delay has two causes: communication time and the time that it actually takes for a switch block, which is a large mechanical device, to change setting.

Click on the switch block pushbutton again to toggle the switch block back to the straight setting.

Click on the StaStop and EmerStop pushbuttons for Train 2.

Do not click on the Reverse pushbutton for Train 2 nor any pushbuttons for Train 1 at this time.

\footnotetext{
${ }^{3}$ Suggested actions are enclosed in boxes.
} 
When the StaStop indicator light is on, we say that train is in stationstop mode; likewise, the EmerStop indicator light shows whether the train is in emergency-stop mode. Note that the StaStop indicator light can be illuminated, but the EmerStop indicator light cannot be illuminated yet. An operational train's station-stop mode can be enabled or disabled whether that train is moving or not. Emergency-stop mode cannot be enabled for a train unless that train is moving.

We are now ready to set a train in motion.

Accelerate Train 1 by dragging the Throttle slide bar for that train to a value near $40(\mathrm{~m} / \mathrm{sec})$.

Notice that several things happen when you do this.

- The goal speed indicator, labelled Goal, now shows the throttle value.

- The current speed indicator, labelled Speed, begins changing from the previous speed (zero in this case) towards the goal speed.

- The train at the top of the Viever window begins moving (forward towards the left, in this case).

The goal speed is not reached instantaneously. A train ordinarily changes speed at a fixed rate of acceleration, as explained in Section 2.3.2, and it takes time to accelerate from 0 to $40 \mathrm{~m} / \mathrm{sec}$.

Observe that a block is highlighted in the Viever window if any part of that block is occupied by a train.

Drag the Throttle slide bar to about $55 \mathrm{~m} / \mathrm{sec}$, then drag it to about $45 \mathrm{~m} / \mathrm{sec}$ before the train has completed accelerating to $55 \mathrm{~m} / \mathrm{sec}$.

This exercise shows that a new goal speed value overrides a previous one when the throttle is changed, even if a previous goal speed has not yet been reached.

There is a maximum speed limit of $60 \mathrm{~m} / \mathrm{sec}$ for each block in the sample layout of Figure 1.1. If a train exceeds this limit, that train derails. All minimum speed limits in the sample layout are zero. Using the editor $t$ sed (see Section 1.2 below) it is possible to create new layouts having different shapes and block speed limits or to modify the features of an existing layout. However, there is no provision for changing the attributes of a layout while it is being simulated.

Next, request an emergency stop.

Click on the EmerStop pushbutton for Train 1. 
Observe that the EmerStop indicator light is illuminated while an emergency stop is in progress and goes off as soon as the stop has completed. Another way to stop a train is by simply setting that train's throttle to zero. An emergency stop, like a throttle change, overrides any prior motion plan. Thus, during an emergency stop a train's Goal speed is zero and its current speed decreases toward zero. There are two important differences between using the throttle to decelerate to zero and using emergency stop.

- Emergency stops use a larger deceleration rate.

- Nothing can override an emergency stop, other than derailment or collision of the train.

In particular, dragging the Throttle slide bar has no effect during an emergency stop. Nor does an emergency stop cause the throttle bar to move. The throttle is simply ignored until the emergency stop has completed and the throttle is dragged again.

Station blocks differ from regular blocks in that they have a station-stop feature. To test this feature, perform the following steps.

Click on the StaStop pushbutton of Train 1 so that the StaStop indicator light becomes illuminated (station-stop mode).

Drag that train's Throttle slide bar to $30 \mathrm{~m} / \mathrm{sec}$ or less.

The next time that Train 1 enters a station block (either st 11 or st 16 ), it will immediately begin slowing down so that it comes to a halt exactly at the opposite terminator of that block. Three conditions are required for a train to begin performing a station stop.

- That train must have station-stop mode enabled.

- That train must have speed at most the station-stop speed.

- That train must be entering a station block.

The station-stop speed $(30 \mathrm{~m} / \mathrm{sec}$ in the sample layout of Figure 1.1) is specified for each station block when a layout is created.

Observe that the station-stop indicator automatically goes off as soon as a station stop begins.

Allow Train 1 to complete a station stop.

Unlike emergency stops, it is possible to abort a station stop by changing the throttle value for the train involved. If a station stop is aborted, then no station stop is performed until the three station-stop conditions are again met on entry into a station block.

A train's direction can only be changed when that train is stopped. 
Click on Train 1's Reverse pushbutton (once) while that train is stopped.

Then drag that train's throttle slide bar to a positive value, e.g., $30(\mathrm{~m} / \mathrm{sec})$.

Train 1 will begin to move backwards; that is, the head-end retreats and the tail-end advances.

Up to now, both trains have remained in the Operational state. In order to become acquainted with another train state, try the following.

Let Train 1 travel (backwards) around the layout until it derails at block jn 8 .

When a train enters a join block (such as $j n$ 8) from the tail terminator (the one attached to $\mathbf{I g} 9$ in the sample layout), then the train will derail. If a train derails, the state label for that train changes to Derailed and the train stops moving.

Trains that are not Operational do not respond to commands. Thus, once a train derails, there is no way to set that train in motion again, short of starting another simulation. Page 35 lists all the conditions under which a train will derail.

Click on the pushbutton for switch block sn 14 once so that the switch block changes to the turned setting.

Drag the Throttle slide bar for Train 2 to a positive value, e.g., $30 \mathrm{~m} / \mathrm{sec}$.

Observe that attempting to enter a disconnected terminator of a switch block would cause a train to derail.

Finally, observe what happens when trains collide.

Allow Train 2 to continue until it collides with Train 1.

The state labels of both trains involved in a collision change to Collided. Henceforth, neither train will respond to any commands, so the demonstration has ended-in disaster! Fortunately, simulated trains are inexpensive to replace.

Page 36 lists all possible collision conditions.

\subsubsection{Shutting the Programs Down}

The programs that comprise Trainset may be shut down by selecting Quit All in the Command menu of the Control Panel or Viever. 


\section{Exercises}

1. Start a simulation of the default layout by issuing the ts command. Set both trains in motion around the track simultaneously, with Train 1 travelling forward and Train 2 travelling backward.

2. Perform Exercise 1. Then, begin toggling the setting of the switch block Sw 14 so that Train 1 always passes through $S \nabla 14$ when $S$ w 14 is straight and Train 2 always passes through that switch block when it is turned.

Note: This is not as easy as it may sound! Keeping both trains travelling along different paths in this layout is a challenge, particularly if both are moving as fast as possible. Train speed adjustments and switch block setting changes must be coordinated and must be issued far enough in advance so that the trains neither collide nor occupy a switch block while it changes setting.

\subsection{How to Build a Layout of Blocks and Trains}

tsed is an interactive graphics editor for defining and modifying railroad layouts.

\subsubsection{Invoking tsed}

To start tsed, enter the following command.

$$
\% \text { tsed [flename] }
$$

The editor may also be invoked by entering:

$$
\% \text { ts -edit [filename] }
$$

When tsed is started, two windows appear on your display, as shown in Figure 1.2. The larger window that is overlaid with a grid pattern is the canvas window (Figure 1.2a). A railroad layout can be created in the canvas window. The distance between two adjacent parallel dotted lines in the grid is called a grid division.

The smaller window is called the tools window (Figure 1.2b). It consists of twelve icons representing operations called the tools that are available in tsed for creating and modifying blocks and trains. The tools are applied using the mouse operations described on Page 6. Figure 1.3 shows the tools window together with the names of its tools.

The tools window contains one or more tools for each of the five types of blocks in Trainset. Note that the tools window includes two tools for creating switch blocks. The Switch Block 1 and Switch Block 2 tools differ in the orientation of the switch block; each is a mirror image of the other. Also, there are three tools for creating regular blocks: Straight Block, Arc Block 1 and Arc Block 2. A straight block is a regular block consisting of a line segment, and an arc block is a regular block consisting of a circular segment. Arc Block 1 


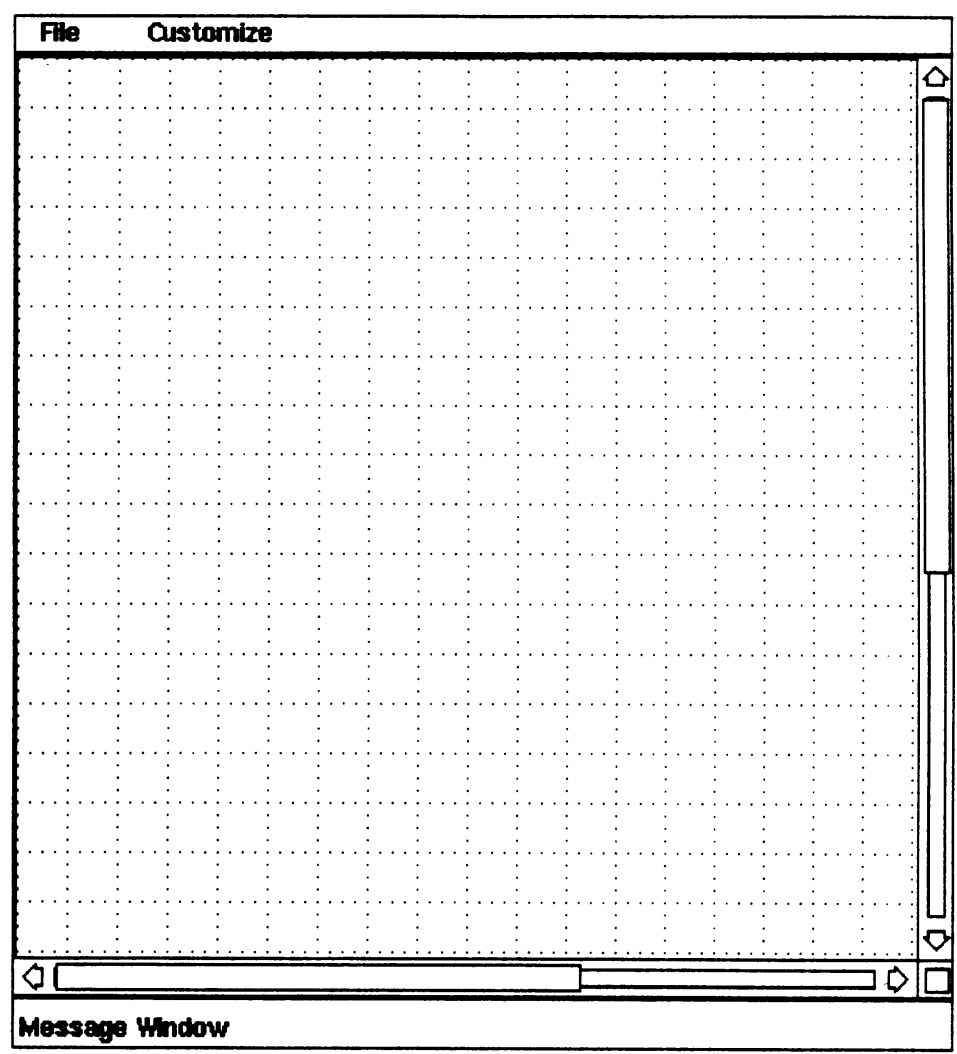

(a) Canvas Window

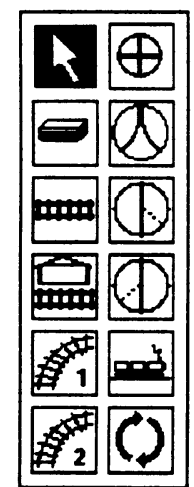

(b) Tools Window

Figure 1.2: tsed Windows

\begin{tabular}{|c|c|c|}
\hline Select & & Cross Block \\
\hline Erase & | & Join Block \\
\hline Straight Block & and & Switch Block 1 \\
\hline Station Block & IIII & Switch Block 2 \\
\hline Arc Block 1 & 1 & Train \\
\hline Arc Block 2 & 2 & Rotate \\
\hline
\end{tabular}

Figure 1.3: Annotated Tools Window 
and Arc Block 2 differ only in the radius of the arcs they create. The Arc Block 1 tool creates arcs with radius two grid divisions, and the Arc Block 2 tool creates arcs with radius four grid divisions.

The remainder of this section is a two-part tutorial on using tsed for creating and editing railroad layouts. Section 1.2.2 introduces you to the various tsed tools. A series of exercises demonstrates how to construct a layout and how to make simple editing changes. Section 1.2.3 explains how to set attributes such as block speed limits, how to save a layout in a file, and how to exit from the editor.

\subsubsection{Using the tsed tools}

\section{The Current Tool}

In tsed, one tool is enabled at any given time; it is called the current tool. The current tool is indicated in the tools window by being highlighted. The message area at the bottom of the canvas window also displays the name of the current tool. On startup, Select is the current tool.

Click on a tool icon other than Select to choose a different current tool. Repeat one or more times.

\section{Regular and Station Blocks}

Straight regular blocks and station blocks are created in a layout by dragging with the mouse from one point to another in the canvas window when the corresponding straight block or station block is the current tool. This procedure creates a line segment between the starting and stopping points of the drag operation. The starting point is called the head terminator of the block, and the stopping point is called the tail terminator.

\section{Click on the Straight Block tool icon.}

Create a horizontal regular block by dragging from right to left starting near the middle of the canvas window, as shown in Figure $1.4 \mathrm{a}$.

All linear blocks (Straight and Station) created by tsed are constrained to be vertical, horizontal or at a $\pm 45^{\circ}$ diagonal. To aid in alignment, tsed enforces additional constraints on the placement and length of a block relative to the dimensions of the canvas window grid.

\section{Click on the Station Block tool icon.}




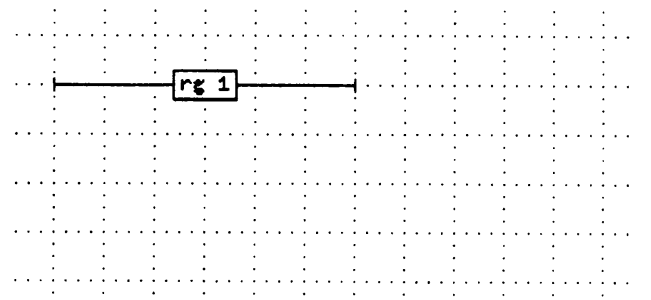

(a)

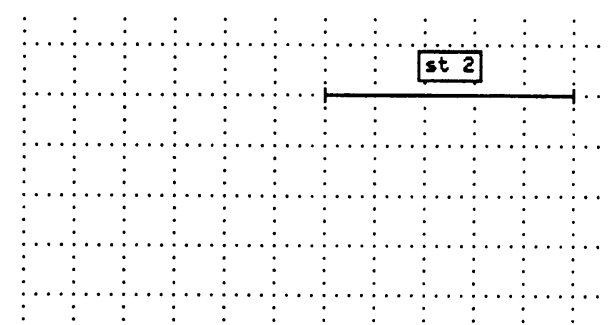

(c)

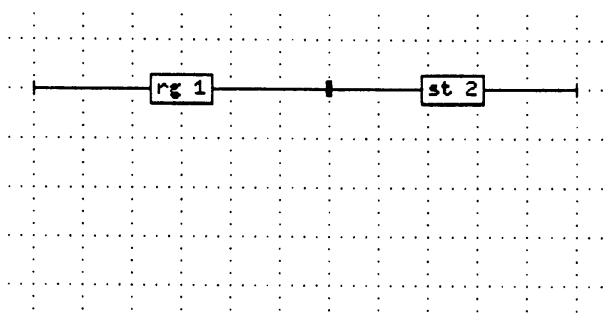

(b)

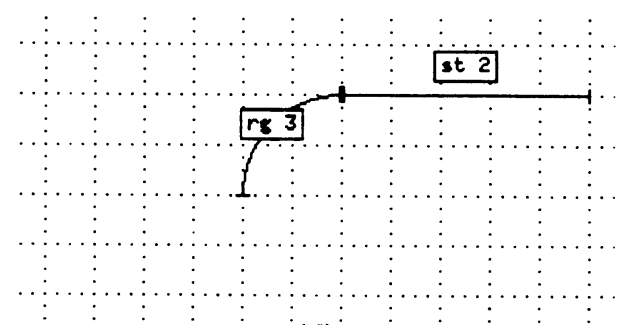

(d)

Figure 1.4: Tutorial Steps

Now create a station block in the canvas window by dragging from the right endpoint of the existing straight block to a point further to the right, as shown in Figure 1.4b.

When a new block is created, tsed establishes a connection with an existing block if the new head terminator is within a few pixels of an existing terminator. tsed also constrains the slope of the new block to match the slope of the existing block at that terminator.

Station blocks and straight regular blocks appear graphically to be identical except for their labels. Regular blocks (whether straight or arc) are labelled rg, and station blocks are labelled st.

The Erase and Select tools are used for modifying objects already on the canvas window. Erase enables you to remove a block or train that you have created. Select enables you to reposition the label for a block or to designate a block whose attributes you wish to change.

Click on the Erase tool icon.

Now, erase the straight block by clicking on it.

Click on the Select tool icon. 
Now drag the label for the station block to a new position above the block.

The result of these changes are shown in Figure 1.4c.

Arc blocks are created by dragging when the current tool is Arc Block 1 or Arc Block 2. The starting point of the drag operation determines the location of the arc block's head terminator. The extent of the arc is determined by the ending point of the drag operation and depends on the location of the head terminator and, if the arc block is attached to another block, the slope of that block.

\section{Click on the Arc Block 1 tool icon.}

Create an arc block connected to the station block by dragging from the left endpoint of the station block toward a point that produces a $90^{\circ}$ arc pointing downward, as shown in Figure 1.4d.

When using the Straight Block, Station Block, Arc Block 1 or Arc Block 2 tools, you can cancel creation of a block after a drag operation has already been initiated by finishing that drag operation within a few pixels of its starting point.

Start dragging to create another arc block connected to the last arc block, then cancel the creation of a new block by finishing that drag operation at its starting point.

\section{Iconic Blocks: Crosses, Joins and Switches}

The Cross Block, Join Block, Switch Block 1 and Switch Block 2 tools always create blocks that have a fixed size and shape. In tsed, these blocks are referred to as iconic blocks. They are created by clicking with the mouse rather than by dragging.

When an iconic-block tool is current and the mouse cursor is in the canvas window, the cursor takes the form of the icon for the current tool. Along the outer circle of such a cursor are enlarged points called hot spots at which connections with other blocks can be made.

Click on the Join Block tool icon. Observe that the mouse cursor changes to the form of a join block whenever the cursor is in the canvas window.

Position the mouse cursor so that the hot spot at its right head is over the unattached terminator of the arc block. This requires slight overlapping between the arc block and the join-block cursor; see Figure 1.5a. 


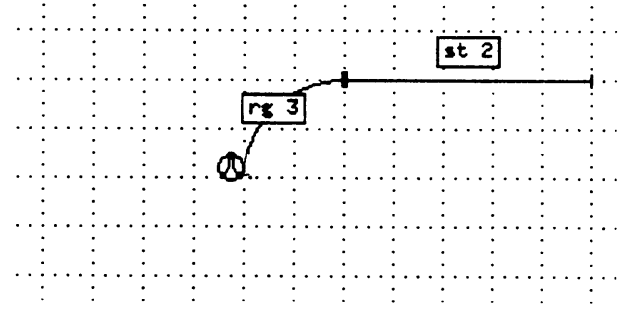

(a) Before Clicking

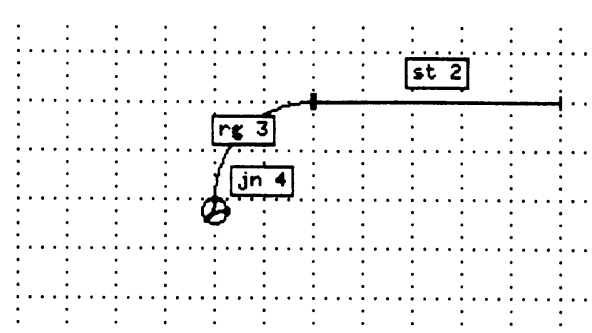

(b) After Clicking

Figure 1.5: Join Block Creation

Now click with the mouse and create the join block of Figure 1.5b.

Note that the newly created join block is constrained so that the slope of the join block and the slope of the arc block agree at their common terminator.

tsed does not make more than one connection when an iconic block is created. Thus, during an editing session, it is probably best to create iconic blocks before creating the regular and station blocks they are attached to.

The Rotate tool enables you to rotate an iconic block clockwise by one hot spot.

\section{Click on the Rotate tool icon.}

Now click on the join block created in step 15. Observe that the join block labelled jn 4 rotates so that its tail terminator becomes the terminator attached to the arc block.

Click on the join block a second time to rotate again.

If an iconic block is not connected to any other block, the Rotate tool rotates it by one-eighth turns. If a switch block is rotated through a full circle using the Rotate tool, it changes orientation.

\section{Trains}

A train may be created in a layout by dragging when Train is the current tool. The drag operation begins at the position desired for the head end of a train, continues along the blocks to be occupied by that train, and stops at the position desired for that train's tail end. The head end of a train is indicated in the layout by an angle bracket, and the tail end is indicated by a square bracket. All blocks occupied by a train are highlighted. The head end of a train is constrained by tsed to start in a regular or station block. 


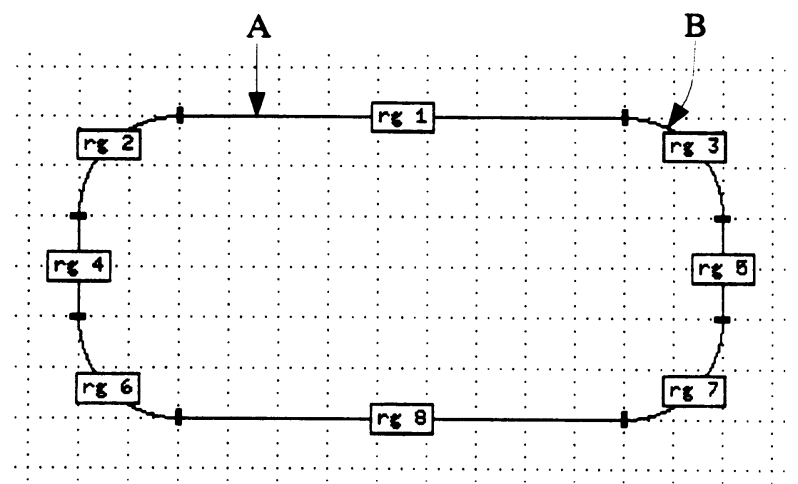

(a) Before Train Creation

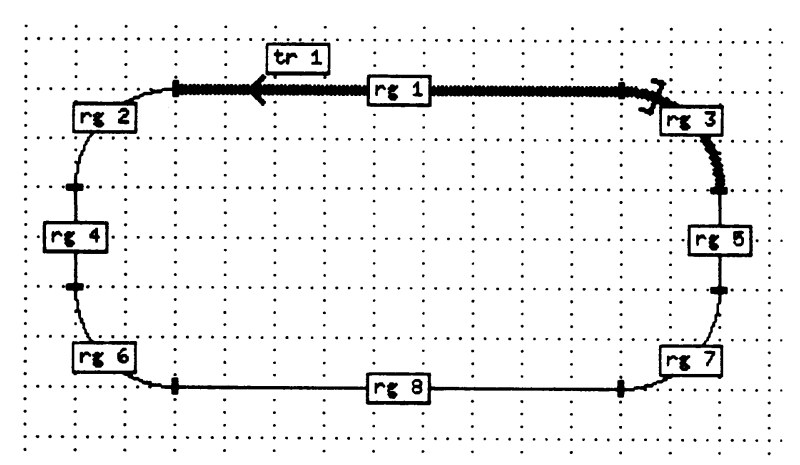

(b) After Train Creation

Figure 1.6: Oval Layout 
Erase all blocks currently on the layout. Then, create an oval layout as in Figure 1.6a made of four straight blocks and four arc blocks, using the Straight Block and Arc Block 1 tools.

It is not necessary that the block identifiers, i.e., the numbers in the labels, match those in Figure 1.6a.

Click on the Train tool icon.

Create a train on the oval by dragging clockwise from the point marked $\mathrm{A}$ to that marked $\mathrm{B}$ in Figure 1.6a, resulting in Figure $1.6 \mathrm{~b}$.

If, while creating a train, you drag across the starting point, the brackets that indicate train ends reverse their directions. Also, observe that a whole block is highlighted if any part of that block is occupied by a train.

You should now feel comfortable with the basic drag and click operations for creating blocks and trains in a layout. In the next part of this tsed tutorial, you will learn how to store a layout in a file, how to specify attributes such as speed limits for individual blocks, and how to exit tsed.

If you would prefer to return to this tutorial at a later time, select quit ${ }^{4}$ from the File menu now, and invoke tsed again when you are ready for the remainder of the tutorial. There is no need to save your present work, since it will not be used in the second part of the tutorial.

\subsubsection{Creating a figure-eight layout file}

Our goal is to create the figure-eight layout illustrated in Figure 1.7 and then save that layout in a file mylayout.1. All blocks in the layout should have minimum speed of $0(\mathrm{~m} / \mathrm{sec})$ and maximum speed of 70 , except that the station blocks at the top and bottom of the layout are to have minimum speed of 0 and maximum speed of 50 .

Select Ier from the File menu. If you are continuing from the first part of the tutorial, tsed will ask if you wish to discard changes in the canvas window; click on the Yes button. In response to the prompt, enter mylayout as the name of the file to be created.

By invoking the Ier command above, mylayout. I becomes the current file name. This name is indicated in the title for the canvas window. Prior to the II .$I$ is automatically appended by tsed if you do not include it. Pathnames that

\footnotetext{
${ }^{4}$ Quit will be discussed in detail on Page 21.
} 


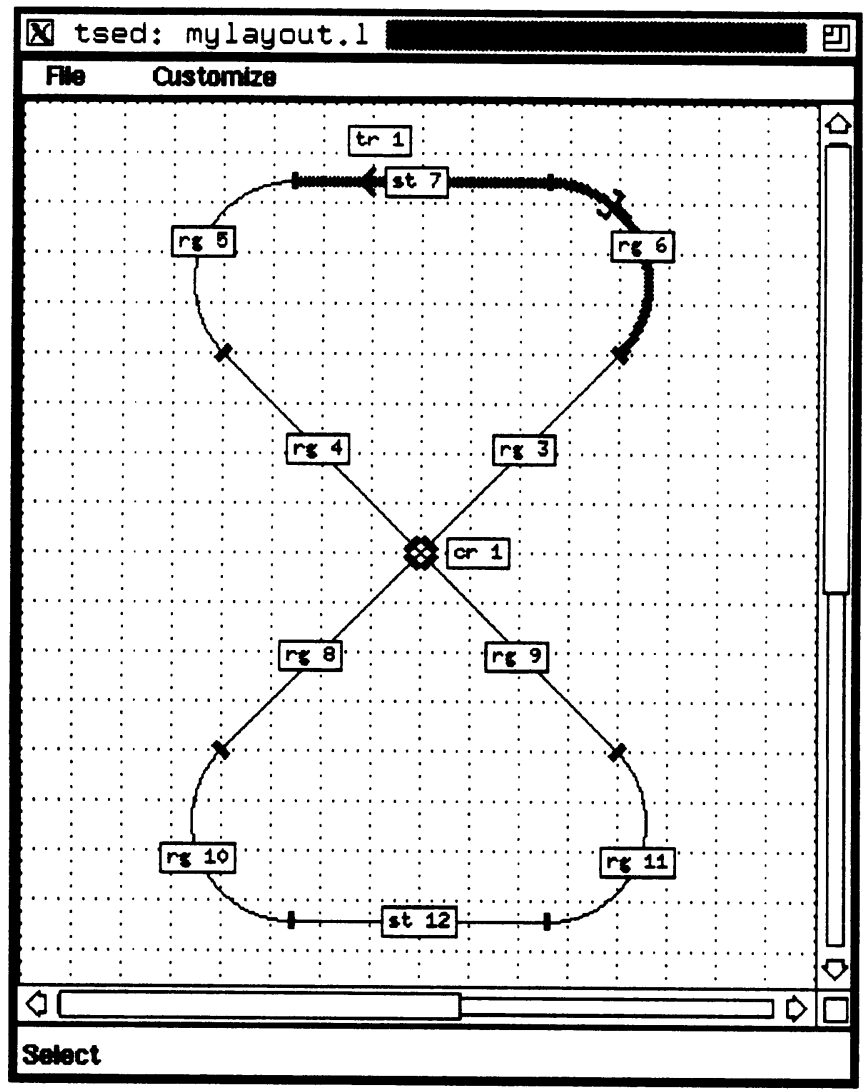

Figure 1.7: A figure-eight layout 


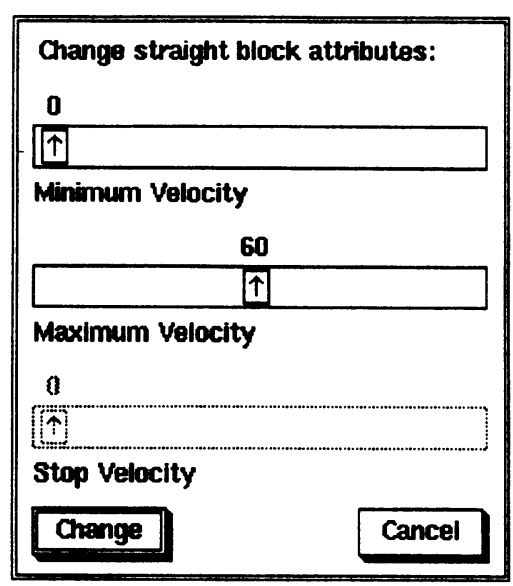

Figure 1.8: Change Default Block Attributes subwindow

do not begin with a slash ' $/$ ' are interpreted relative to the current working directory.

The default minimum and maximum speeds for blocks can be changed by choosing the Change Default Block Attributes entry from the Customize menu. A changed default value applies to blocks created after the change; it does not affect blocks already created. Change Default Block Attributes is itself a menu whose subentries are the various block types.

Change the default attributes for straight blocks by choosing Change Default Block Attributes from the Customize menu and moving the cursor to the right until the subentries appear. Select the Straight subentry.

After this step, a subwindow will appear that contains three slide bars as shown in Figure 1.8. The slide bar on the top determines the minimum speed for straight blocks and is normally 0 . The slide bar in the middle determines the maximum speed, which is 60 at present. The slide bar on the bottom is disabled for straight blocks since it defines the station stop speed, an attribute that is only applicable to station blocks.

Set a new maximum speed by dragging the middle slide bar to 70 . Leave the minimum speed at 0 .

Click on the Change pushbutton to make the new default speed limits effective.

The steps above change the default maximum speed for straight blocks only. We also need arc blocks and cross blocks that have a maximum speed of 70 . 
Change the default maximum speed to 70 for arc blocks, using the Arc subentry in the Change Default Block Attributes menu under Customize and proceeding as above.

Also change the default maximum speed to 70 for cross blocks.

You are now ready to create the blocks of your layout. Refer to Figure 1.7 for illustration of the steps below.

Create a cross block near the center of the layout at an intersection point of two perpendicular dotted lines in the grid.

Rotate the new cross block once using the Rotate tool. The subblocks of the cross should form an ' $x$ ' as opposed to a ' $t$ '.

Create two straight blocks of exactly the same length, each forming the diagonal of a square with sides about 4 grid divisions long, so that the head terminators of the straight blocks are attached to the upper hot spots of the cross block.

Note that the grid assists you in determining when blocks have exactly the same length.

Create two arc blocks with $135^{\circ}$ extent and head terminators at-

tached to the straight blocks that you just created.

Create a station block that connects to both unattached terminators of the arc blocks.

The attributes of an individual block may be customized by selecting that block with the Select tool and choosing Change Block Attributes from the Customize menu.

Click on the Select tool icon.

Click on station block st 7 in the canvas window to select it for customization.

Choose Change Block Attributes from the Customize menu.

In the subwindow that appears, set a new maximum speed by dragging the slide bar in the middle to 50 .

The station stop speed is another attribute that may be adjusted for station blocks using Change Block Attributes. (See Section 2.2.5 for a discussion of the station stop speed.) We will leave the default value at 30 for this layout. 
Create the lower portion of a figure-eight layout using a procedure similar to the previous seven steps.

Place a train on the figure-eight layout.

You have now created the desired figure-eight layout. It is time to save your work and exit from the editor.

Save the figure-eight layout by choosing Save from the File menu.

Either the Save entry or the Save as ... entry in the File menu may be used to save your work in a file. The difference is that Save as ... always prompts you for a file name, while Save uses the current file name if there is one.

Finally, end the editing session:

Exit tsed by choosing Quit from the File menu.

The quit command checks for any unsaved changes before exiting. If any are found, Quit gives you the option of writing them to a file first. The Close command is similar to Quit, except tsed does not exit after checking for unsaved changes. Instead, a Close causes tsed to enter a state with an empty canvas window and no current filename.

\subsection{How to Run a Layout}

A railroad layout mylayout created using tsed can be simulated by issuing the following command.

$$
\% \text { ts -layout mylayout }
$$

A Trainset simulation will start and search for a file called mylayout.1, first searching in the current working directory, then in the standard location for layouts, as explained in the manual page for ts in Appendix D.

\subsection{Control Programs}

The Automatic Control Interface (ACI) is a library of procedures and data structure definitions for writing programs that control a Trainset railroad.

The principal data type associated with the ACI, LayoutData, represents various attributes of the blocks and trains in a railroad layout. LayoutData is described in Section 3.2 and defined in Appendix B.1.

The ACI routines may be classified into five categories. 
1. The ACI GetDownload procedure establishes a network connection with a running railroad simulator and receives a report of the state of the railroad being simulated. GetDownload returns a pointer to an internally allocated data structure of type LayoutData holding the state information that has been received. See Section 3.2 for further details.

2. ACI commands enable a program to change certain attributes of blocks and trains. Seven command types are available.

- SetSwitch initiates a setting change for a switch block.

- Accelerate and Decelerate initiate changing the speed of a train at a constant rate for a specified time period.

- SetSpeed initiates changing the speed of a train towards a specified goal speed.

- SetDirection changes the direction of a train that is stopped.

- EmergencyStop halts a train using a deceleration rate that is quicker than the rate for Decelerate.

- StationStop enables a train to come to a complete stop at a known location in a station block, provided that the train enters that station block slowly enough.

See Section 3.3 for more details about ACI commands.

3. ACI queries enable a control program to obtain state information about a railroad after a download has been received. Four query types are available.

- GetBlockOccupancy indicates whether a specified block is occupied.

- GetSwitchPosit returns the setting of a switch block.

- GetTrainStatus indicates whether a train is operational.

- GetTrainyotion indicates whether a train is moving.

See Section 3.4 for more information.

4. ACI voting service procedures, Set Seq Iumber and HerSeqIumber, interact with a command arbitration facility in the simulator. This service is useful when implementing fault-tolerant control programs. See Section 3.5.

5. ACI utility procedures, InitTimer, GetTimer, AwaitTimer, CancelTimer and Sleep, provide high-level general purpose timing facilities. See Section 3.6. 


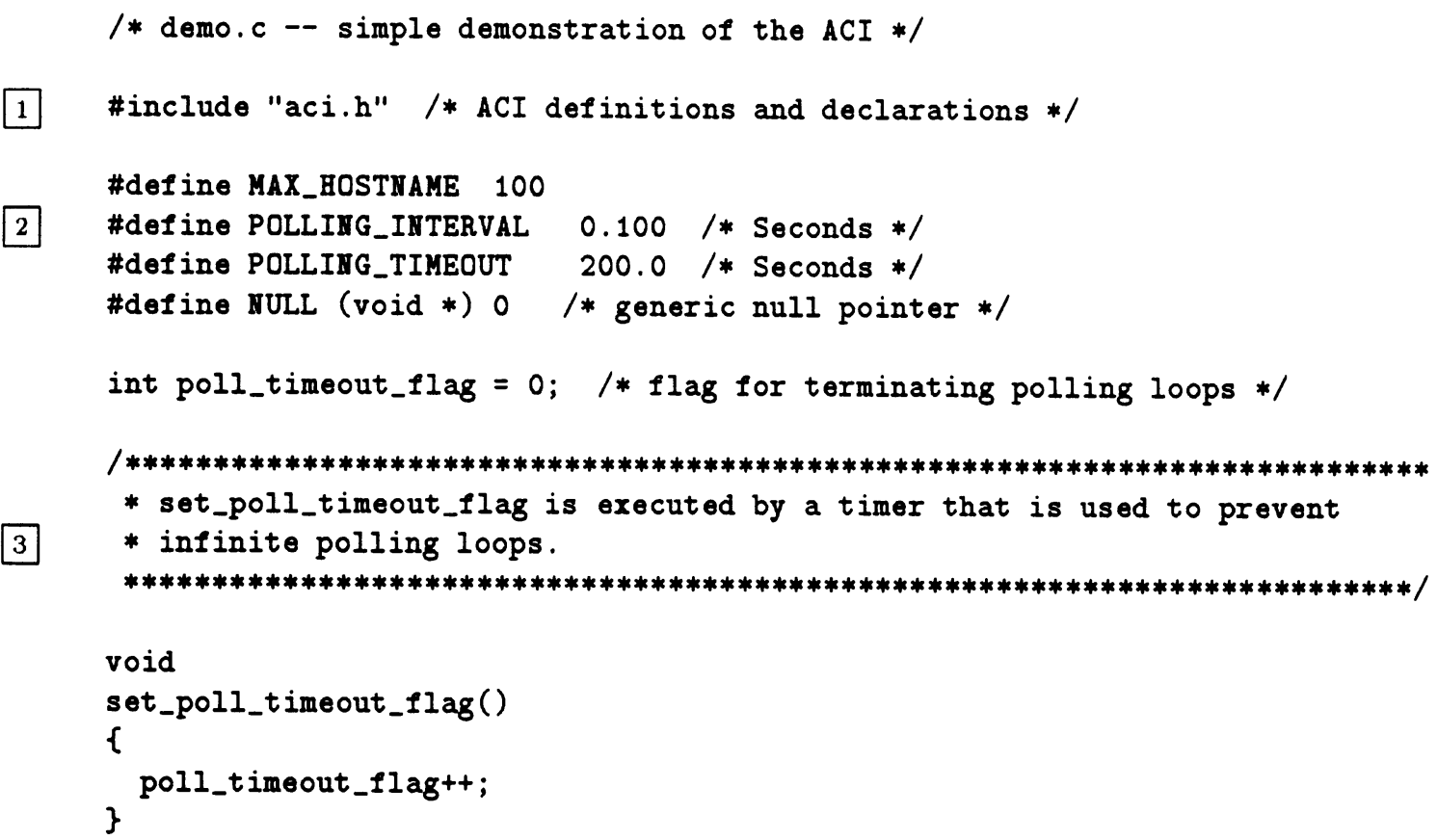

Figure 1.9: demo.c, an example using the ACI (beginning). 


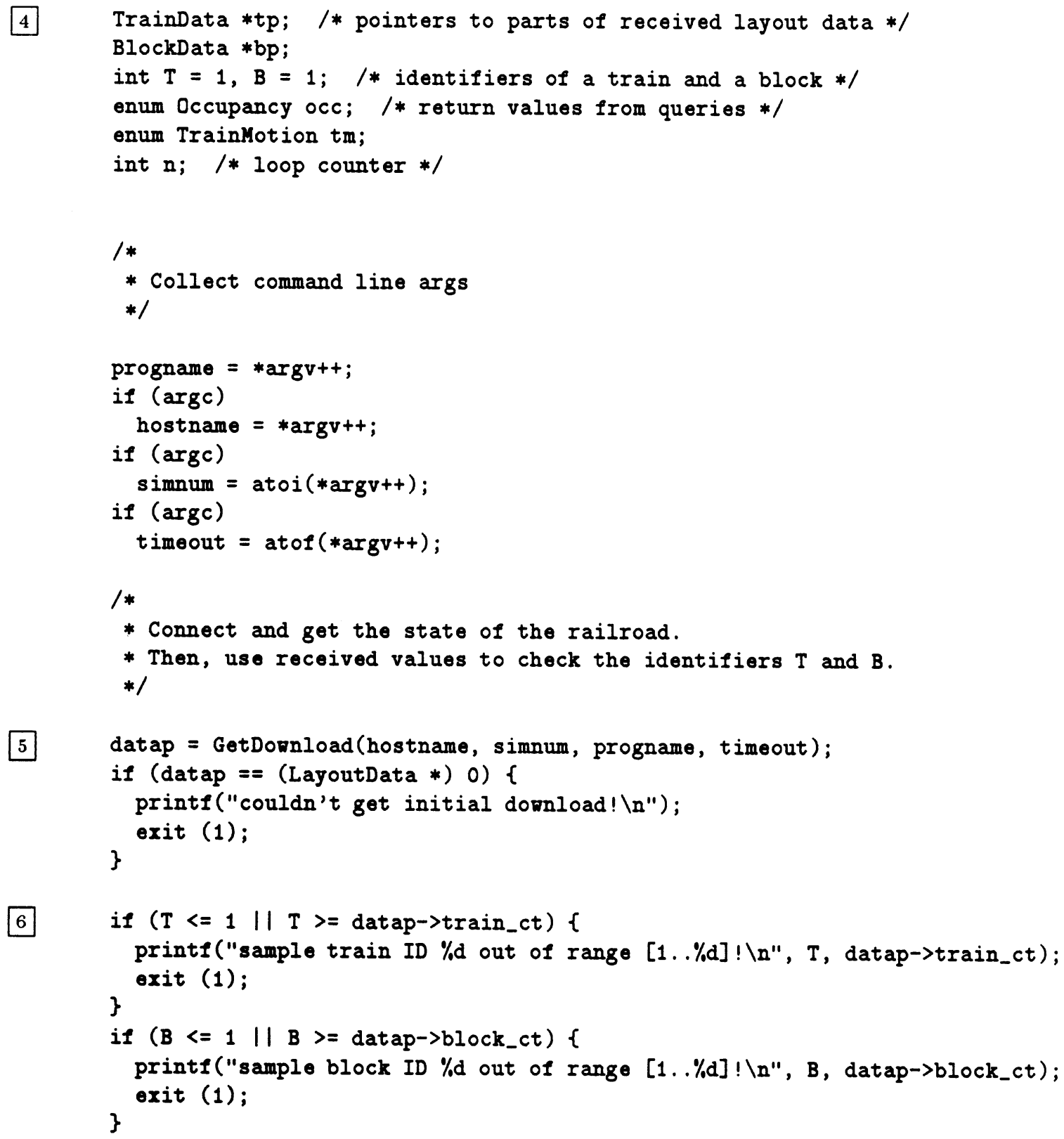

Figure 1.9: demo.c, an example using the ACI (continued). 


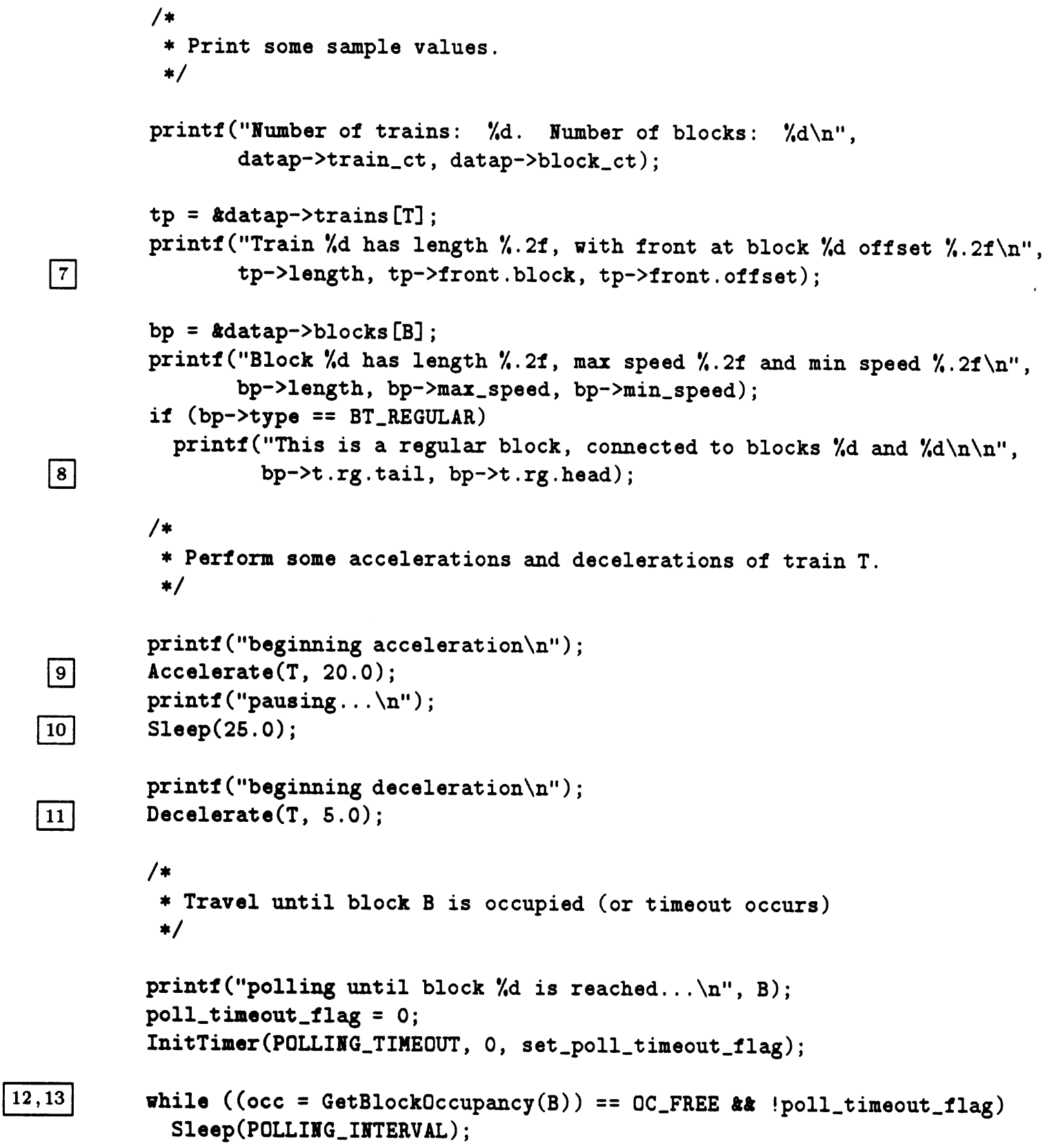

Figure 1.9: demo.c, an example using the ACI (continued). 


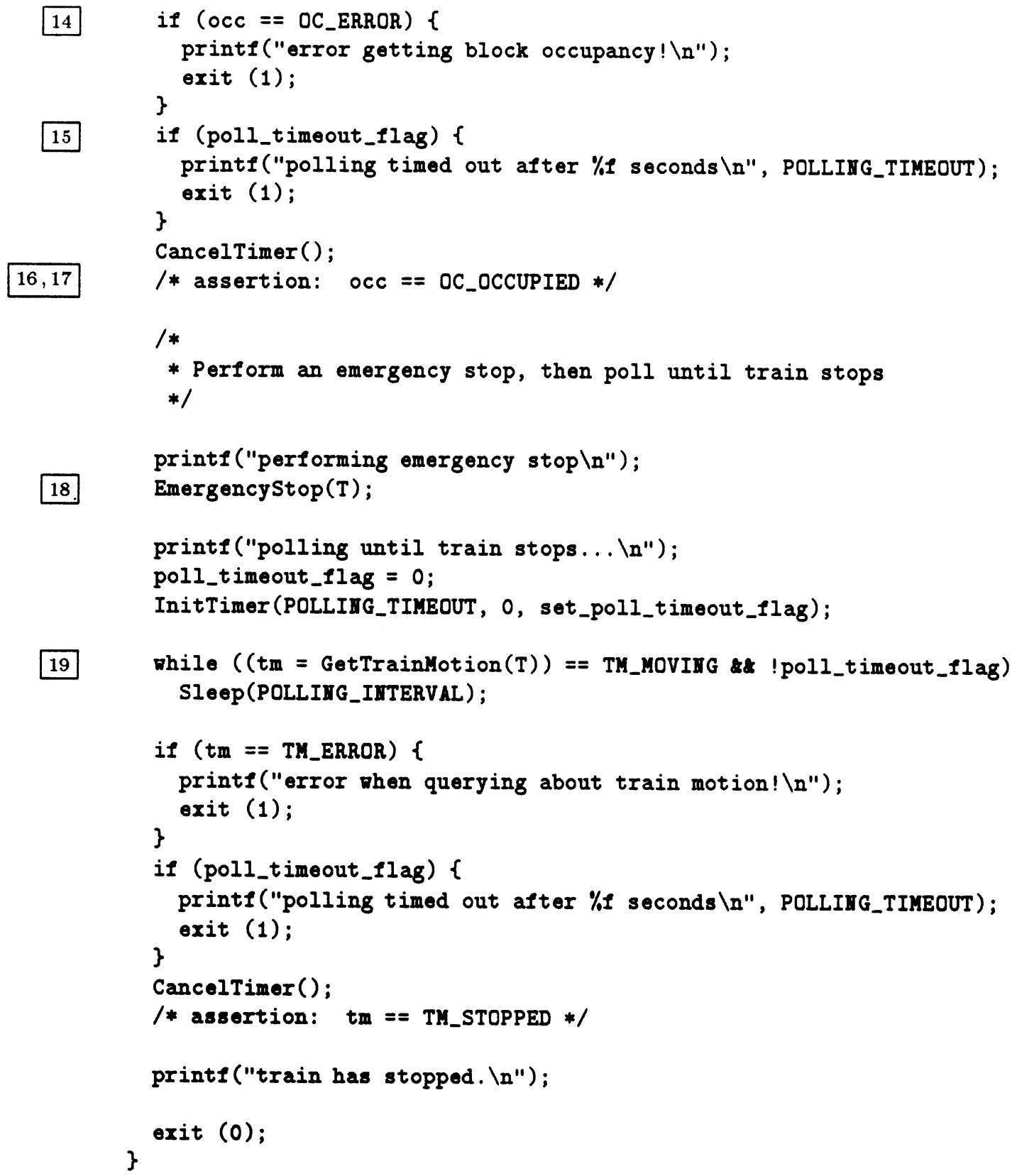

Figure 1.9: demo.c, an example using the ACI (concluded). 
The example program demo.c (see Figure 1.9) illustrates the use of the ACI. This program connects to a simulation and receives the state of a railroad, then attempts to move a train in that railroad to a certain block and perform an emergency stop. Some key points about the code are indicated by numbers 1 , 2 , etc.

1 The include file aci.h contains declarations and definitions required for compiling a source file that uses the ACI. In order to create an executable, one must link with the ACI library libaci.a.

2 Time values passed to the ACI timer procedures are always floating-point quantities representing seconds. An ACI type Seconds is defined for such quantities.

3 set_polling_timeout is the procedure that will be invoked if a tsuser timer expires. ${ }^{5}$ Such a procedure cannot be invoked with arguments, so it changes a global variable polling_timeout in order to inform the main program about timer expiration.

4 The file aci.h defines a number of data types besides LayoutData. The type TrainData is used to represent download information received for a train; likewise, BlockData represents download information for a block. Both TrainData and BlockData are component types used in the definition of LayoutData. Enumerated types, including Occupancy and TrainMotion, are used for command arguments and query return values. Trains and blocks have unique positive integer identifiers.

5 GetDownload takes four arguments, returns a null pointer on failure, etc. Chapter 3 is the reference for this and the other ACI procedures.

$6,7,8$ Several examples of references to a LayoutData structure follow the invocation of GotDownload. More direct references such as

$$
\begin{aligned}
& \text { datap->trains [T-1] . length } \\
& \text { datap->blocks }[B-1] \text {.t.rg.tail }
\end{aligned}
$$

could be used in place of those that involve tp and bp in demo.c. Observe that the index of a train in datap->trains is always one less than that train's identifier. A similar remark holds for blocks.

The function PrintDownload in Appendix B.2 includes examples of references to every component in a LayoutData structure.

Checking the values of $T$ and $B$ at 6 is not strictly necessary in this program, since their values are known to be valid in this case. It is a good defensive programming practice to include such checks anyway, as protection against future changes.

\footnotetext{
${ }^{5}$ Note: As explained in Section 3.6, a call to InitTimer overrides any prior calls. Thus, we speak of "the ACI timer," because only one such timer can be in effect at any time.
} 
9 ACI commands such as Accelerate are non-blocking and return no values. They print no warnings about unreasonable arguments. The command

Accelerate(T, 20.0);

requests that train $\mathrm{T}$ accelerate for a duration of 20 seconds, increasing its speed during that period at the predefined fixed acceleration.

10,11 The instruction

$$
\text { Sleep (25.0); }
$$

causes demo.c (not the Trainset simulator!) to suspend execution for 25 sec. This is long enough that the subsequent Decelerate command 11 is unlikely to interfere with the prior Accelerate command 9 .

The train would begin to reduce its speed as soon as the Decelerate command is received, even if the prior acceleration had not been in effect for the acceleration's entire duration. For example, if the sleep had been for 10 seconds instead of 25 , then train $\mathrm{T}$ would accelerate for about 10 seconds, then decelerate for 5 seconds.

12 The query GetBlock0ccupancy is used so that train $T$ may reach block B. That query is issued frequently until block B is found to be occupied or until an error or timeout occurs. This technique of frequent querying is called polling. The ACI timer is set up before the while statement to provide timeout mechanism for leaving that loop.

13 The loop guard condition shows that there are two ways to leave the loop.

- If occ differs from OC_FREE, i.e., occ has value OC_OCCUPIED or OC_ERROR, then the loop will be exited. (Query errors can be caused by invalid arguments, loss of communication with the railroad, etc.)

- If polling_timeout has a non-zero value, then the loop will be exited. polling_timeout changes from zero to a non-zero value if the timer expires (see 3 and the invocation of InitTimer).

$14,15,16$ Before concluding that occ $=$ OC_OCCUPIED, which would indicate occupancy of block $B$, it is necessary to eliminate the other events that can cause the loop to exit.

17 A period of time elapses between the moment that block B becomes occupied and the time when the control program demo.c can act on that information. This time period arises from the following causes.

- Delay from polling. Some time necessarily elapses between the moment that block B becomes occupied and the time when that sensor is checked. The sensor is checked by each successful call to the 
query GetBlockOccupancy. Thus, if GetBlockOccupancy succeeds this period is bounded by the execution time of one iteration of the polling loop, except possibly when block $B$ is found to be occupied on the first GetBlockOccupancy query.

- Network delay. This is the time required for a sensor value to be communicated to the control program.

- Local processing delay. Once the process that is running demo.c receives a communication that block $B$ was occupied, several further steps occur: the query function returns; the polling loop exits; checks are performed in order to deduce that block occupancy was in fact the reason for loop exit; and the timer is cancelled. Each of these steps requires local processing time.

Such delays can affect the correctness of control programs. For example, the occupancy of a block might change by the time that a control program could take action on that occupancy information. In particular, it is not correct to conclude that block B is currently occupied based solely on the fact that occ has the value OC_OCCUPIED at 17 .

18 The command EmergencyStop causes a train to reduce its speed to zero quickly. Unlike Accelerate and Decelerate, the effects of EmergencyStop cannot be interrupted by another command.

19 Polling with the query GetTrainMotion is used in demo.c to determine when the train has come to a complete stop.

\section{Exercises}

1. Write a program that uses the ACI to cause both trains in the sample layout (see Figure 1.1) to move around the track for five minutes, then stop. (Compare to Exercise 1 of Section 1.1.2.) The trains need not travel on different loops.

2. As in the loop 12, a timer is set up just before the while statement 19 in order to guard against an infinite loop. Is this timer necessary, or is that loop certain to exit in a reasonable amount of time without a timer? (Hint: Consider the specifications of GetTrainyotion in Section 3.4.4.) 


\section{Chapter 2}

\section{Trainset Railroads}

\section{$2.1 \quad$ Introduction}

Trainset railroads are idealized versions of real railroads. This chapter discusses the attributes and operation of Trainset railroads. You will see that while Trainset railroads are simpler than their real-life counterparts, the simplifications are ones that do not make it appreciably easier to write programs to control the railroad.

\subsection{Blocks}

In a Trainset railroad, a layout consists of an assembly of blocks together with movable trains that occupy some of those blocks. See Figure 1.1 for an example.

Every block is assigned a unique block $I D B$, a length $L_{B}$, a maximum speed limit $M X_{B}$ and a minimum speed limit $M N_{B}$. We expect ${ }^{1}$

$$
0 \leq M N_{B} \leq M X_{B} \leq M A X F L O A T \text {. }
$$

Each block has a set of terminators that delimit the track implementing that block.

As in real railroads, each block has an associated sensor called a track circuit, that indicates whether that block is occupied by a train. Polling a track circuit only returns one bit of information signifying whether the associated track block is occupied. Note that it is not possible to learn the exact location of a moving train from the information returned by a track circuit.

A block may have between two and four terminators, depending on its type. There are five types of train blocks: regular, join, switch, cross and station blocks. These are illustrated in Figures 2.1-2.5.

\footnotetext{
${ }^{1} M A X F L O A T$ is the largest floating point number on the computer system. The standard $m k s$ units of measure are used throughout this document.
} 


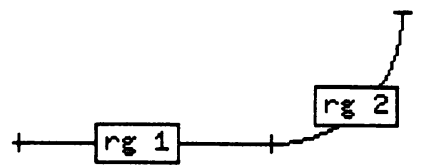

Figure 2.1: Regular Blocks

Two blocks are attached to each other if each of the blocks has a terminator that is associated with the other block. Trainset block layouts are subject to the following restrictions.

- A terminator may be associated with at most one block.

- A block may not be attached to itself.

- Blocks may not be attached to each other more than once, e.g., circular track configurations that consist only of two half-circle blocks are prohibited.

These restrictions make it simpler to describe attachments between blocks, but do not limit the topology of Trainset layouts. For example, we could easily construct a circular track configuration using two quarter circles and a semicircle.

It is not necessary that all terminators of a block be attached to other blocks. Of course, a train will derail if it attempts to exit from a block at an unattached terminator.

\subsubsection{Regular Blocks}

Each regular block has two terminators. Such a block can have the shape of a line segment or a circular arc. See Figure 2.1.

\subsubsection{Join Blocks}

A join block has three terminators and allows two train routes to merge. Two of the terminators, called the heads, are each connected to the third terminator, called the tail.

A train can occupy the track between either of the heads and the tail. Trains can enter a join block at either head and exit through the tail. A train derails when it either enters a join block at the tail or leaves from one of the heads. 


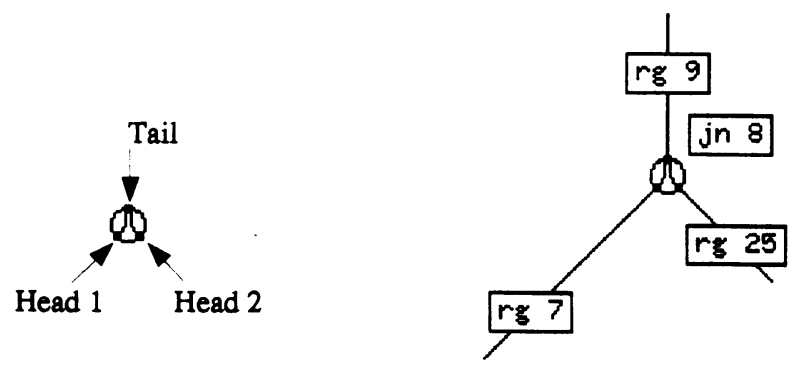

Figure 2.2: Join Block
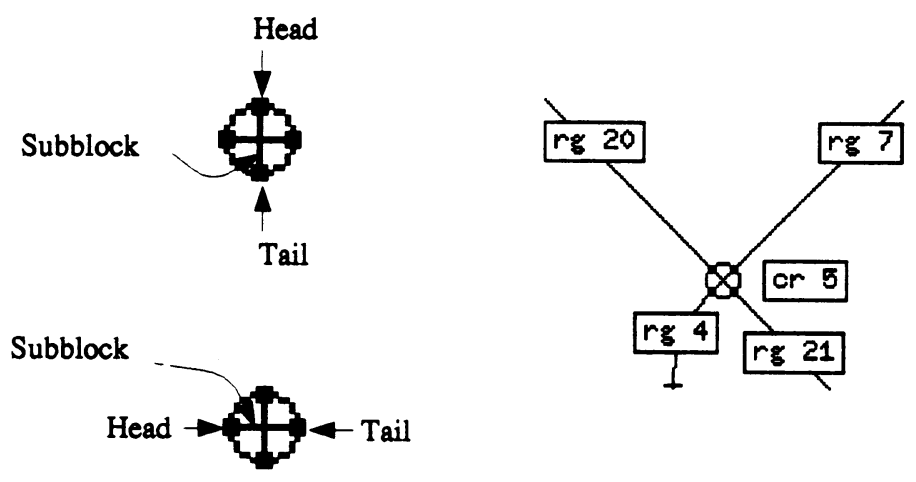

Figure 2.3: Cross Block

Join blocks are assumed to be $L E N_{\text {join }}$ meters long measured from the tail to either head terminator. ${ }^{2}$ At most one train may occupy a join block at any time. If a second train enters a join block that is already occupied, then a collision occurs.

\subsubsection{Cross Blocks}

A cross block (see Figure 2.3) allows a track to intersect itself, so that "figure eights" and related designs may be built. A cross has four terminators, and a train may only travel between any opposing pair-turns are not allowed. Thus, cross blocks behave like two short regular blocks, called subblocks, with the

\footnotetext{
${ }^{2}$ Constants such as $L E N_{\text {join }}$ are determined at the time of installation. See the installation manual for details.
} 

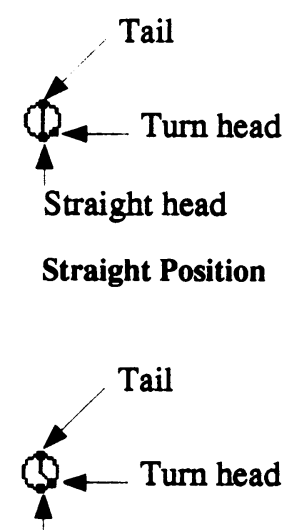

Straight head

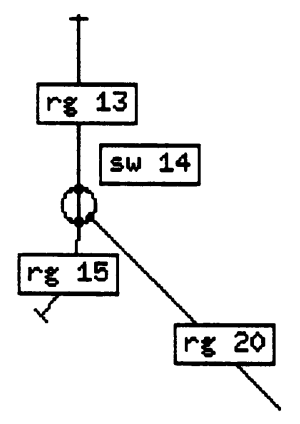

Turn Position

Figure 2.4: Switch Block

same length and speed limits, that have been overlaid at their midpoints. A cross block and both of its subblocks are occupied whenever either of those subblocks is occupied. Cross subblocks are $L E N_{\text {cross }}$ meters long. A collision occurs when a cross block becomes occupied by two or more trains.

In order to distinguish between the subblocks of a cross block, each has its own block ID. One of these also serves as the cross block's ID.

\subsubsection{Switch Blocks}

A switch block (see Figure 2.4) has three terminators, labelled tail, straight head and turn head, and consists of a movable track segment that has two settings and a mechanism to move this track between those settings. Depending on the setting of the movable track, either the train may travel between the tail and straight head (in either direction) or between the tail and turn head. Thus, a switch block can be part of two different train routes, according to that switch block's setting.

A switching time $T_{\text {switch }}$ must elapse for a switch block to change from one setting to the other. The switch setting is undefined during this time period. If a switch is commanded to change to one setting (e.g., straight) while it is already in that setting or in the process of changing to that setting, then that setting-change command has no effect. If the setting-change command was to switch to the other setting, then the switch begins changing to the new setting. It takes a full $T_{\text {switch }}$ seconds to change to a new setting, even if the switch 


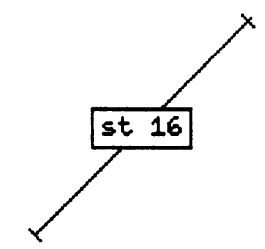

Figure 2.5: Station Block

setting was undefined at the time of the setting change command.

A train attempting to traverse a switch block will derail under the following circumstances.

- The switch block has undefined setting.

- A switch change is attempted on the switch block.

- The train enters the turn head terminator of a straight switch, or the straight head terminator of a turned switch.

Switch blocks are $L E N_{\text {switch }}$ meters long measured from the tail to either of the other terminators. At most one train may occupy a switch block at a time or a collision will occur.

A switch block that is occupied by a derailed or collided train will not respond to setting change commands.

\subsubsection{Station Blocks}

Station blocks are like regular blocks (see Figure 2.1) except that they allow a train to stop at a fixed specified location. Using a station block is the only way to be sure of the exact position of a train after it has begun moving, because track circuits only reveal whether a block was occupied sometime in the past.

Each station block $B$ has a station-stop-speed $V S S_{B}$. A station stop begins whenever a train that is in station-stop mode (see Section 2.3) enters a station block while travelling with speed at most $V S S_{B}$. Under these conditions, the train will automatically begin to slow down so that it will come to a complete stop exactly at the opposite terminator of the station block.

A station stop by a train can be aborted by commanding that train to change velocity or acceleration. 


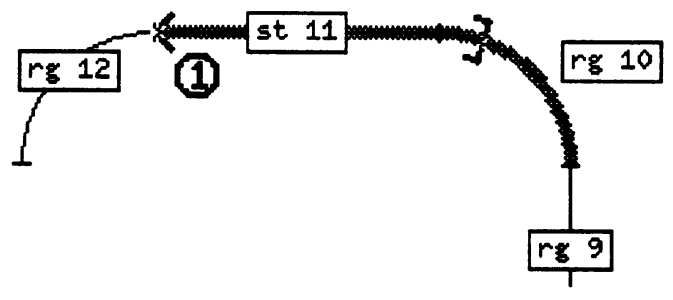

Figure 2.6: Block occupancy.

\subsection{Trains}

Every train is assigned a unique train $I D T$ and a length and has the following attributes that may change during a simulation.

- A speed $v_{T}$ and an acceleration $a_{T}$.

- Two ends, labelled head and tail, that are points within blocks.

- A direction dir ${ }_{T}$.

- An emergency-stop mode $e s_{T}$ and a station-stop mode $s s_{T}$, which may be enabled or disabled.

We expect $0 \leq v_{T} \leq M A X F L O A T$. The distance along the track between a train's ends is always that train's length $L_{T}$.

Each train in a layout always occupies a sequence of attached blocks. A train occupies a block if the interiors of that train and that block intersect. For example, suppose that the train in Figure 2.6 has just completed a station stop, so that one end of that train is at a terminator. That train occupies blocks Ig 10 and st 11 , but does not occupy block rg 12 . If that train moves slightly to the left (i.e., forward), it will then occupy block Ig 12.

A train's direction $\operatorname{dir}_{T}$ determines which of the two ends advances if that train moves forward. The train end that advances is called the front, and the other end is called the rear. For example, suppose that the head of a train is the front. If that train's direction is changed, then the train's tail will become the front and the head will become the rear. A train's direction can only change when that train is not moving, that is, when $v_{T}=a_{T}=0$.

\subsubsection{Train States}

A train can be in one of three states: operational, derailed or collided. Figure 2.7 shows possible transitions between these states.

A train's state changes from operational to derailed under any of the following conditions: 


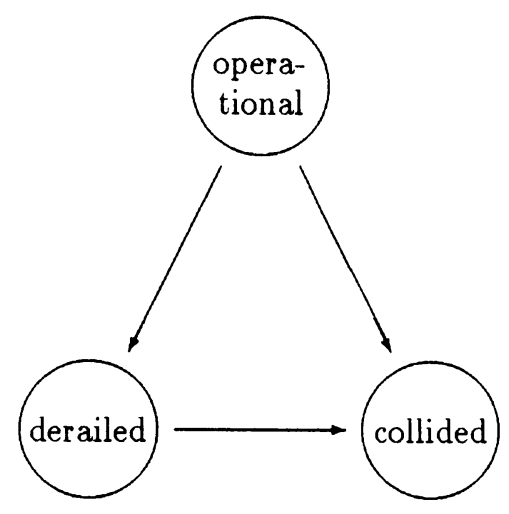

Figure 2.7: Train state transitions.

- That train occupies a block and the train's speed $v_{T}$ violates one of that block's speed limits, $v_{T}<M N_{B}$ or $M X_{B}<v_{T}$.

- The front of that train exits a block at an unattached terminator.

- The front of that train enters a join block at the tail terminator or exits a join block from a head terminator.

- That train occupies a switch block that has undefined setting.

- A switch change is attempted on a switch block occupied by that train.

- That train enters the turn head terminator of a switch in the straight setting or the straight head terminator of a switch in the turned setting.

A train's state changes to collided if there is another train in the layout such that either of the following conditions holds.

- Both trains occupy the same switch, join or cross block.

- Both trains occupy the same block at the same point.

\subsubsection{Laws of Motion}

In Trainset, the acceleration of a train is one of the constant values $A C C, 0$, $-A C C$ and $-A_{\text {emer }}$, except during a station stop. We expect

$$
0<A C C<A_{\text {emer }} \leq M A X F L O A T .
$$

If the acceleration $a_{T}$ of a train is constant during a time interval $\left[t_{0}, t_{1}\right]$ and $v\left(t_{i}\right)$ is the speed $v_{T}$ of that train at time $t_{i}$, then

$$
v\left(t_{1}\right)=v\left(t_{0}\right)+a_{T} \cdot\left(t_{1}-t_{0}\right)
$$


For such a train, if $x\left(t_{i}\right)$ represents the position of an end of that train at time $t_{i}$, then

$$
x\left(t_{1}\right)=x\left(t_{0}\right)+v\left(t_{0}\right) \cdot\left(t_{1}-t_{0}\right)+\frac{a_{T}}{2} \cdot\left(t_{1}-t_{0}\right)^{2} .
$$

A train's station-stop mode turns on the station-stop feature for that train. A station stop begins if the station-stop mode of a train is enabled and that train enters a station block with speed $v_{\text {. }}$ satisfying

$$
0 \leq v_{T} \leq V S S_{B},
$$

where $V S S_{B}$ is the station-stop speed of that block. The station-stop mode of that train is disabled as soon as a station stop begins.

A station stop by a train can be aborted by setting that train's speed or acceleration during that station stop. If a train performs a station stop in a station block and that station stop is not aborted, then all of the following will hold upon its completion.

- The train's speed $v_{T}$ and acceleration $a_{T}$ will be 0 .

- The train's front will be at the opposite terminator of that station block.

- The train will occupy that station block.

A station stop's completion may be observed using the query GetTrainMotion discussed in Section 3.4.

The emergency-stop feature allows a train to stop at a faster rate than usual. A train performs an emergency stop when its emergency-stop mode is enabled. During an emergency stop by a train $T$, its acceleration satisfies $a_{T}=-A_{\text {emer }}$. The emergency stop finishes as soon as $v_{T}$ becomes 0 . At that time, the emergency-stop mode for $T$ is disabled and its acceleration $a_{T}$ becomes 0.

A train does not respond to commands during an emergency stop. Thus, emergency stops cannot be aborted. 


\section{Chapter 3}

\section{Automatic Control Interface (ACI)}

\subsection{Introduction}

The Control Program Interface (CPI) is used for writing computer programs that interact with Trainset railroads. It consists of two layers, the Automated Control Interface (ACI) and the Low-Level Interface (LLI), as illustrated in Figure 3.1.

The ACI layer consists of library routines that can be used in a control program to establish communication with a simulation of a Trainset railroad, obtain the state of that railroad, interact with the railroad's layout using commands and queries and use the Trainset voting service. ${ }^{1}$ The ACI also provides some local timer utilities. This layer is intended to meet the needs of most control program writers.

\footnotetext{
${ }^{1}$ The voting service facilitates writing fault-tolerant control programs. See Section 3.5.
}

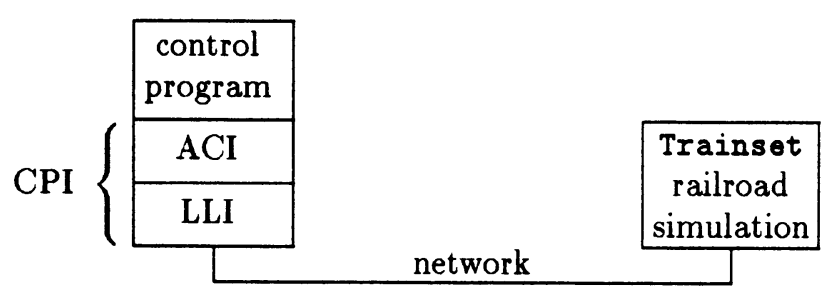

Figure 3.1: Communication interface layers. 
The LLI layer has most of the same capabilities as the ACI, but at a more primitive level that requires a programmer to manage communication and message buffers directly.

This chapter describes the ACI layer. Usage details are described in a reference format using $\mathrm{C}$ language syntax. Chapter 4 presents the LLI layer. For programming examples that use the ACI layer, see Section 1.4 and Appendix B.2.

A Trainset railroad can accept three kinds of messages from CPI clients. A state download request asks for a railroad's state. A command can affect a railroad layout, e.g., by changing a switch block's setting or setting a train's acceleration. A query requests information about a block or train.

Trainset railroads silently ignore messages that contain syntax errors.

\subsection{Initial-State Download}

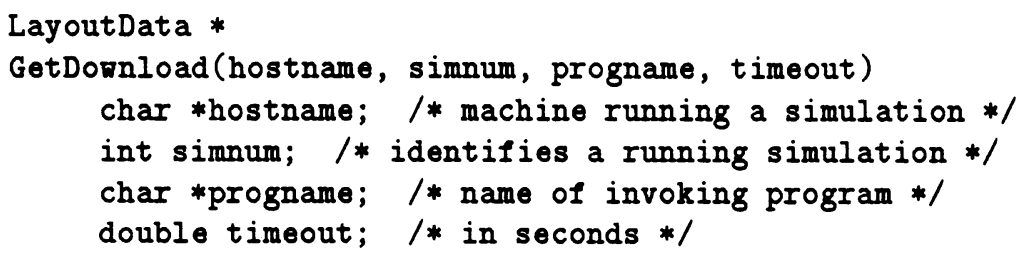

Executing GetDownload causes a network connection to be established with a railroad simulation and information about the state of the railroad to be received from that simulation. The download is stored in an internal static data structure of type LayoutData defined in Appendix B.1.

hostname and simnum ${ }^{2}$ must identify an executing Trainset railroad simulation, and timeout must be positive, or GetDownload will fail. If hostname is an empty string, then the local host is used.

The address of the LayoutData structure is returned if execution succeeds. A message is printed and a NULL pointer is returned on failure. Possible failure conditions are:

- timeout is not a positive floating-point value.

- A network connection could not be established before timeout seconds elapsed.

- There is no simulation on the host hostname with identifier simnum.

\footnotetext{
${ }^{2}$ simnum determines which well-known ports are to be used when initializing communication with a railroad simulation. Multiple railroad simulations may be run simultaneously on the same host if they use different simnum values.
} 
- An error was detected while parsing the download.

- GetDownload has already been called successfully by this client at a prior time.

The download consists of the following.

General parameters:

- The number of blocks $N_{\text {blocks }}$ and of trains $N_{\text {trains }}$ in the layout. Block IDs range from 1 to $N_{\text {blocks }}$ and train IDs range from 1 to $N_{\text {trains }}$.

- The standard acceleration rate $A C C$ and emergency-stop acceleration rate $A_{\text {emer }}$ for trains.

- The switching time $T_{\text {switch }}$ for switch blocks.

- The voting window $W_{\text {voting }}$ and voting quorum $Q_{\text {voting }}$ for commands to be accepted. See Section 3.5.

For each block:

- The ID $B$, type and length of the block.

- The maximum speed limit $M X_{B}$ and minimum speed limit $M N_{B}$.

- The attachments for that block. A block ID is specified for each attached terminator and 0 for each unattached terminator.

- For station blocks, the station-stop speed $V S S_{B}$.

- For subblocks of cross blocks, the block ID of the cross block.

For each train:

- The ID $T$ and length of that train.

- The locations of that train's head and tail ends. The location of an end of a train is specified by giving the ID of a block containing that end and the offset of that end within the block. Offsets are measured from the tail terminator of a block.

\subsection{Commands}

A CPI client can cause a change in a Trainset railroad by issuing a command. Such a change is called a layout action. Only trains and switch blocks can be affected by commands. 
Each command gives rise to at most one layout action. A command fails to cause a layout action if the preconditions of that command are not satisfied. For example, a SetDirection command causes a new direction to be assigned to a train only if that train is operational and not moving. Also, multiple commands may optionally be required to cause a single layout action, as described in Section 3.5.

There are seven types of command that a CPI client can send to a Trainset railroad: SetSwitch, Accelerate, Decelerate, SetSpeed, SetDirection, EmergencyStop and StationStop. These are specified as follows.

\subsubsection{Set Switch}

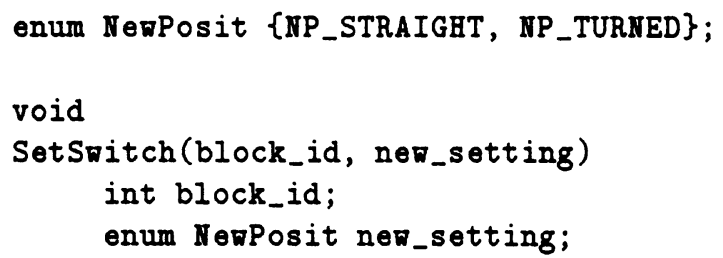

SetSwitch causes the switch block identified by block_id to begin changing setting to new_setting. A setting change requires $T_{\text {switch }}$ seconds to complete.

block_id must identify an unoccupied switch block that is neither in the setting new_setting nor in the process of changing to that setting; otherwise no action is taken.

\subsubsection{Accelerate and Decelerate}

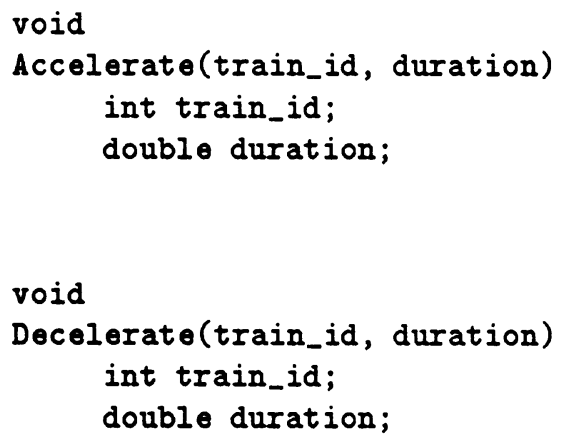

Accelerate and Decelerate cause the acceleration $a_{\text {train_id }}$ of the train identified by train_id to be assigned the following value.

$$
a_{\text {train_id }}= \begin{cases}+A C C & \text { for Accelerate } \\ -A C C & \text { for Decelerate }\end{cases}
$$


$a_{\text {train_id }}$ is assigned the value 0 after either duration seconds elapse or the train's speed reaches 0 , whichever comes first, unless $a_{\text {train_id }}$ is changed again or the train collides or derails.

duration must be non-negative, and train_id must identify an operational train that is not performing an emergency stop; otherwise no action is taken.

\subsubsection{Set Speed}

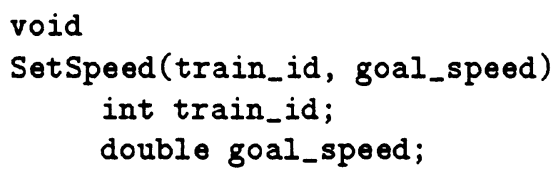

SetSpeed causes the acceleration $a_{\text {train_id }}$ of the train identified by train_id to be assigned the following value.

$$
a_{\text {train_id }}= \begin{cases}+A C C & \text { if goal_speed }>v_{\text {train_id }} \\ 0 & \text { if goal_speed }=v_{\text {train_id }} \\ -A C C & \text { if goal_speed }<v_{\text {train_id }}\end{cases}
$$

The acceleration $a_{\text {train_id }}$ is assigned the value 0 when that train's speed reaches goal_speed, unless $a_{\text {train_id }}$ is changed again or the train derails or collides before the goal speed can be reached.

goal_speed must be non-negative, and train_id must identify an operational train that is not performing an emergency stop; otherwise no action is taken.

\subsubsection{Set Direction}

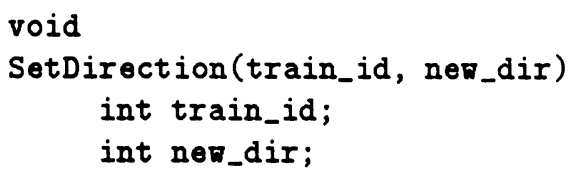

SetDirection causes the direction $d i r_{\text {train_id }}$ train identified by train_id to be assigned the following value.

$$
d i r_{\text {train_id }}= \begin{cases}\text { tail-to-head } & \text { if ner_dir }>0 \\ \text { head-to-tail } & \text { if ner_dir }<0 \\ \text { opposite of dir train_id } & \text { if ner_dir }=0\end{cases}
$$

Here, tail-to-head means that the head end of that train is the front end, and head-to-tail means that the tail end is the front end. 
The direction $d i r_{\text {train_id }}$ of train_id must identify an operational train that satisfies

$$
a_{\text {train_id }}=v_{\text {train_id }}=0
$$

or no action is taken.

\subsubsection{Emergency Stop}

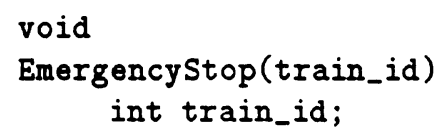

EmergencyStop causes the emergency-stop mode $e s_{\text {train }}$ id of the train identified by train_id to be enabled. This causes an emergency stop to begin immediately. That train's acceleration $a_{\text {train_id }}$ is assigned the value $-A_{\text {emer }}$ until the train's speed $v_{\text {train_id }}$ becomes 0 , at which time $a_{\text {train_id }}$ is assigned the value 0 and $e s_{\text {train_id }}$ is disabled.

train_id must identify an operational train, or no action is taken.

\subsubsection{Station Stop}

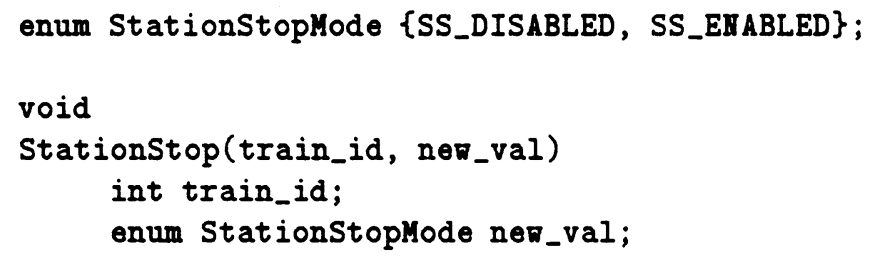

StationStop causes the station-stop mode $s s_{\text {train_id }}$ of the train identified by train_id to be assigned ner_val. A station stop (see Section 2.3.2) begins if an operational train enters a station block with station-stop mode enabled and with speed $v_{\text {train_id }}$ that does not exceed the block's station-stop speed $V S S_{B}$.

train_id must identify an operational train that is not performing an emergency stop; otherwise no action is taken.

\subsection{Queries}

Queries are the only way that a CPI client can obtain information about a layout after the initial-state download has been received. There are four query types: GetBlockOccupancy, GetSritchPosition, GetTrainStatus and GetTrainMotion. These queries return very limited information. Thus, facts 
about the layout's state such as a train's speed and emergency-stop mode must be computed by a CPI client program from a knowledge of past values and of the properties of Trainset railroads. This makes writing process control programs difficult but realistic.

\subsubsection{Block Occupancy}

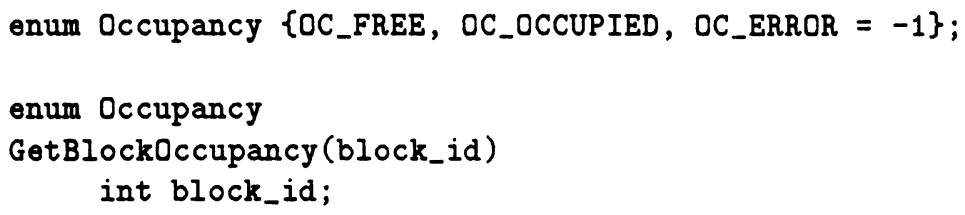

The occupancy status of the indicated block is returned. If block_id is not the ID of a block or if a timeout occurs awaiting a reply from the simulation, OC_ERROR is returned.

\subsubsection{Switch Position}

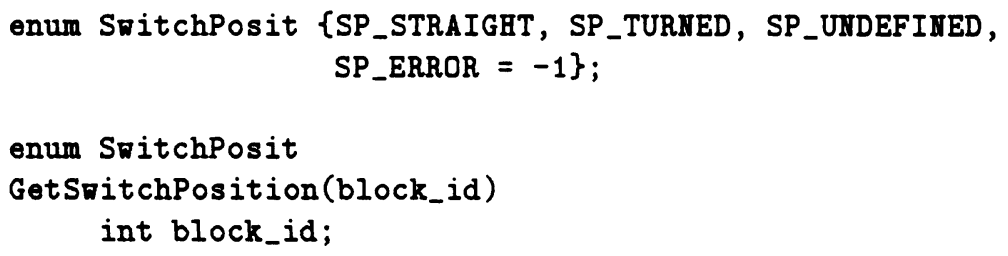

The setting of the indicated switch block is returned. If block_id is not the ID of a switch block or if a timeout occurs awaiting a reply from the simulation, SP_ERROR is returned.

\subsubsection{Train Status}

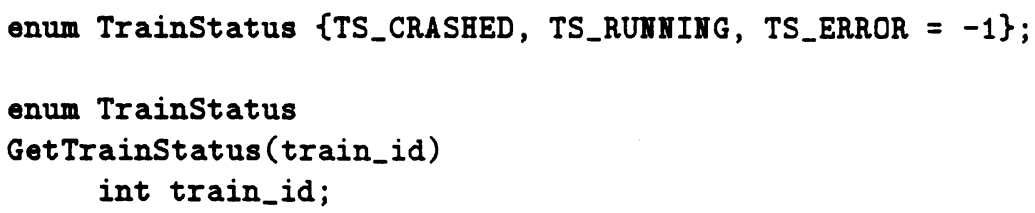

If the indicated train has state operational, TS_RUHIIIIG is returned. If that train's state is derailed or collided, TS_CRASHED is returned. If train_id is not the ID of a train or if a timeout occurs awaiting a reply from the simulation, TS_ERROR is returned. 


\subsubsection{Train Motion}

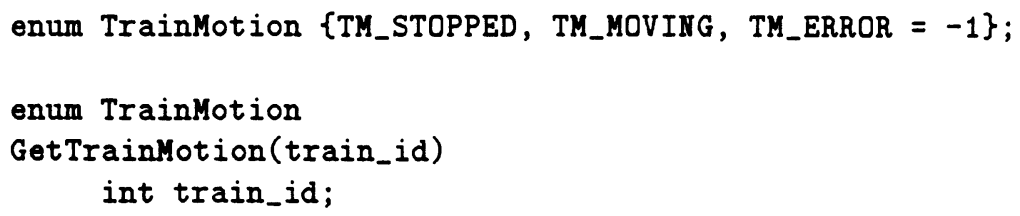

If the indicated train has state operational and at least one of the train's speed $v_{\text {train_id }}$ or acceleration $a_{\text {train_id }}$ is non-zero, TM_MOVING is returned. Otherwise, TM_STOPPED is returned, except that TM_ERROR is returned if train_id is not the ID of a train or if a timeout occurs awaiting a reply from the simulation.

\subsection{Voting}

The voting service supports the writing of fault-tolerant control programs for a Trainset railroad. By default, there is a one-to-one relationship between CPI client commands (satisfying various preconditions, as described in Section 3.3) and layout actions. The voting service makes it possible to require that a simulation receive a specified number $Q_{\text {voting }}$ of commands from different CPI clients within a certain time period $W_{\text {voting }}$ before a layout action can occur.

The behavior of the voting service is governed by the following parameters.

- A voting quorum $Q_{\text {voting (integer). When the voting service is being }}$ employed, a layout action can be generated only if $Q_{\text {voting }}$ commands satisfying the conditions below are received from different CPI clients.

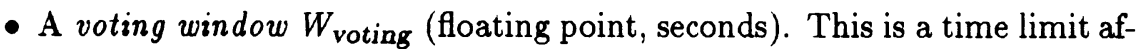
ter which CPI client commands expire. If a command has not contributed to a layout action within $W_{\text {voting }}$ seconds after receipt by a simulation, then that command will have no effect.

- A sequence number seq (integer) that is associated with each CPI command.

We expect $Q_{\text {voting }}>0, W_{\text {voting }} \geq 0$ and $s e q \geq 0$.

The voting service is enabled only for commands with positive sequence numbers. Figuratively speaking, commands with different positive sequence numbers participate in different "elections."

The following paragraphs provide a specification of the voting service.

Sequence number 0 . If a command has sequence number 0 , then that command alone generates a layout action immediately, as described in Section 3.3. The values of $W_{\text {voting }}$ and $Q_{\text {voting }}$ have no effect on such commands. 


\begin{tabular}{ccccc} 
& time & $\begin{array}{c}\text { command } \\
\text { type }\end{array}$ & $\begin{array}{r}\text { client } \\
\text { sequence }\end{array}$ \\
\hline Command A & $7: 22: 06$ & Accel & 2 & 61 \\
B & $7: 22: 13$ & Accel & 1 & 60 \\
C & $7: 22: 17$ & Accel & 3 & 61 \\
D & $7: 22: 19$ & Accel & 1 & 60 \\
E & $7: 22: 25$ & SetSpeed & 2 & 61 \\
F & $7: 22: 30$ & SetSpeed & 3 & 61
\end{tabular}

Figure 3.2: Voting example (positive sequence numbers).

Positive sequence numbers. If a simulation receives $Q_{\text {voting }}$ or more commands with the same positive sequence number seq from different CPI clients during any time period of $W_{\text {voting }}$ seconds or less, then a single layout action is generated. By default, the layout action generated is the action caused (subject to the preconditions listed in Section 3.3) by the last of those commands.

No commands with positive sequence numbers can otherwise contribute to layout actions. Commands with the same sequence number need not have the same arguments nor even the same command type. A command can contribute to the generation of at most one layout action.

For example, suppose that $W_{\text {voting }}=10 \mathrm{sec}, Q_{\text {voting }}=2$ and that the commands in Figure 3.2 are received by a simulation. Then only one layout action will be performed by that simulation as of the time 7:22:30. That layout action will be $\mathrm{E}$, occurring at time 7:22:25. The quorum of commands consists of $\mathrm{C}$ and $\mathrm{E}$.

The values of $Q_{\text {voting }}$ and $W_{\text {voting }}$ are determined when a Trainset railroad simulation is invoked, and cannot be changed during a simulation. The default value for $Q_{\text {voting }}$ is 2 , and the default value for $W_{\text {voting }}$ is 5 seconds.

Sequence numbers can be modified dynamically by a control program. The ACI provides an internal sequence-number variable $s e q_{A C I}$ whose value is automatically sent with each command. The value of the variable $s e q_{A C I}$ remains constant unless explicitly modified, and the initial value is 0 .

In order to employ the voting service, it is necessary to start a simulation with $Q_{\text {voting }}>1$ and to send commands that have positive sequence numbers. The ACI provides two functions for modifying the value of a sequence number. 


\subsubsection{New Sequence Number}

int

NewSeqHumber ()

The sequence number is incremented and the new value is returned.

\subsubsection{Set Sequence Number}

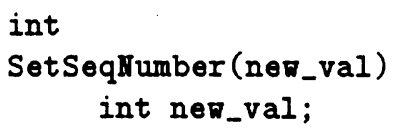

If new_val is non-negative, the sequence number seq is assigned nev_val, and that value is returned. Otherwise, seq is left unchanged and -1 is returned.

\subsection{Timer Facilities}

The ACI layer provides procedures that implement a simple timer service in which time quantities are represented as seconds in floating point. These routines affect a program that calls them; they do not themselves interact with a Trainset railroad. Alternative time-management routines or direct system calls may be used instead of these timer procedures when writing programs that use the ACI. Thus, use of the ACI timer facilities is optional.

The five timer procedures are InitTimer, GetTimer, AwaitTimer, CancelTimer and sleep.

\subsubsection{Initialize Timer}

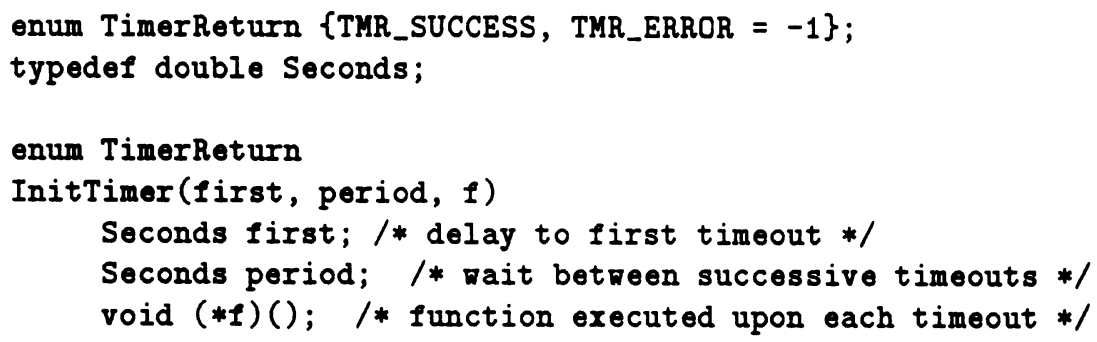

InitTimer initializes the timer mechanism to execute the function $f()$ after first seconds and every period seconds thereafter. If 1 irst is zero, the timer is immediately disabled. If period is zero, the timer executes $f()$ at most once. 
If the value zero is specified instead of a function $f$, then nothing is done on timeouts except to reset the timer as appropriate. TMR_SUCCESS is returned if the operation succeeds. If a time argument is negative or a low-level error occurs, TMR_ERROR is returned.

A call to InitTimer overrides any prior timer setting. Thus, there is no provision for more than one timer being in effect at any time. The timers used in other ACI functions, including GetDornload, the ACI queries and Sleep, do not interfere with InitTimer.

\subsubsection{Get Timer Values}

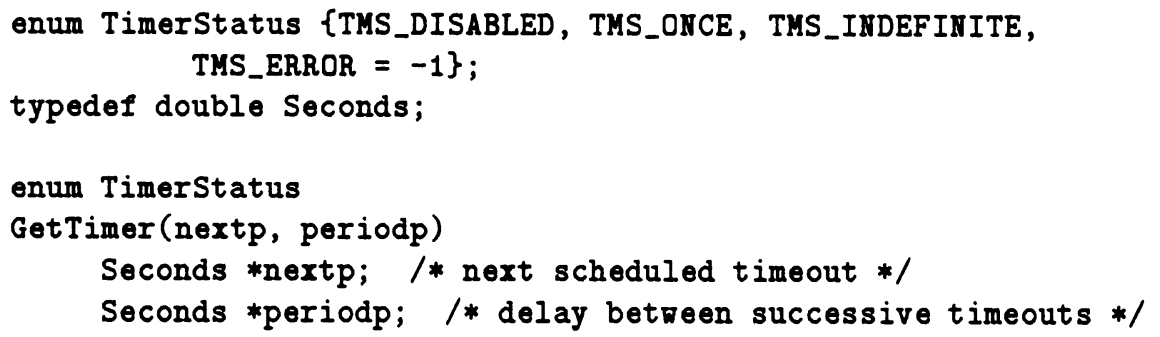

GetTimer stores the seconds remaining until the next timer expiration in *nextp and stores the current period between timer expirations in *periodp. The return value is TMS_DISABLED if the timer is currently disabled, TMS_OrCE if the timer is scheduled to expire once only, TMS_IMDEFIIITE if the timer is scheduled to continue indefinitely and TMS_ERROR if a low-level error occurs.

\subsubsection{Await for Timer}

$$
\text { void }
$$

AwaitTimer()

AvaitTimer suspends the calling process until an ACI timer expiration or another operating system signal occurs.

\subsubsection{Cancel Timer}

enum TimerReturn \{TMR_SUCCESS, TMR_ERROR $=-1$;

enum TimerReturn

Canceltimer() 
CancelTimer cancels an ACI timer. TMR_SUCCESS is returned if the operation succeeds. Canceltimer fails if a low-level error occurs, in which case TMR_ERROR is returned.

\subsubsection{Sleep}

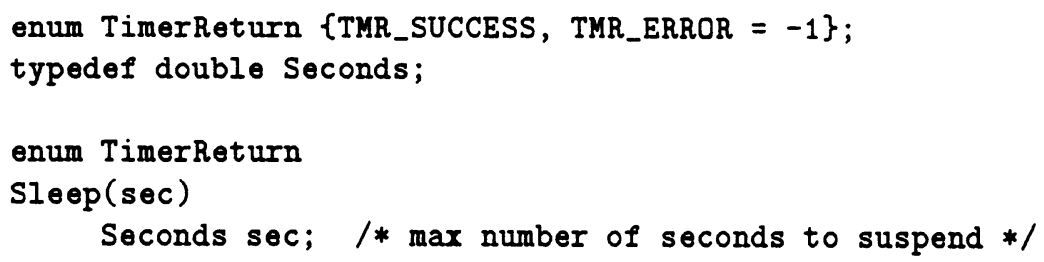

Sleep suspends the calling process for indicated number of seconds. The value TMR_SUCCESS is returned if the suspension was not interrupted before the time limit expired. Otherwise, TMR_ERROR is returned.

Sleep does not interfere with the operation of InitTimer. Thus, these functions may be used together, e.g., to set up a polling loop with a timeout. 


\section{Chapter 4}

\section{Low-Level Interface (LLI)}

\subsection{Introduction}

The Low-Level Interface (LLI) consists of predefined message formats and related facilities for communicating with a Trainset railroad simulation. This layer is used to implement the ACI layer in the Control Program Interface (see Figure 3.1).

This chapter gives a sketch of the LLI layer. A reading knowledge of the $\mathrm{C}$ programming language is assumed. The rest of this chapter includes the following sections.

- Section 4.2 is external documentation for the source file cpi.h (see Appendix C.1). The internal structure of that file is described, and the source lines that pertain to the acceleration command are discussed as examples.

- Section 4.3 describes the steps necessary for a client to connect and identify itself to a Trainset railroad simulation.

- Sections 4.4 and 4.5 briefly list the message formats that are used for initial-state downloads, commands and queries. A knowledge of the ACI layer (Chapter 3) is assumed.

For a programming example that uses the LLI programming layer, see the implementation of the ACI layer in the source file aci.c (Appendix C.2).

\subsection{Messages}

In the LLI layer, a control program interacts with a Trainset railroad simulation by sending and receiving messages. An LLI message is an ASCII character string that is organized according to one of the message formats defined in 
1

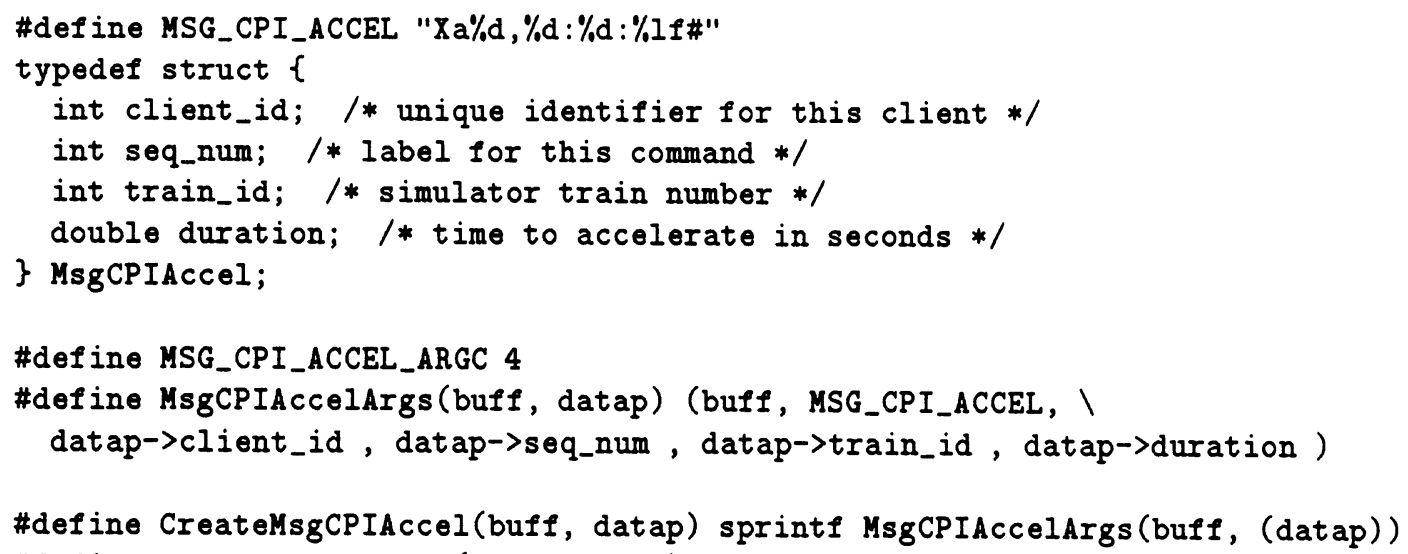

Figure 4.1: Excerpt from cpi.h relating to acceleration command.

cpi.h. Each such message format consists of fields for representing data, together with an identifying header and delimiter characters.

For example, consider Figure 4.1, an excerpt from cpi.h. Line 1 defines a message format called MSG_CPI_ACCEL. That message format has the following syntax.

$$
\mathrm{Xa}\langle\text { integer }\rangle,\langle\text { integer }\rangle:\langle\text { integer }\rangle:\langle\text { float }\rangle \#
$$

There are four fields. The notations (integer $\rangle$ and $\langle$ float $\rangle$ indicate ASCII representations of integer and floating point numbers. The total length of a message depends on the lengths of the data in its fields.

In general, for each message format in cpi.h there is a type definition of an associated data structure whose components correspond to the fields of that message format, in the same order. ${ }^{1}$ For MSG_CPI_ACCEL, the associated data structure is called MsgCPIAccel. See the lines near 2 in Figure 4.1.

A comparison of the message format MSG_CPI_ACCEL and the documented data structure MsgCPIAccel shows that the first (integer) field in that message format holds the client ID ${ }^{2}$ the second $\langle$ integer $\rangle$ field holds the voting sequence number of an Accelerate command, etc.

For each message format, a macro has been defined that produces a string in that message format, using values stored in a variable of the associated data

\footnotetext{
${ }^{1}$ Four CPI message formats are associated with basic data types that are not explicitly defined as structures in cpi.h. These are MSG_CPI_IIIT (string), MSG_ACK (string), MSG_CPI_DOUILD DOIE (integer) and MSG_QUIT_ALL (string).

${ }^{2} \mathrm{~A}$ unique client $\mathrm{ID}$ is assigned to each CPI client when that client connects to a simulation, as described below.
} 
type for the fields. Another macro has been defined for parsing such a message into a variable the associated data type. These macros are provided as a convenience. In the case of MSG_CPI_ACCEL, the creation macro is called CreateMsgCPIAccel and is defined by line 3 in Figure 4.1. The messageparsing macro is ParselsgCPIAccel defined on line 4 .

In general, for any such macro associated with a message format:

- There are two arguments, a character string buffer holding the message and a pointer to a variable of the associated data structure type holding the values.

- The run-time replacement value of such a macro is the length of the resulting message, not counting a null byte that is placed at the end of that message string. ${ }^{3}$

Thus, the macro CreateMsgCPIAccel behaves like a function with the following heading.

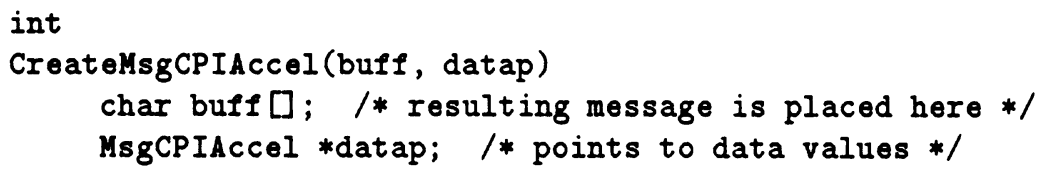

\subsection{Initiating Communication}

Establishing communication between a client and a Trainset railroad simulation requires two steps.

1. Connection. Create a network connection between that client and that simulation. A simulation provides well-known ports for supported communication protocol families (e.g., TCP/IP, DECnet) and is capable of socket or stream transmissions in each case. The function GetSimport defined in the source file lib/syslocal.c returns the well-known port, given a base port (provided in lib/syslocal.h), a host name and an integer simnum that distinguishes between multiple simulations running on the same host. Use standard techniques to open one bidirectional communication line.

2. Registration. Send a registration message to that simulation to declare a client type (CPI or monitor) and receive a client ID. A registration message for a CPI client has the message format MSG_CPI_IIIIT

$$
\text { CPI 〈one space〉〈string } \%
$$

\footnotetext{
${ }^{3}$ Sending a terminating null byte to a simulation as part of a message is optional.
} 
where 〈string is any sequence of characters that are not the percent sign $\%$ and is conventionally the file name of that client's executable. The simulation replies with an acknowledgement message in the format MSG_ACK, as follows.

$$
\text { ack 〈one space〉〈integer〉\# \% }
$$

The 〈integer $\rangle$ field in that message represents the unique client ID number to be used in all commands and queries from that client to the simulation.

The macros CreateMsgCPIInit and ParseMsgAck may be used if desired.

We assume that each client creates only one connection with a simulation.

For the remainder of this chapter, message formats will be cited by name only, omitting mention of syntax, related macros, etc. See Appendix C.1 for syntax details.

\subsection{Initial-State Download}

After initiating communication with a simulation, a CPI client can receive a report of the current global state of the railroad being simulated. Such a report is called a download.

To request a download, a control program must send a message to a simulation using the message format MSG_CPI_DOWHLD_REQUEST. The acknowledgement from the simulation is the download itself. The download consists of messages in the following DOWHLD formats.

- MSG_CPI_DOWHLD_GEIERAL transmits constants that describe a railroad in general, e.g., the number of blocks.

- MSG_CPI_DOWHLD_TRAII describes attributes of trains. One message in this format is sent per train.

- MSG_CPI_DOWHLD_BLOCK describes a block. MSG_CPI_DOWHLD_LIHK gives block attachments for a block as described in Section 3.2. One message in each of these formats is sent per block.

- MSG_CPI_DOWHLD_DONE marks the end of a download.

Only one download request is honored by a simulation per client connection.

\subsection{Commands and Queries}

- MSG_CPI_SET_SWITCH, MSG_CPI_ACCEL, MSG_CPI_DECEL, MSG_CPI_SET_SPEED, MSG_CPI_SET_DIR, MSG_CPI_EMER_STOP and

MSG_CPI_STA_STOP request the commands described in Section 3.3. No acknowledgement message is sent in response to such a command. 
- MSG_CPI_BLOCK_OCC requests a block's occupancy status. A simulation responds with a message " 4 " if that block is occupied or "f" (for "free") if it is not occupied.

- MSG_CPI_SWITCH_POSIT asks for the current setting of a switch block. The response is " $s$ " for straight, " $t$ " for turned, or " $\mathrm{u}$ " for undefined.

- MSG_CPI_TRAIH_STATUS inquires about a train's status. A simulation responds with " $r$ " for running (operational state) or " $c$ " for crashed (collided or derailed states).

- MSG_CPI_TRAII_MOTION queries whether a train is moving. The response is " $s$ " for stopped or " $\mathrm{m}$ " for moving. A train is moving if it is operational and at least one of its speed and acceleration is non-zero. A train is stopped if it is not moving.

\subsection{Quit Message From the Simulator}

Just before a Trainset simulation exits normally, it sends a message in the format MSG_QUIT_ALL to all of its clients. Receipt of this message is a certain indicator that a simulation has finished.

\footnotetext{
${ }^{4}$ Note: One-character return strings such as " 0 " are sent as two-byte messages from the simulator, including the terminating null byte.
} 


\section{Appendix A}

\section{Constants Used by Trainset}

\begin{tabular}{clcl}
$\begin{array}{c}\text { Constant } \\
\text { name }\end{array}$ & \multicolumn{1}{c}{ Description } & $\begin{array}{c}\text { Typical } \\
\text { value }\end{array}$ & $\begin{array}{c}\text { Page } \\
\text { references }\end{array}$ \\
\hline$A C C$ & Standard acceleration rate for trains & $1.5 \mathrm{~m} / \mathrm{sec}^{2}$ & $\mathbf{3 6}, 40,41,42$ \\
$A_{\text {emer }}$ & Emergency-stop acceleration rate for trains & $2.5 \mathrm{~m} / \mathrm{sec}^{2}$ & $36, \mathbf{3 7}, 40,43$ \\
$L E N_{\text {cross }}$ & Length of a cross block & $35 \mathrm{~m}$ & $\mathbf{3 3}$ \\
$L E N_{\text {join }}$ & Length of a join block & $35 \mathrm{~m}$ & $\mathbf{3 2}$ \\
$L E N_{\text {switch }}$ & Length of a switch block & $35 \mathrm{~m}$ & $\mathbf{3 4}$ \\
$L_{\boldsymbol{B}}$ & Length of block B & 35 to $300 \mathrm{~m}$ & $\mathbf{3 0}$ \\
$L_{T}$ & Length of train $T$ & 150 to $500 \mathrm{~m}$ & $\mathbf{3 5}$ \\
$M N_{B}$ & Minimum speed limit on block $B$ & $0 \mathrm{~m} / \mathrm{sec}$ & $\mathbf{3 0}, 36,40$ \\
$M X_{\boldsymbol{B}}$ & Maximum speed limit on block $B$ & $60 \mathrm{~m} / \mathrm{sec}$ & $\mathbf{3 0}, 36,40$ \\
$N_{\text {blocks }}$ & Number of blocks in a layout & 5 to 50 & $\mathbf{4 0}$ \\
$N_{\text {trains }}$ & Number of trains in a layout & 1 to 3 & $\mathbf{4 0}$ \\
$Q_{\text {voting }}$ & Voting quorum & 2 & $40, \mathbf{4 5}, 46$ \\
$T_{\text {switch }}$ & Time for a switch block to change settings & $2 \mathrm{sec}$ & $\mathbf{3 3}, 40,41$ \\
$V S S_{B}$ & Station stop speed for a station block & $30 \mathrm{~m} / \mathrm{sec}$ & $\mathbf{3 4}, 37,40,43$ \\
$W_{\text {voting }}$ & Voting window & $5 \mathrm{sec}$ & $40, \mathbf{4 5}, 46$
\end{tabular}

\footnotetext{
${ }^{1} \mathrm{~A}$ boldface number indicates the primary reference for a symbol.
} 


\section{Appendix B}

\section{Code Listings: ACI}

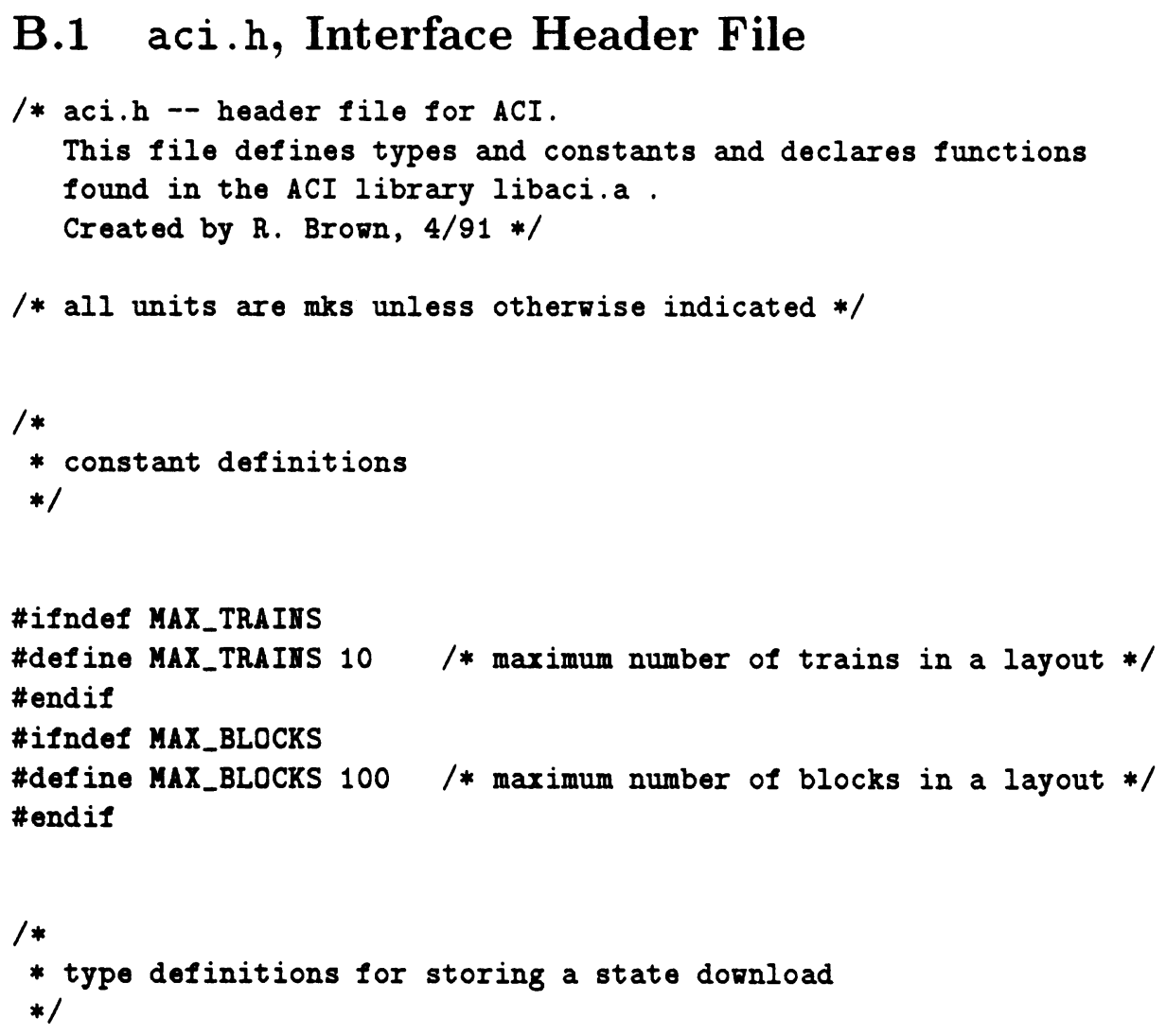




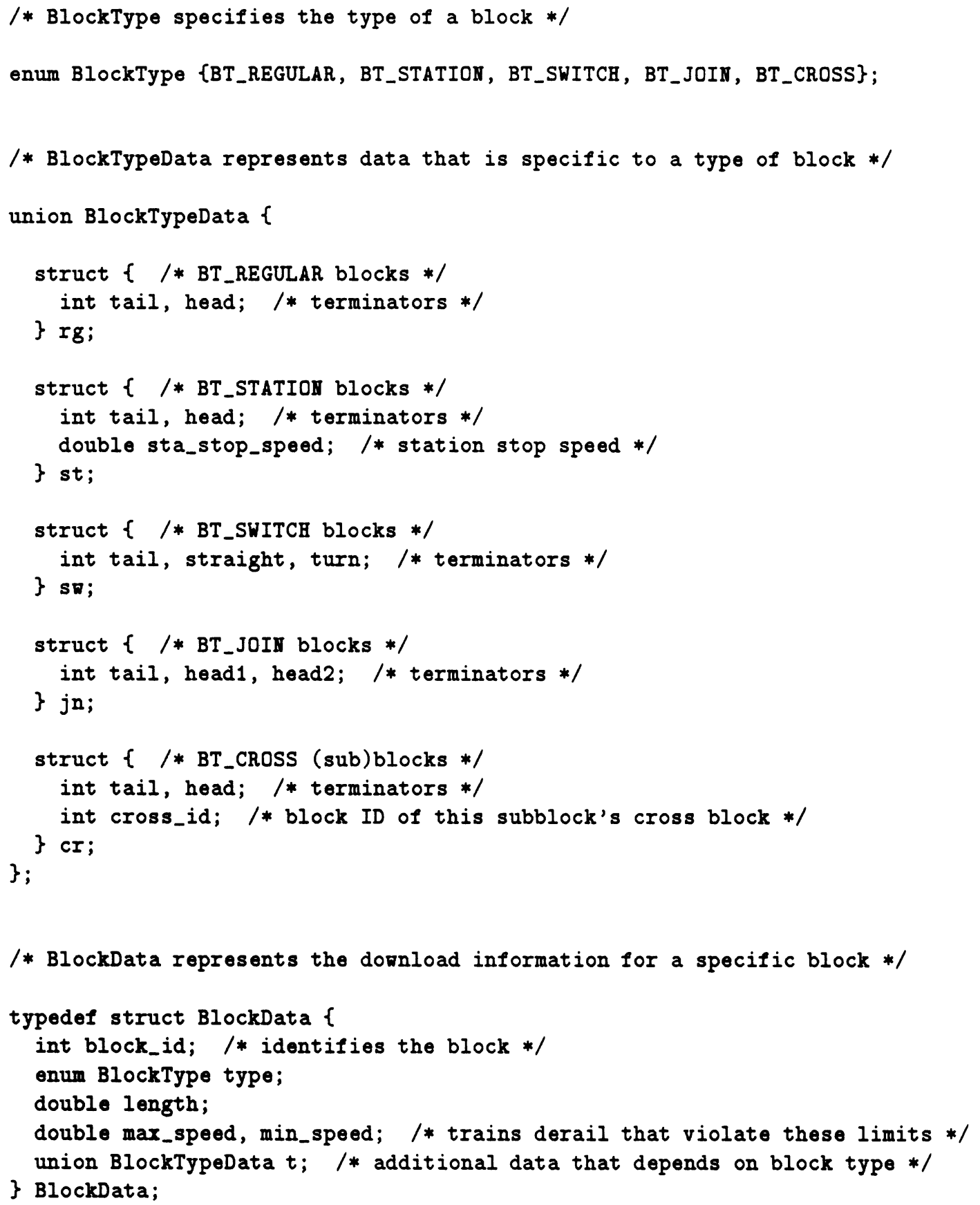




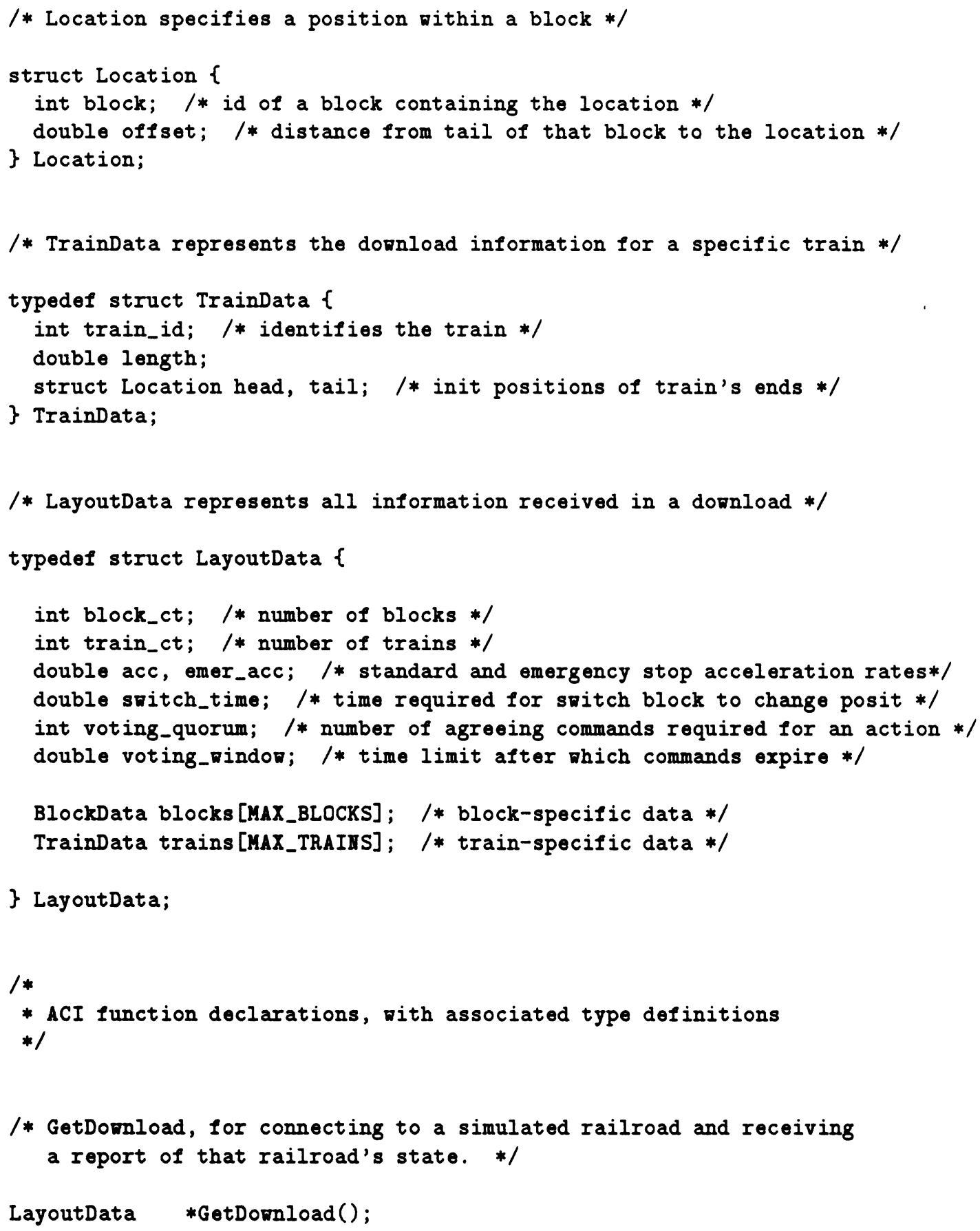




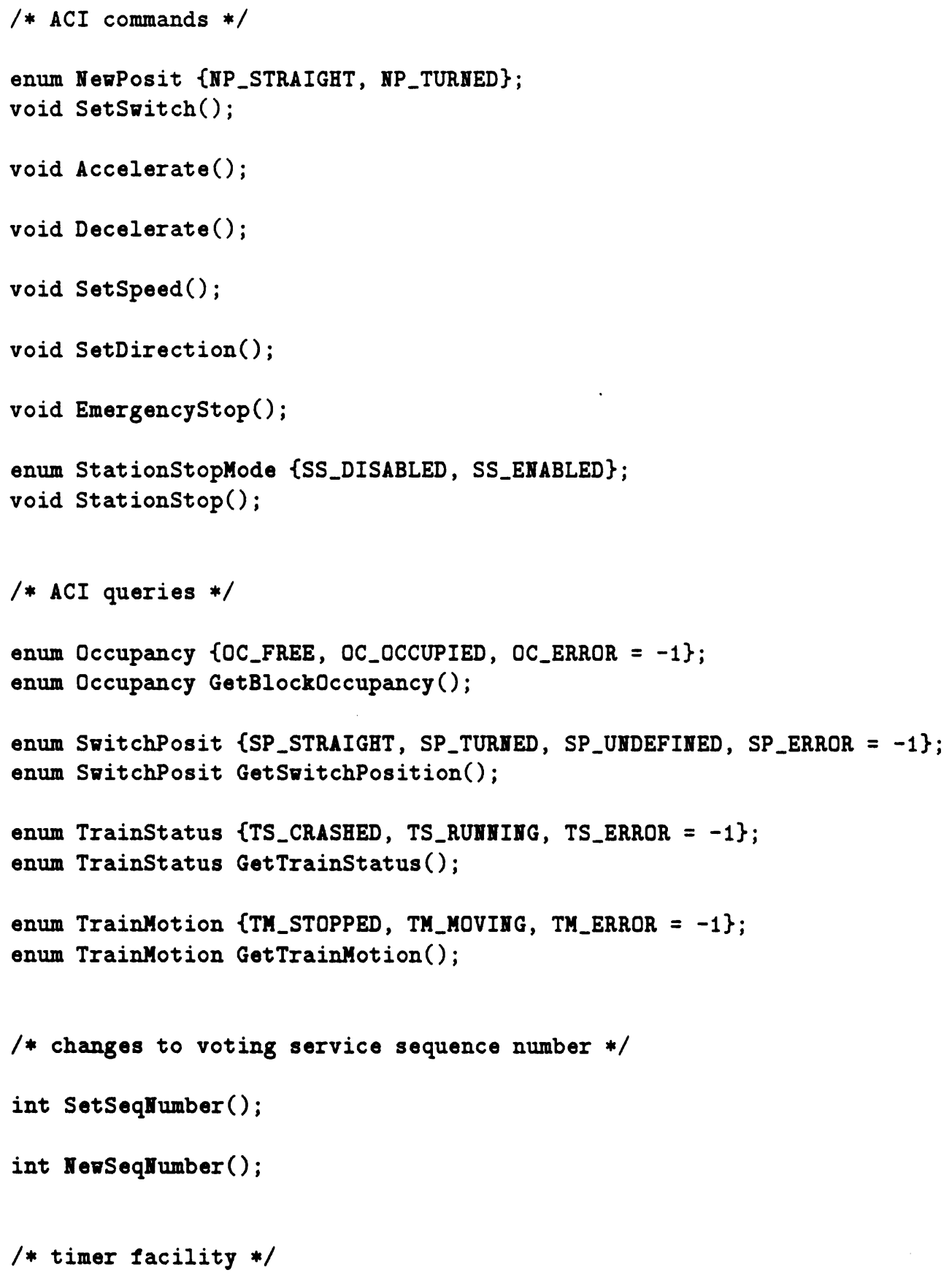




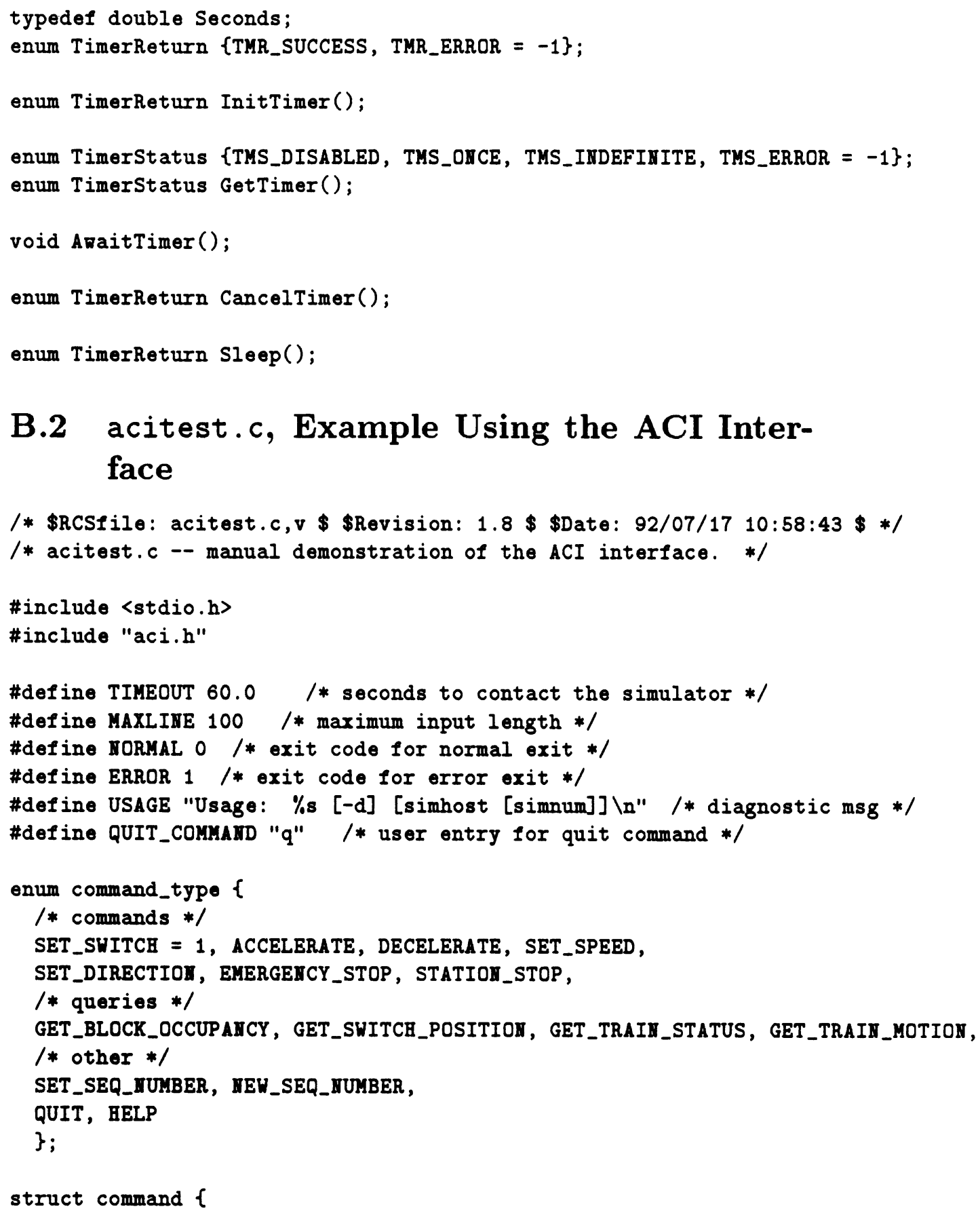




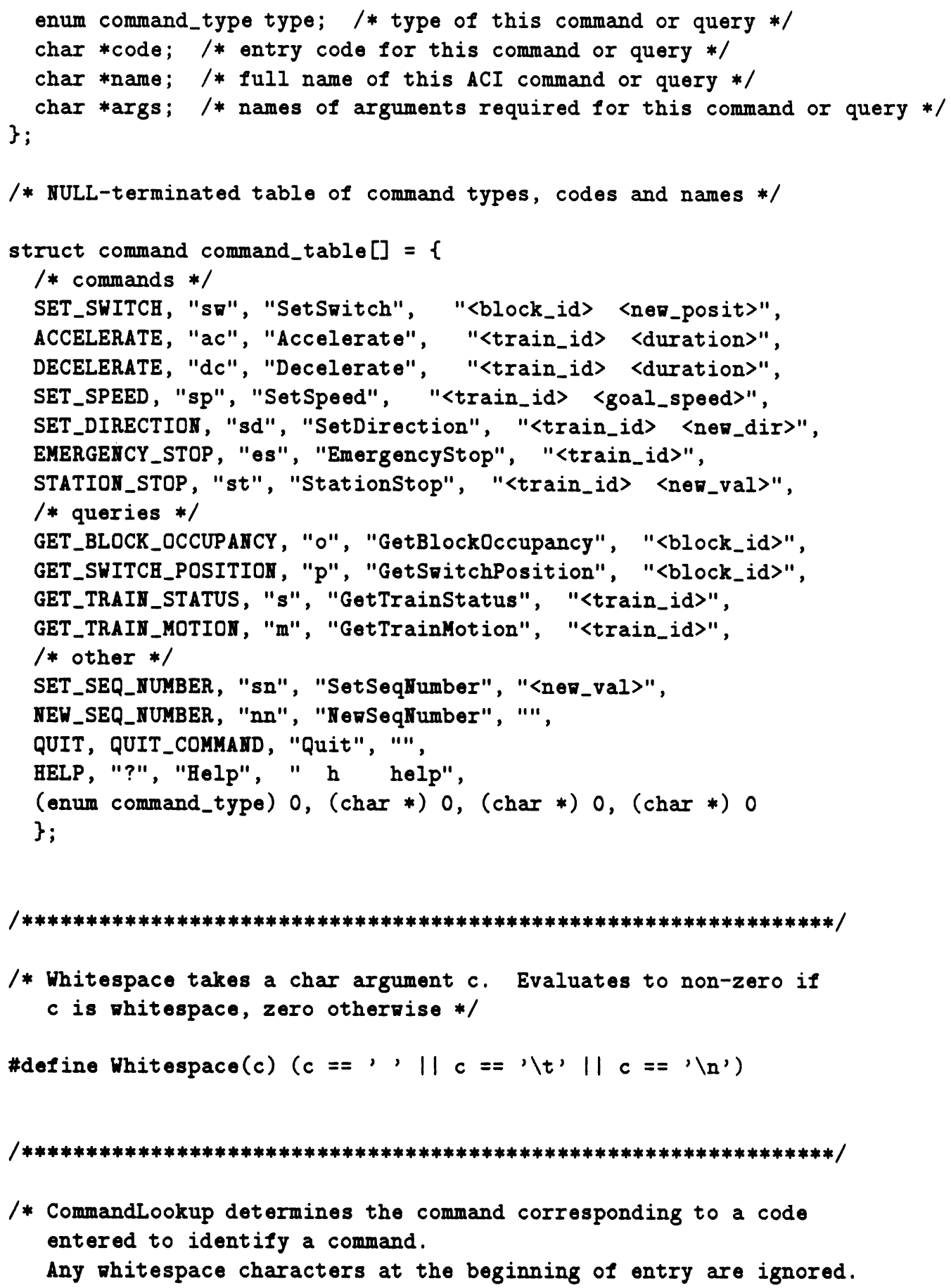

$/ * * * * * * * * * * * * * * * * * * * * * * * * * * * * * * * * * * * * * * * * * * * * * * * * * * * * * * * * * * * * * * * * * /$

/* Whitespace takes a char argument c. Evaluates to non-zero if c is vhitespace, zero othervise */

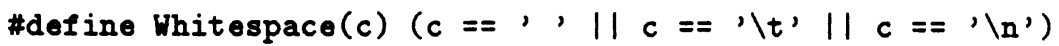


Returns the index in the table of the indicated command, or

-1 if the code was empty or the given entry code was not found. */

int

CommandLookup (entry)

char *entry; /* entry code to be interpreted as a command */

\{

struct command *p; /* pointer into command table */

while (*entry \&\& Whitespace(*entry))

entry++;

if (!*entry)

return $(-1)$;

/* entry is non-empty string beginning with a non-whitespace char */

if (*entry $==$ ' $h$ ')

entry = "?";

/* if request is for Help then entry has value "?" */

for ( $p=$ command_table; $p$->type; $p++)\{$

if (!strcmp (entry, $p->\operatorname{cod} \theta))\{$

\} return ( $p$ - command_table);

/* entry does not match a code in the table */

printf (" no match found $\backslash n^{\prime \prime}$ );

\}

return $(-1)$;

$/ * * * * * * * * * * * * * * * * * * * * * * * * * * * * * * * * * * * * * * * * * * * * * * * * * * * * * * * * * * * * * * * * /$

/* PrintDownload displays every field received in a download */

void

PrintDownload (dp, simhost, simnum)

LayoutData *dp; /* data received from download */

char *simhost; /* name of the host system running the simulator */

\{

int simnum; /* identifier for a running simulation on that host */

int $i ; / *$ loop control */

TrainData *tp; /* utility pointer to data for a specific train */

BlockData *bp; /* utility pointer to data for a specific block */ 


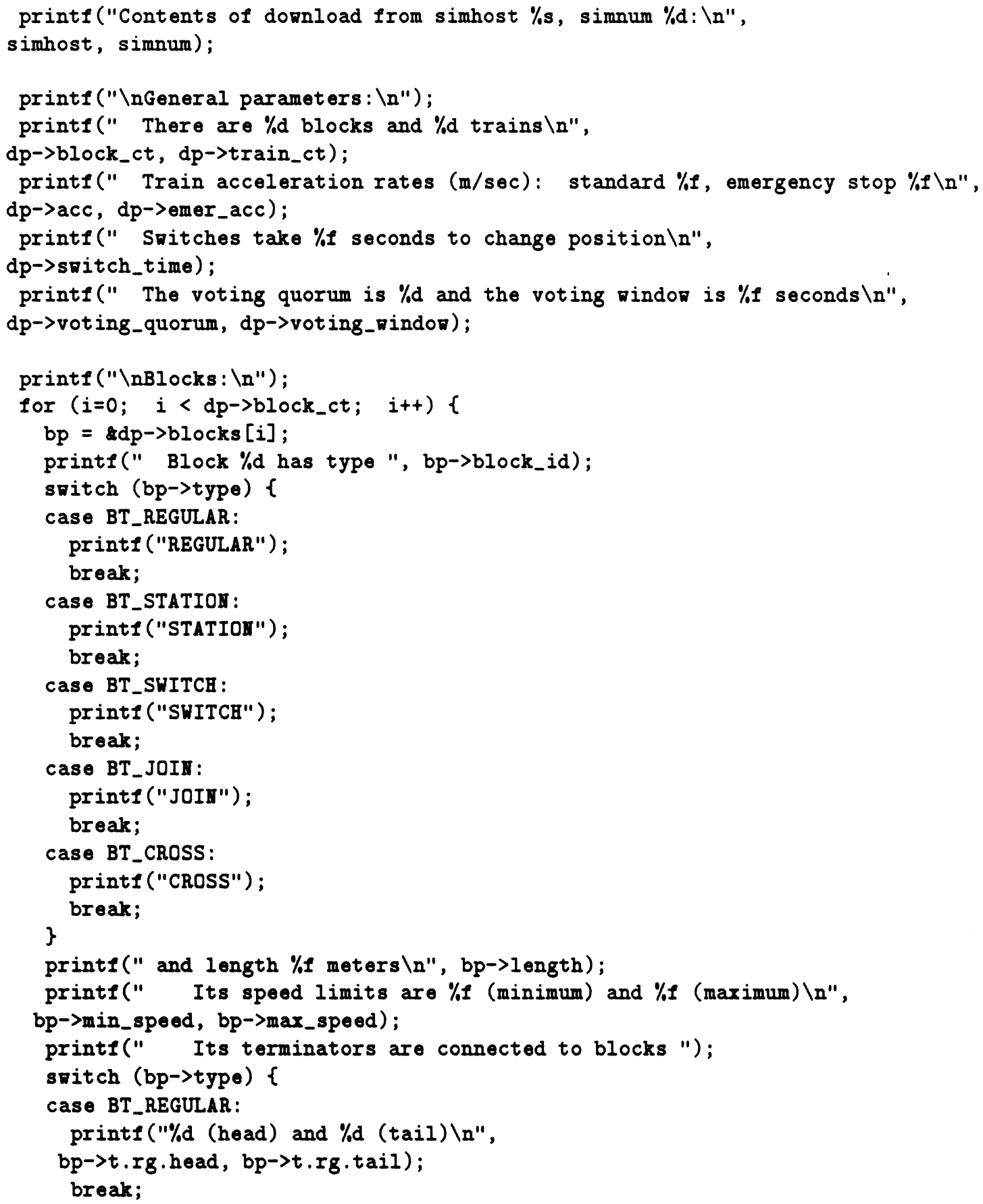




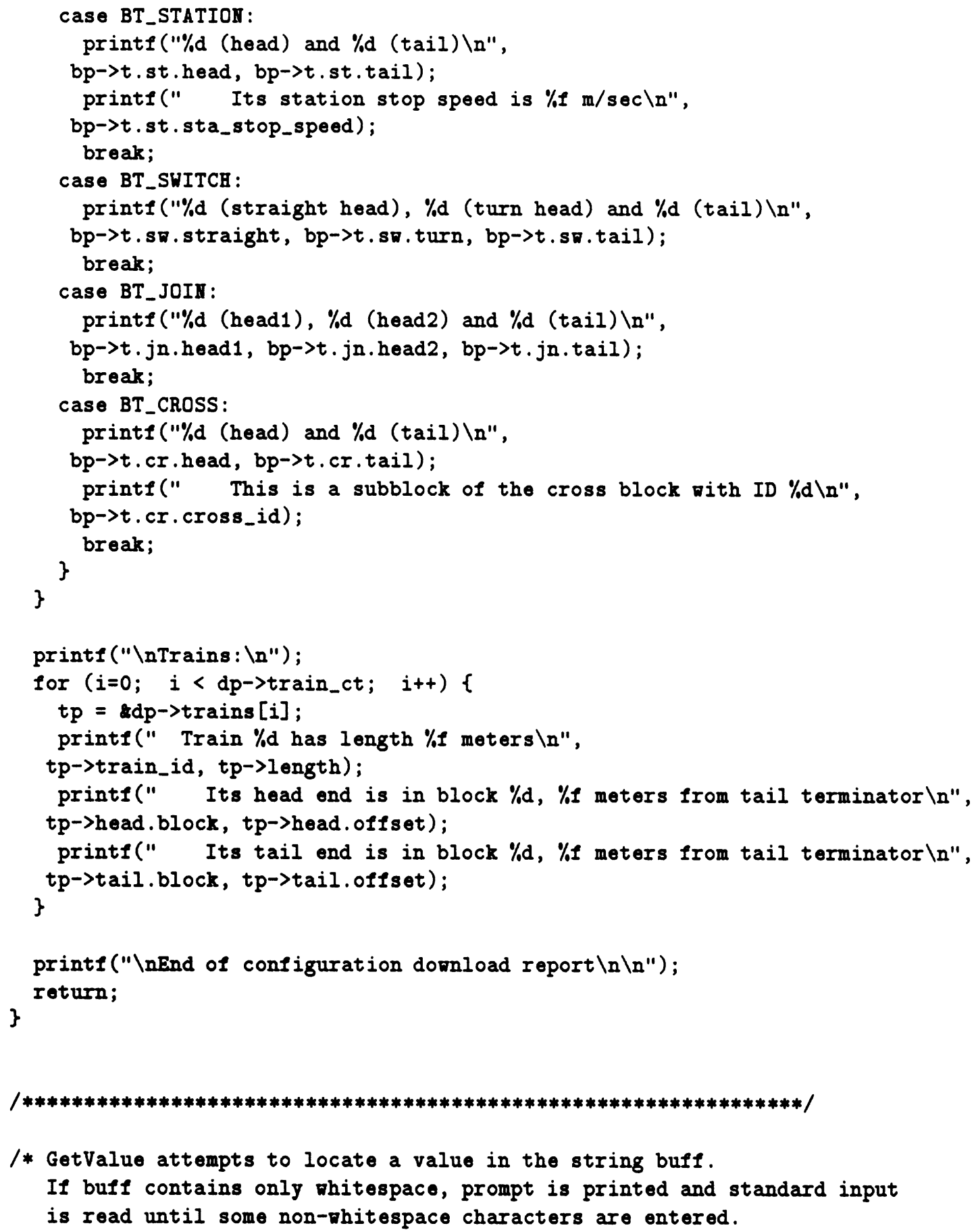

$/ * * * * * * * * * * * * * * * * * * * * * * * * * * * * * * * * * * * * * * * * * * * * * * * * * * * * * * * * * * * * * * * * /$

/* GetValue attempts to locate a value in the string buff.

If buff contains only whitespace, prompt is printed and standard input is read until some non-whitespace characters are entered. 
If a value matching the printf format fmt is found in buff or stdin, then its value is placed in *valp and a pointer is returned that points to the first whitespace character after the value.

If the next non-whitespace characters do not scan according to fmt, an error message requesting user to try again is printed and

IIULL is returned. */

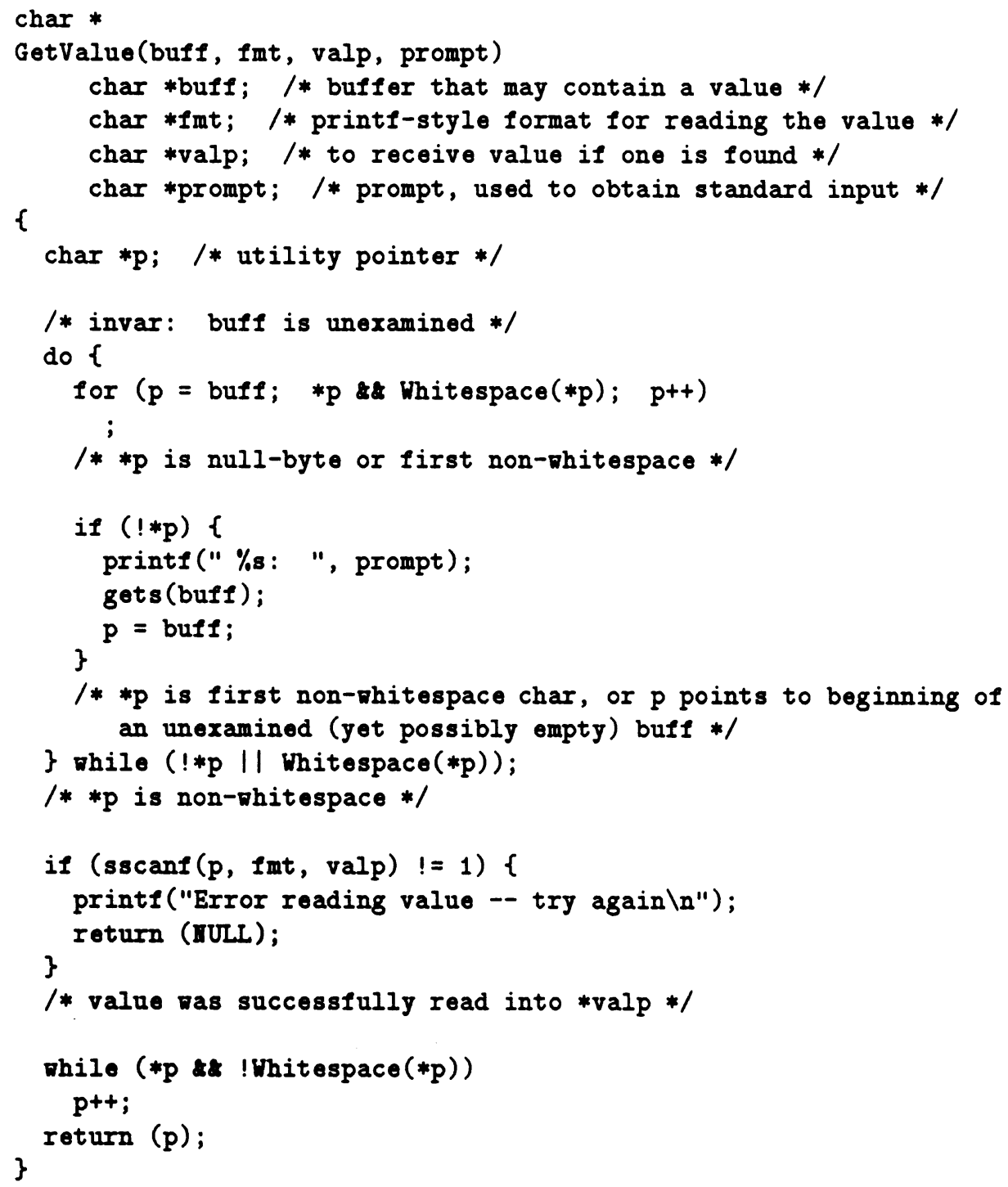




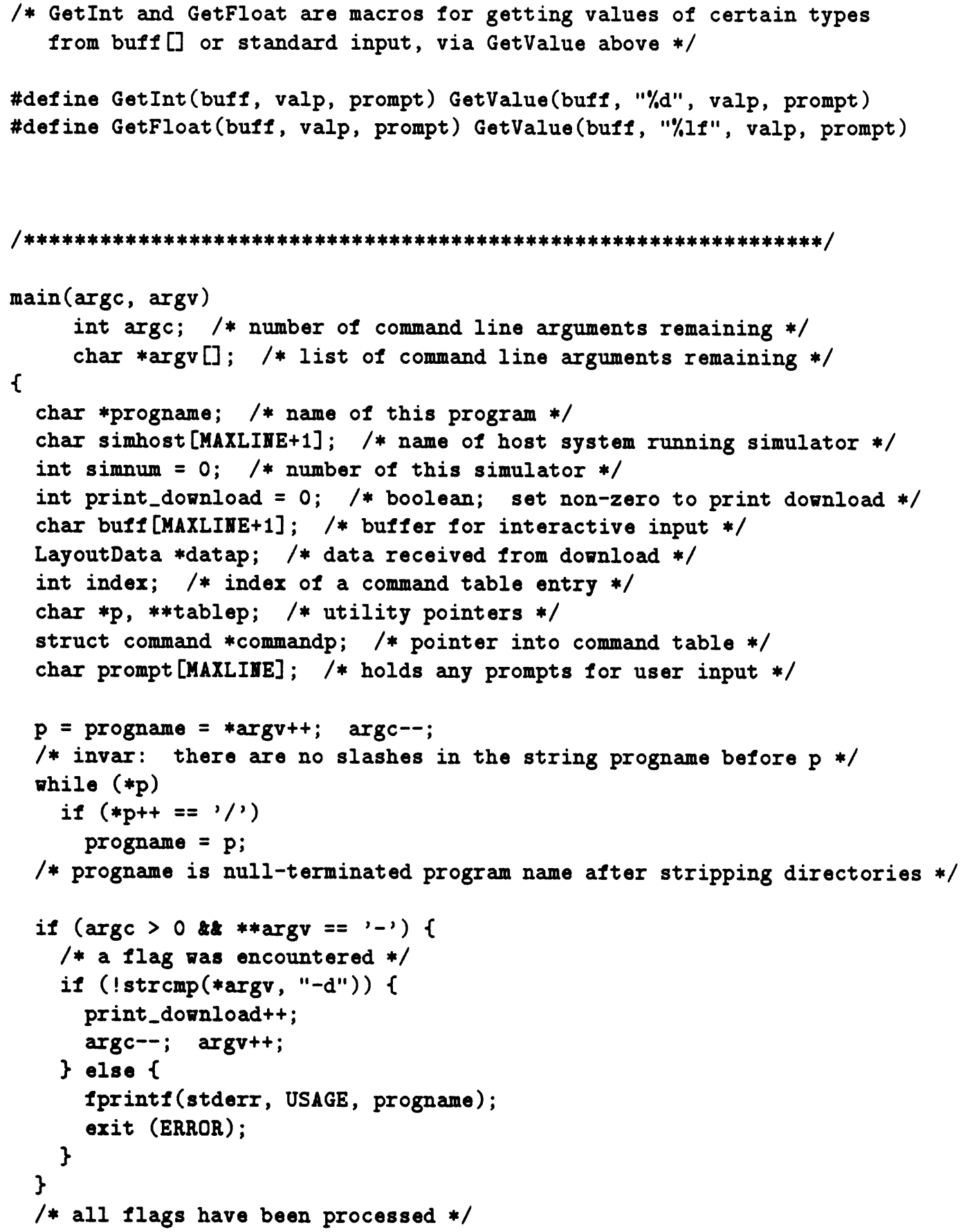




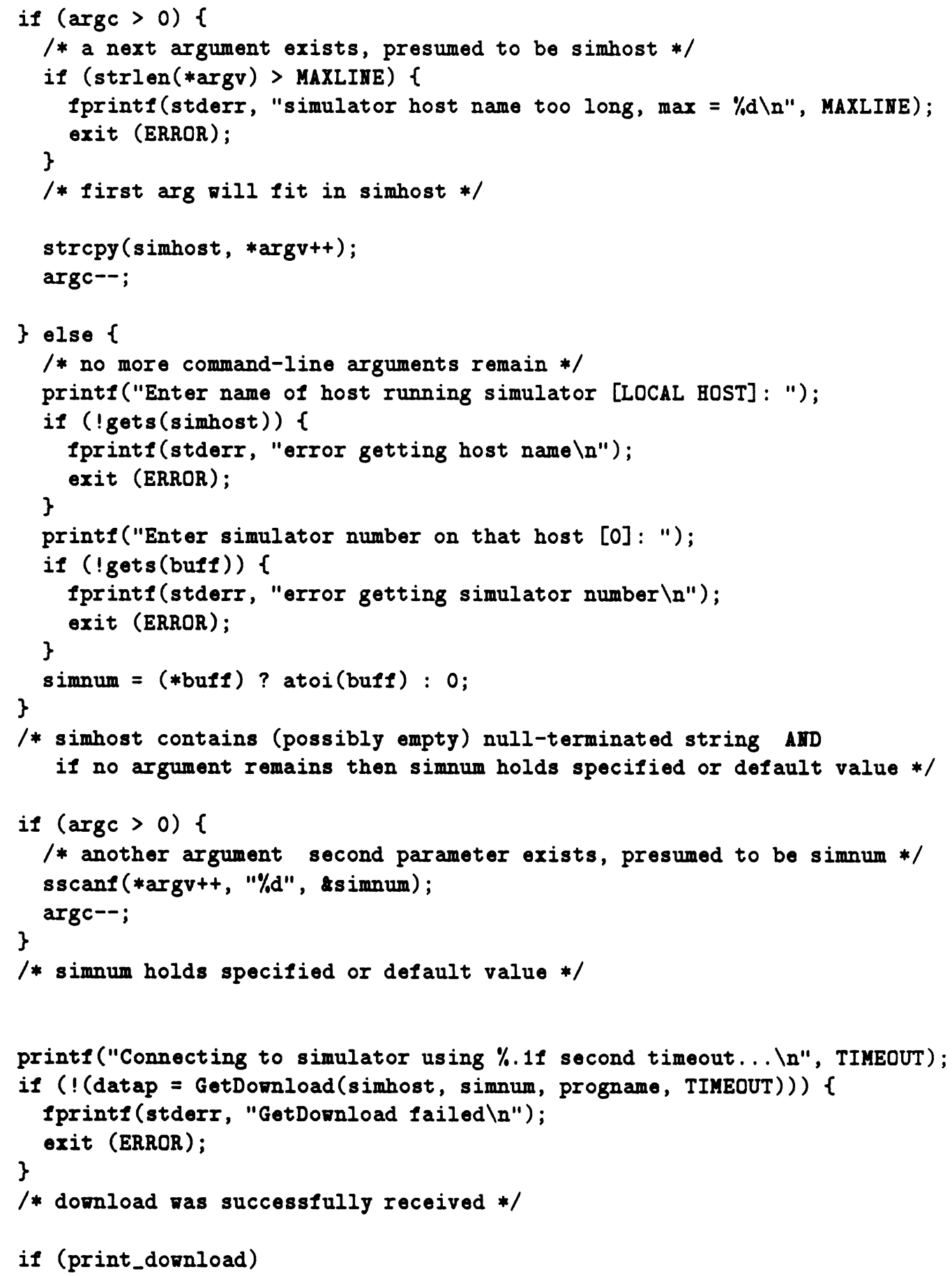




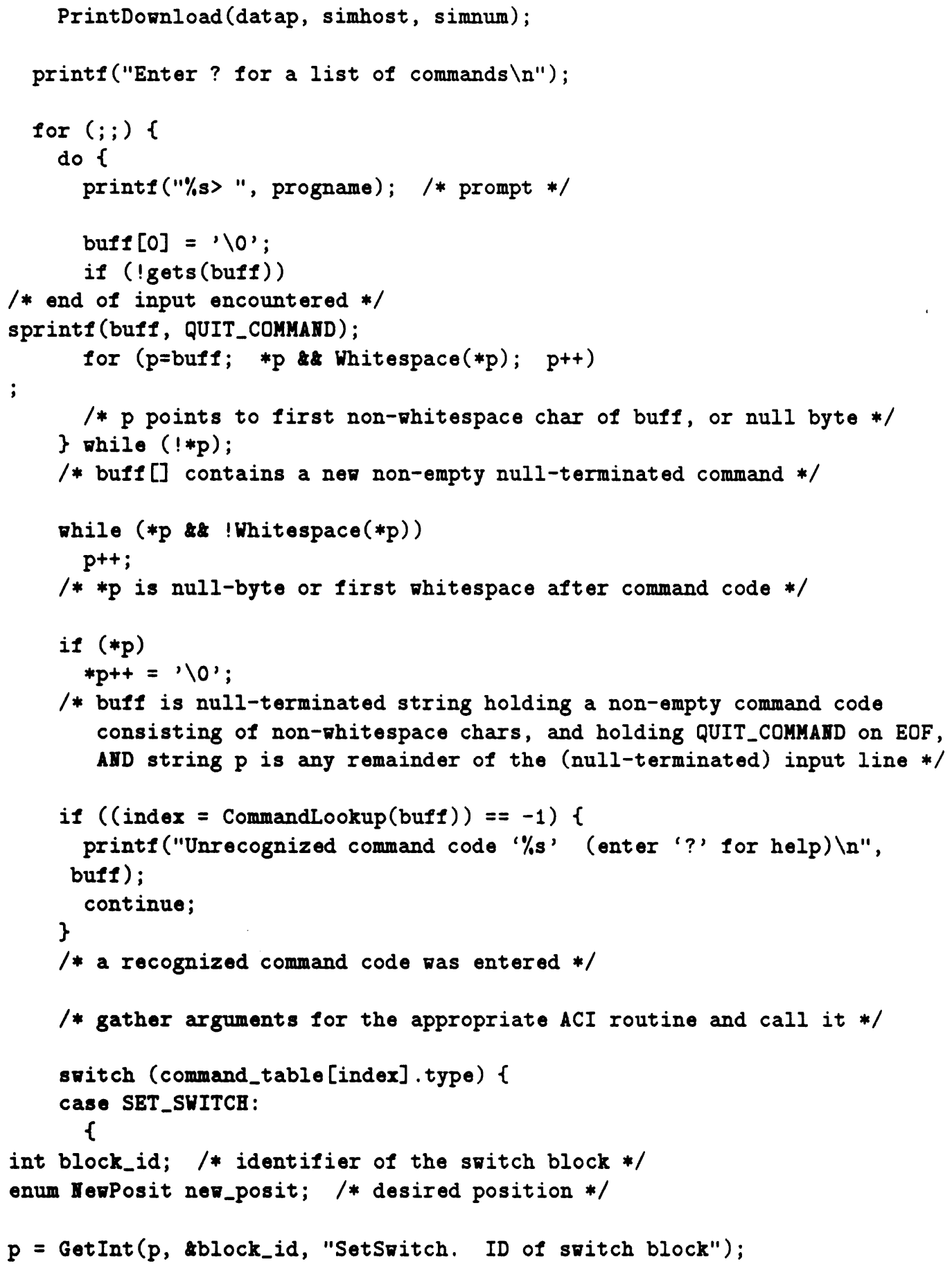




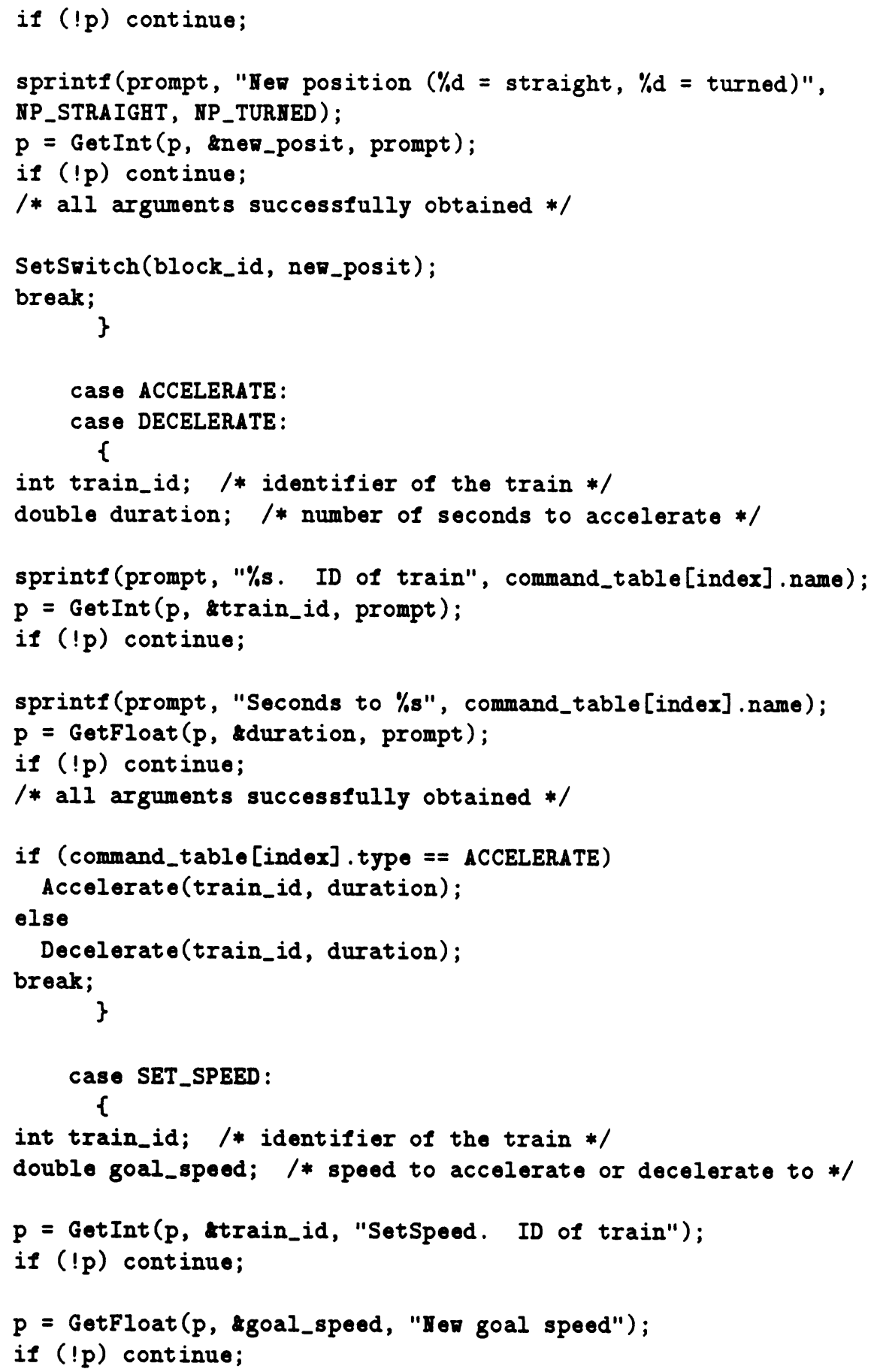




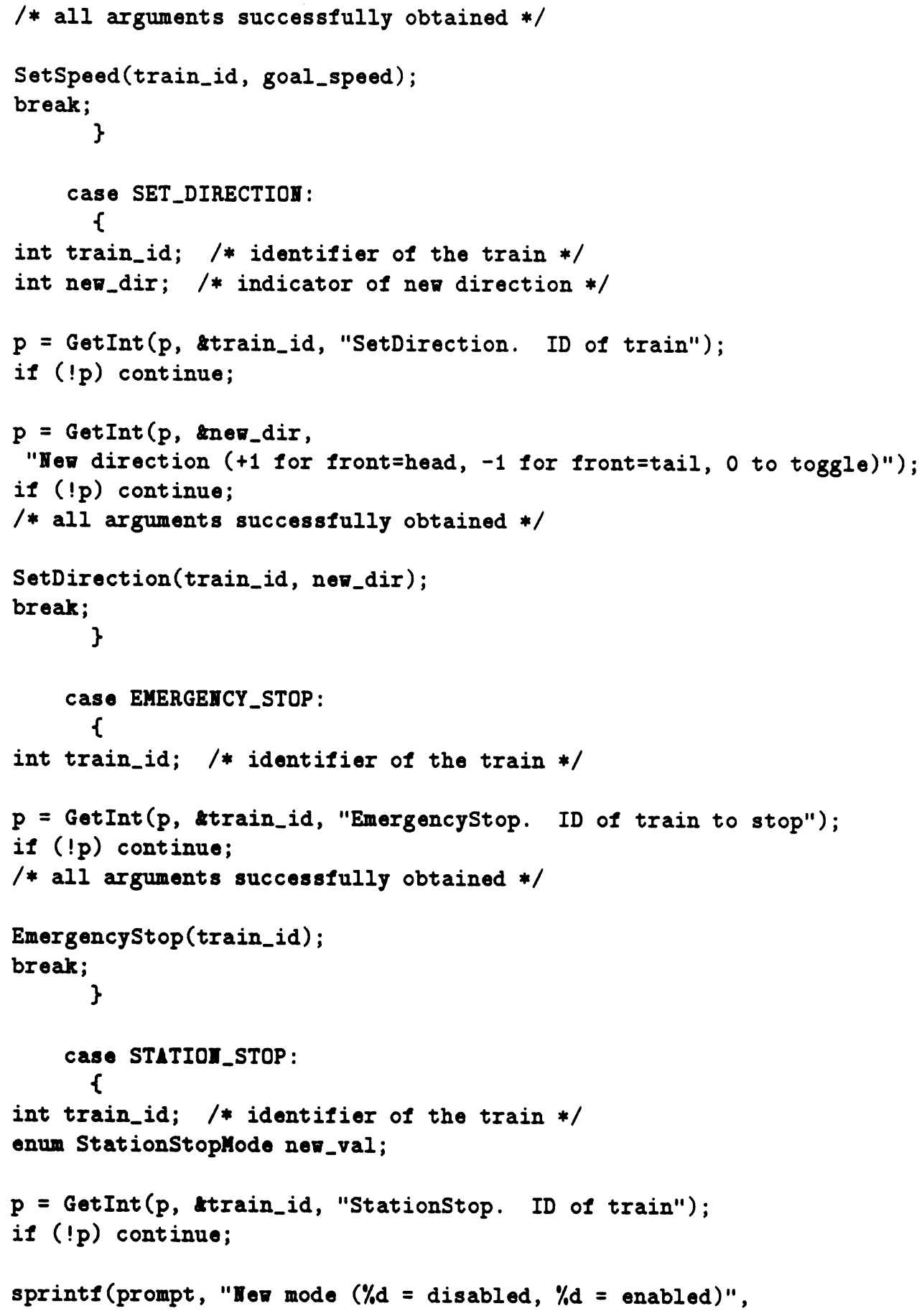




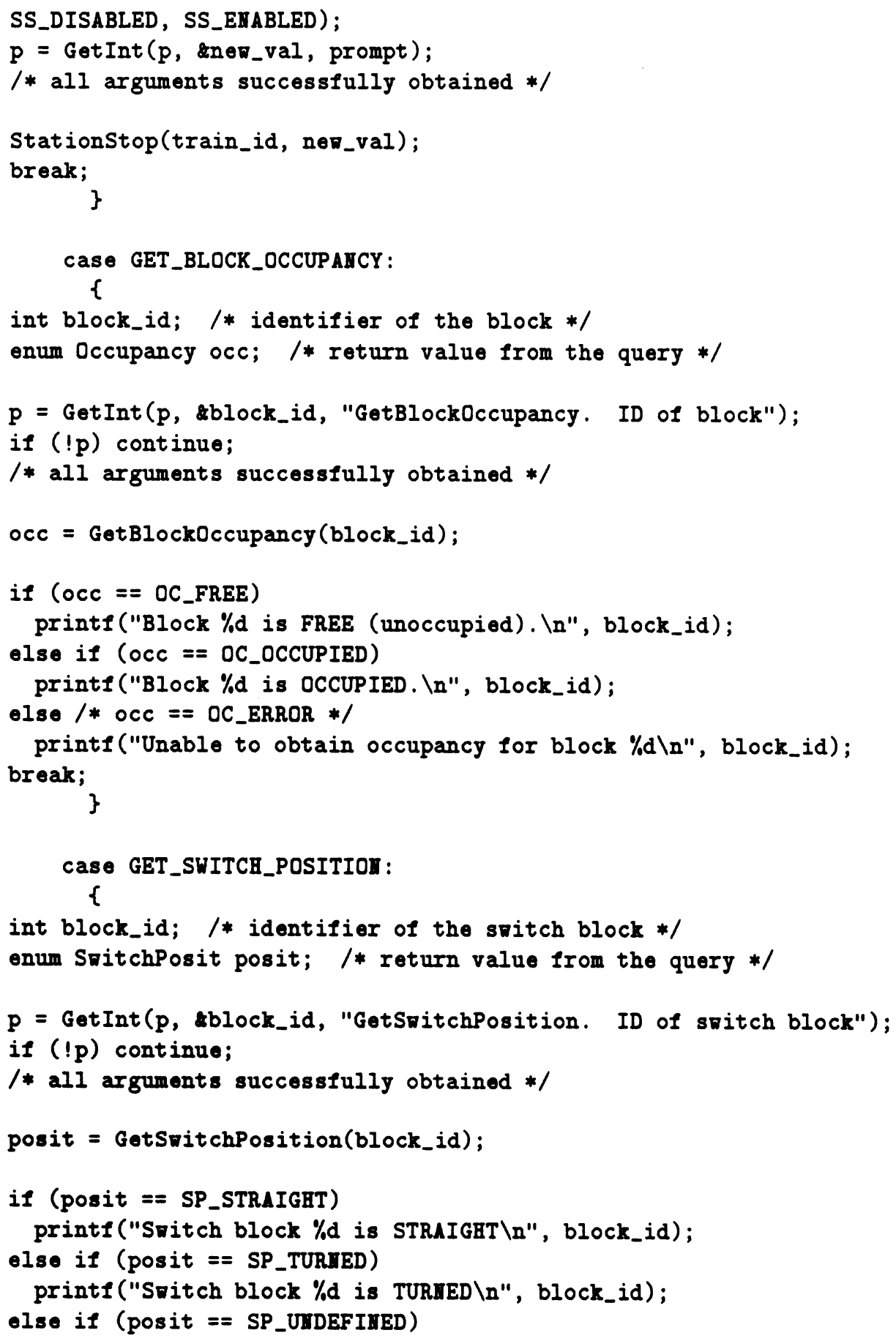


printf("Switch block \%d has UNDEFINED position \n", block_id); else $/ *$ posit $==$ SP_ERROR $* /$

printf("Unable to obtain position of switch block $\% d n$ ", block_id);

break;

\}

case GET_TRAII_STATUS:

\{

int train_id; /* identifier of the train $* /$

enum Trainstatus status; /* return value from the query */

$p=\operatorname{GetInt}(p$, train_id, "GetTrainStatus. ID of train");

if (!p) continue;

/* all arguments successfully obtained */

status = GetTrainStatus(train_id);

if (status $==$ TS_CRASHED)

printf("Train \%d has CRASHED \n", train_id);

else if (status == TS_RUHHIIIG)

printf ("Train \%d is RUHIIMG $\backslash n$ ", train_id);

else /* status $==$ TS_ERROR $* /$

printf ("Unable to obtain status of train $\% d \backslash n "$ ", train_id); break;

\}

case GET_TRAIH_MOTIOH:

\{

int train_id; /* identifier of the train */

enum Trainllotion motion; /* return value from the query */

$p=\operatorname{GetInt}(p$, dtrain_id, "GetTrainMotion. ID of train");

if (!p) continue;

/* all arguments successfully obtained */

motion = GotTrainMotion(train_id);

if (motion $==$ TH_STOPPED)

printf ("Train \%d is STOPPED $\backslash n$ ", train_id);

else if (motion $==$ TM_MOVIIG)

printf ("Train \%d is MOVIMG $\backslash n$ ", train_id);

else /* motion $==$ TM_ERROR $* /$

printf("Unable to obtain motion information for train $\% d \backslash n$ ", 


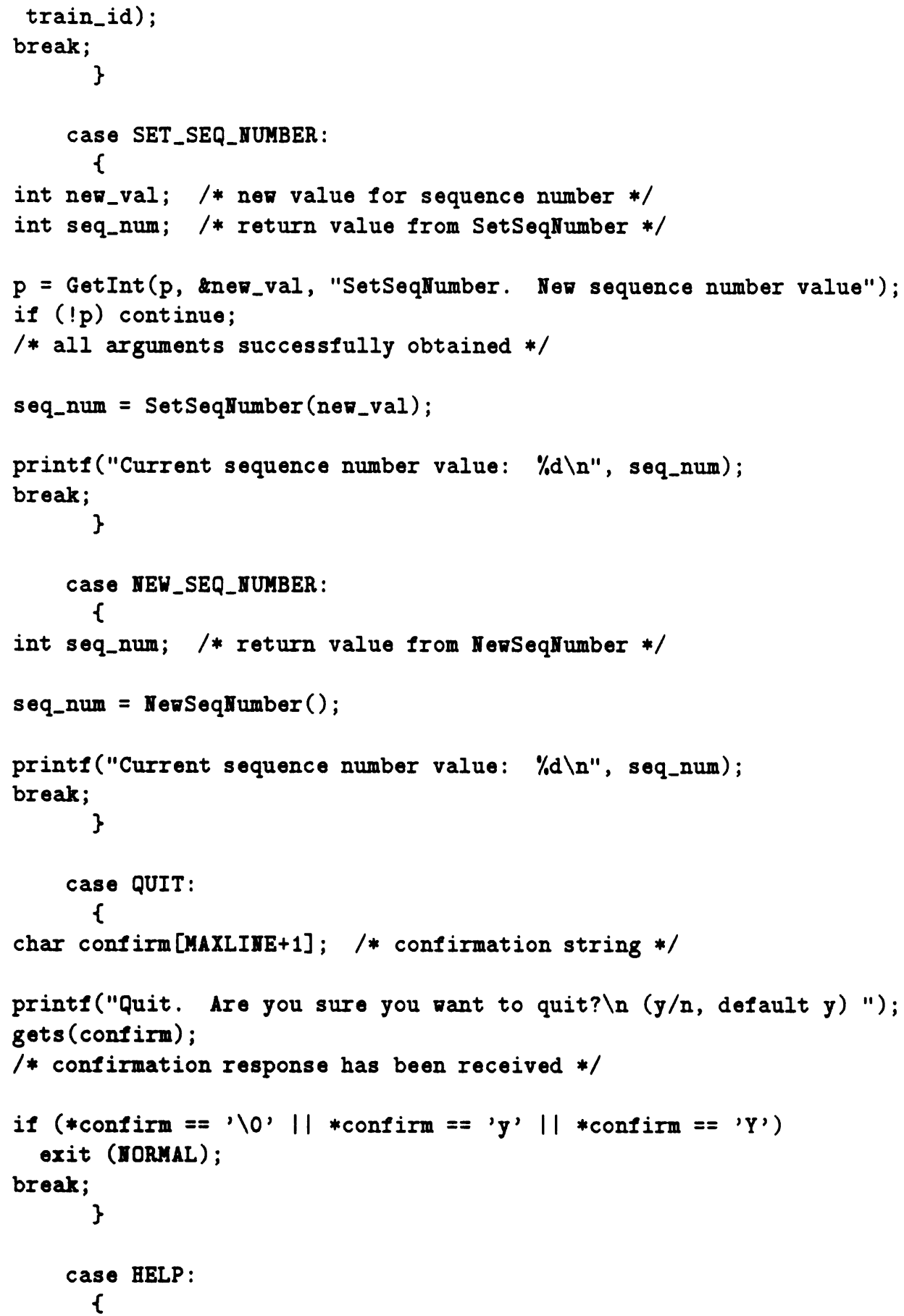




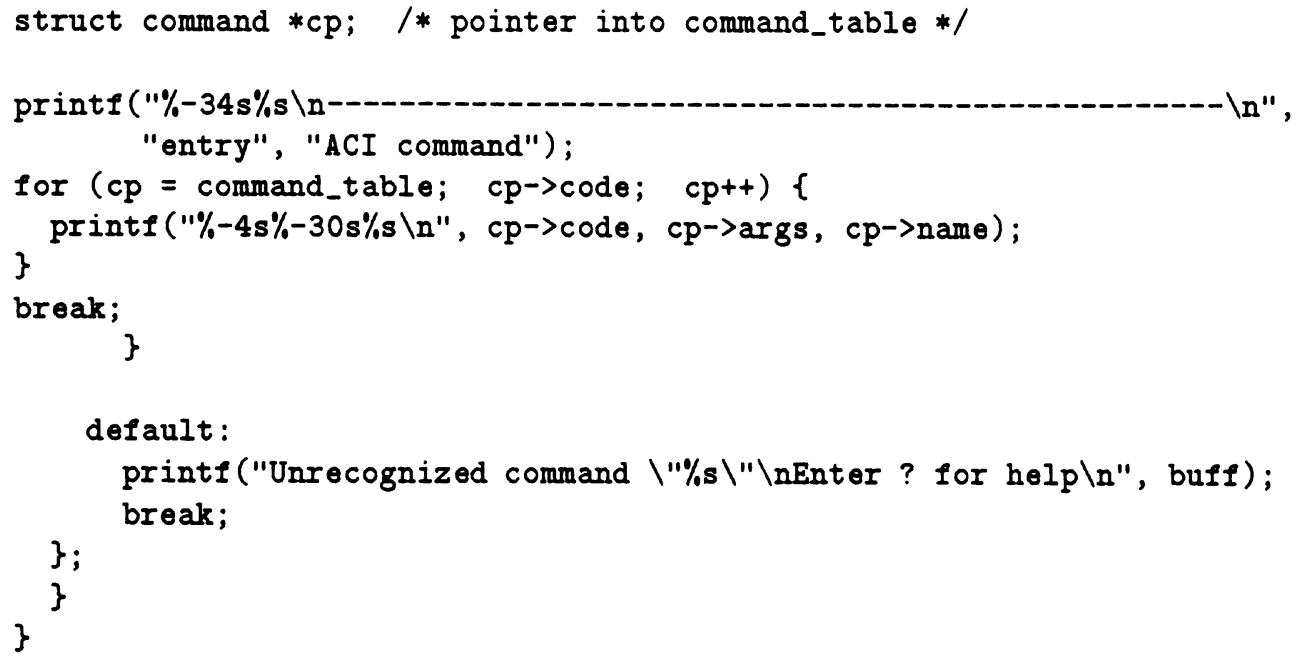




\title{
Appendix C
}

\section{Code Listings: LLI}

\author{
C.1 cpi.h, Interface Header File
}

/* \$RCSfile: cpi.h,v \$ \$Revision: 1.65 \$ \$Date: 92/11/24 14:41:00\$*/

/* cpi.h -- cpi message formats. */

/* This file automatically generated by m4 from cpi.hm */

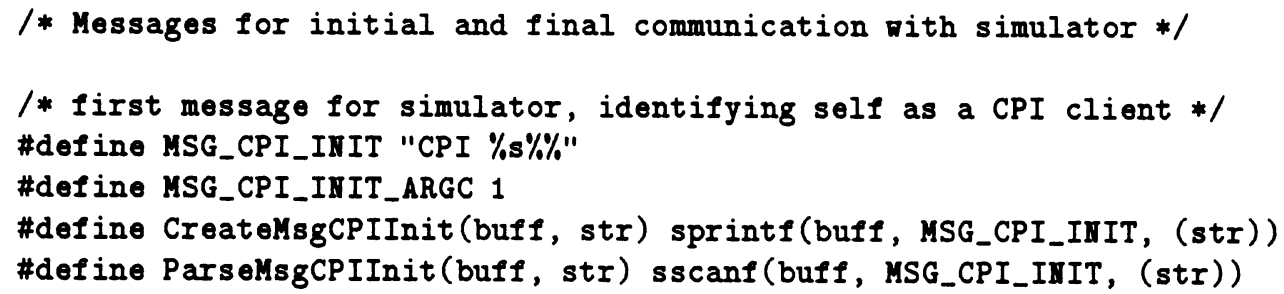


\#define ParseMsgQuitAll(buff, str) sscanf(buff, MSG_QUIT_ALL, (str))

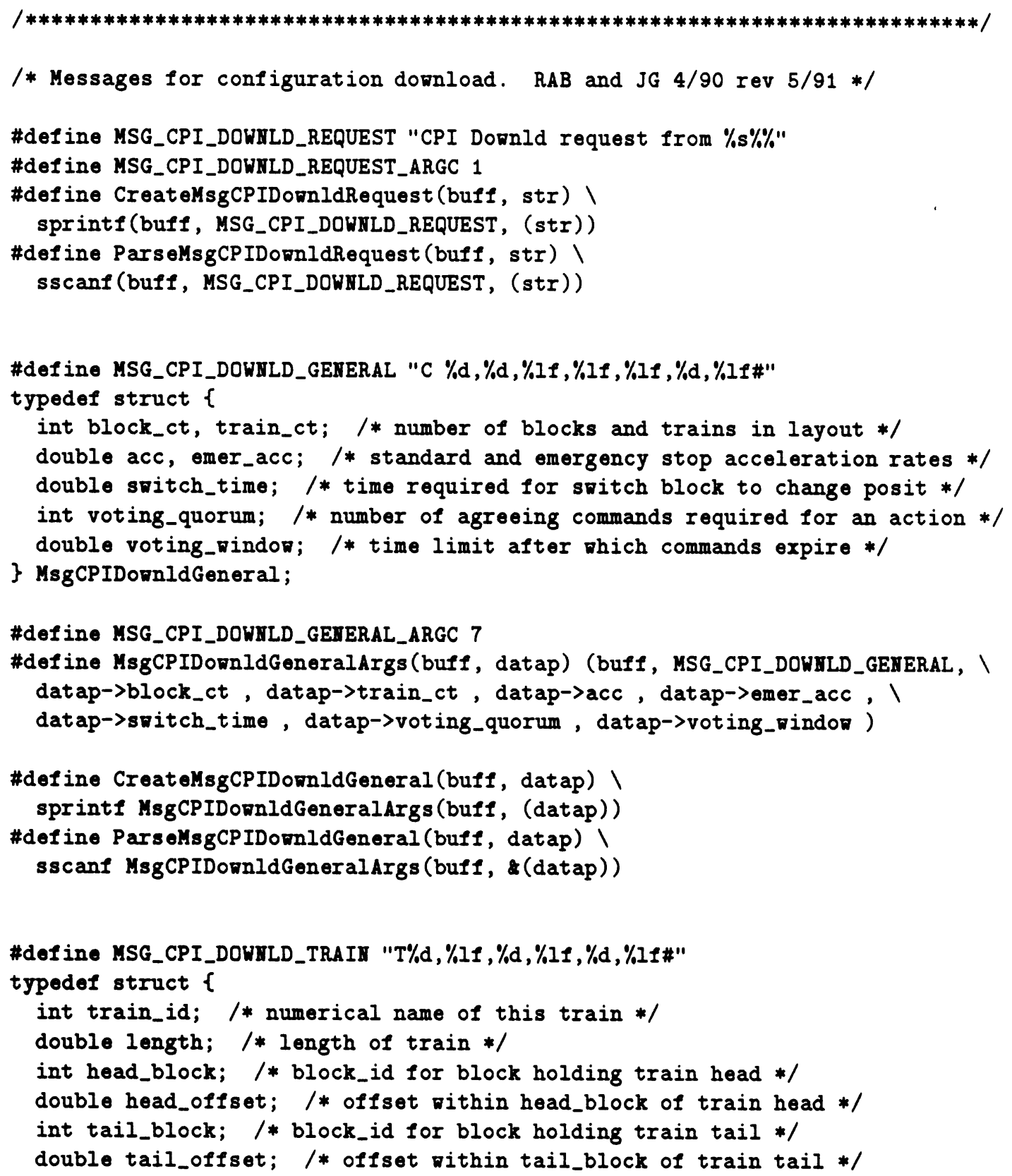




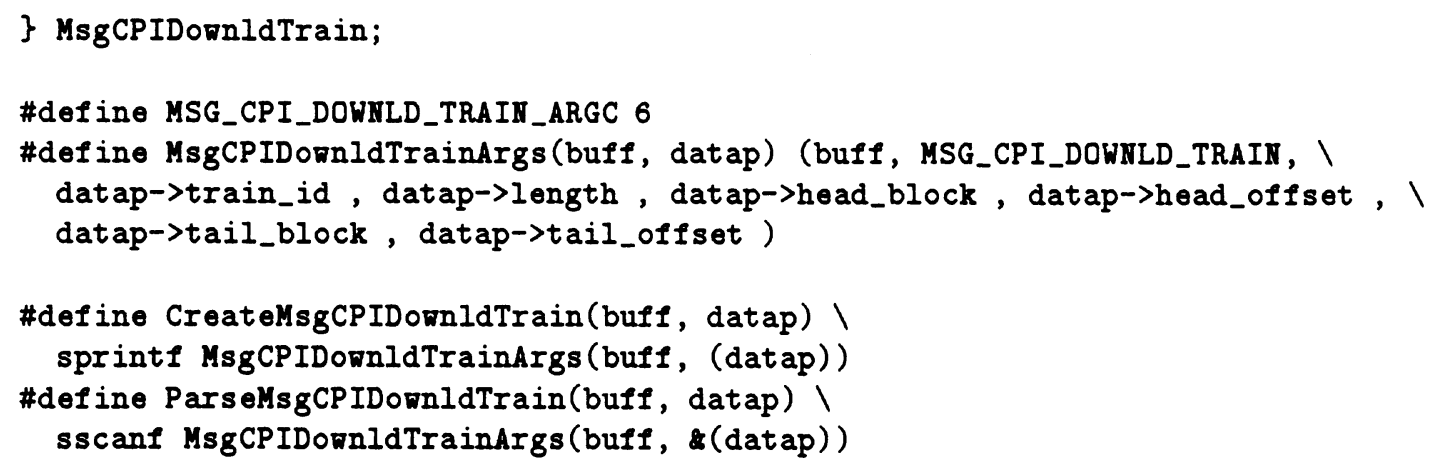




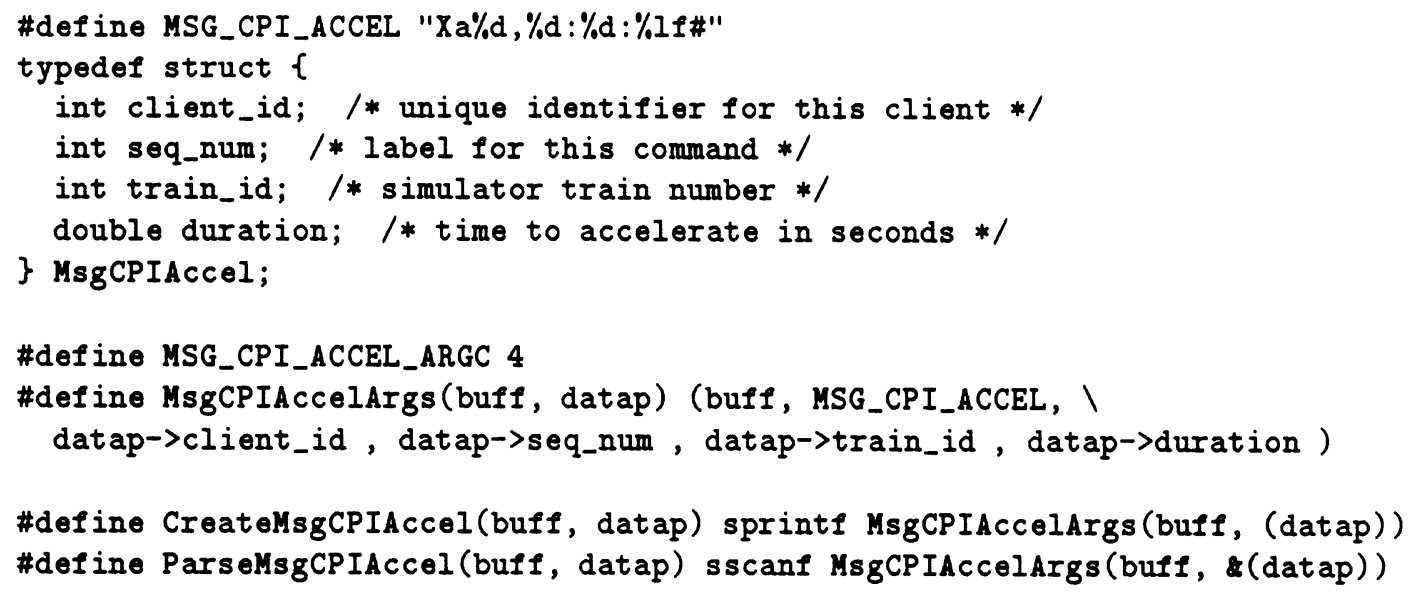




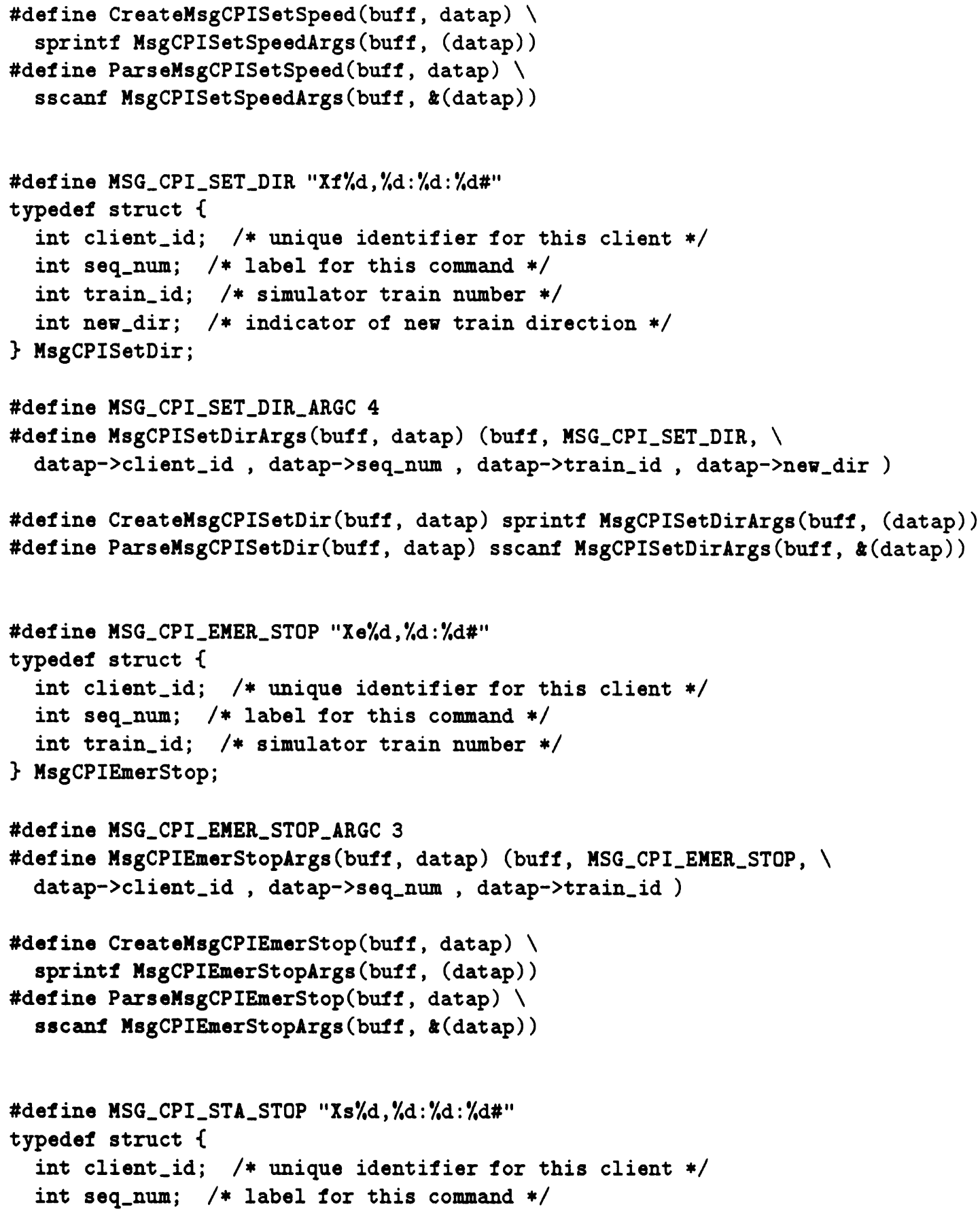




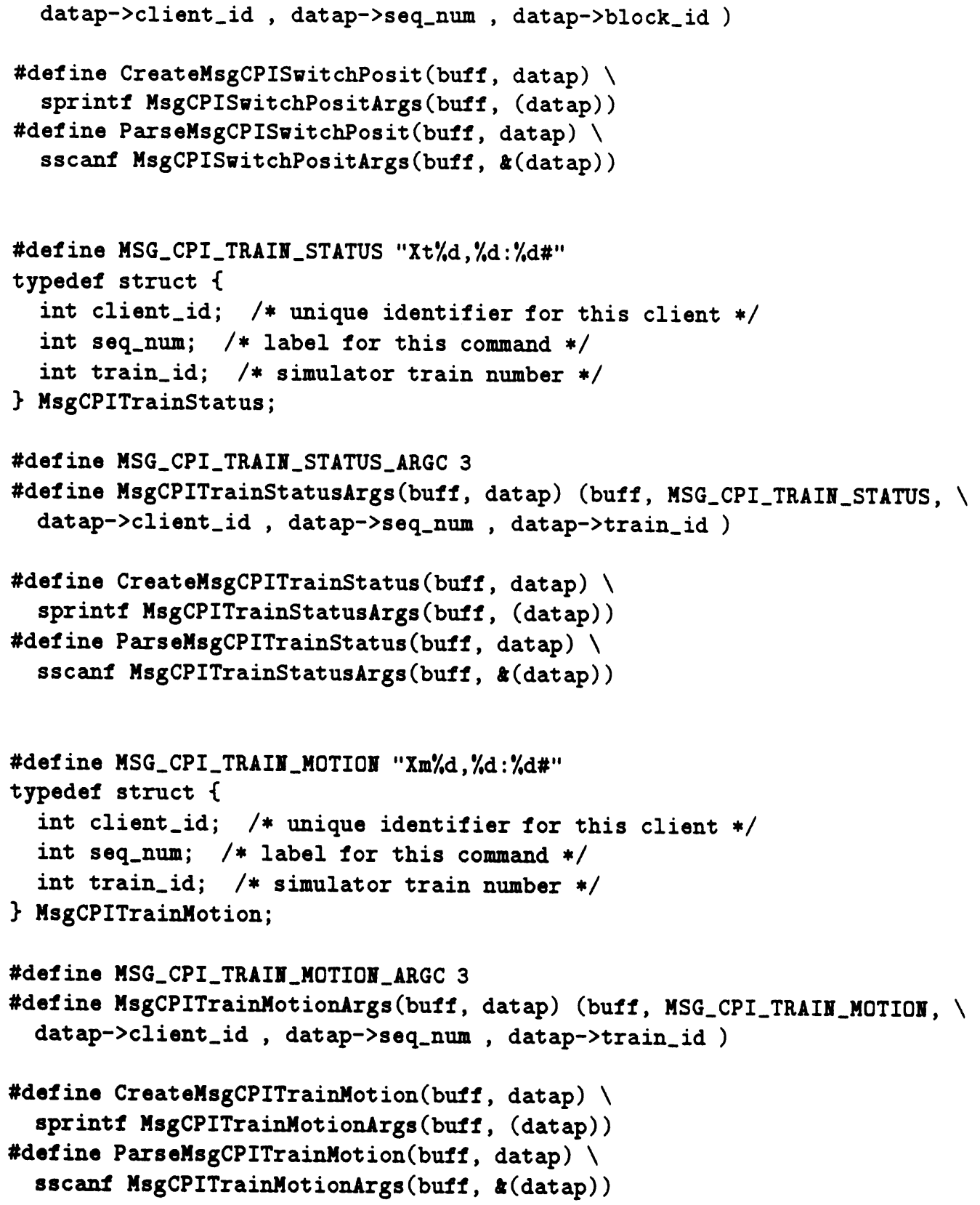




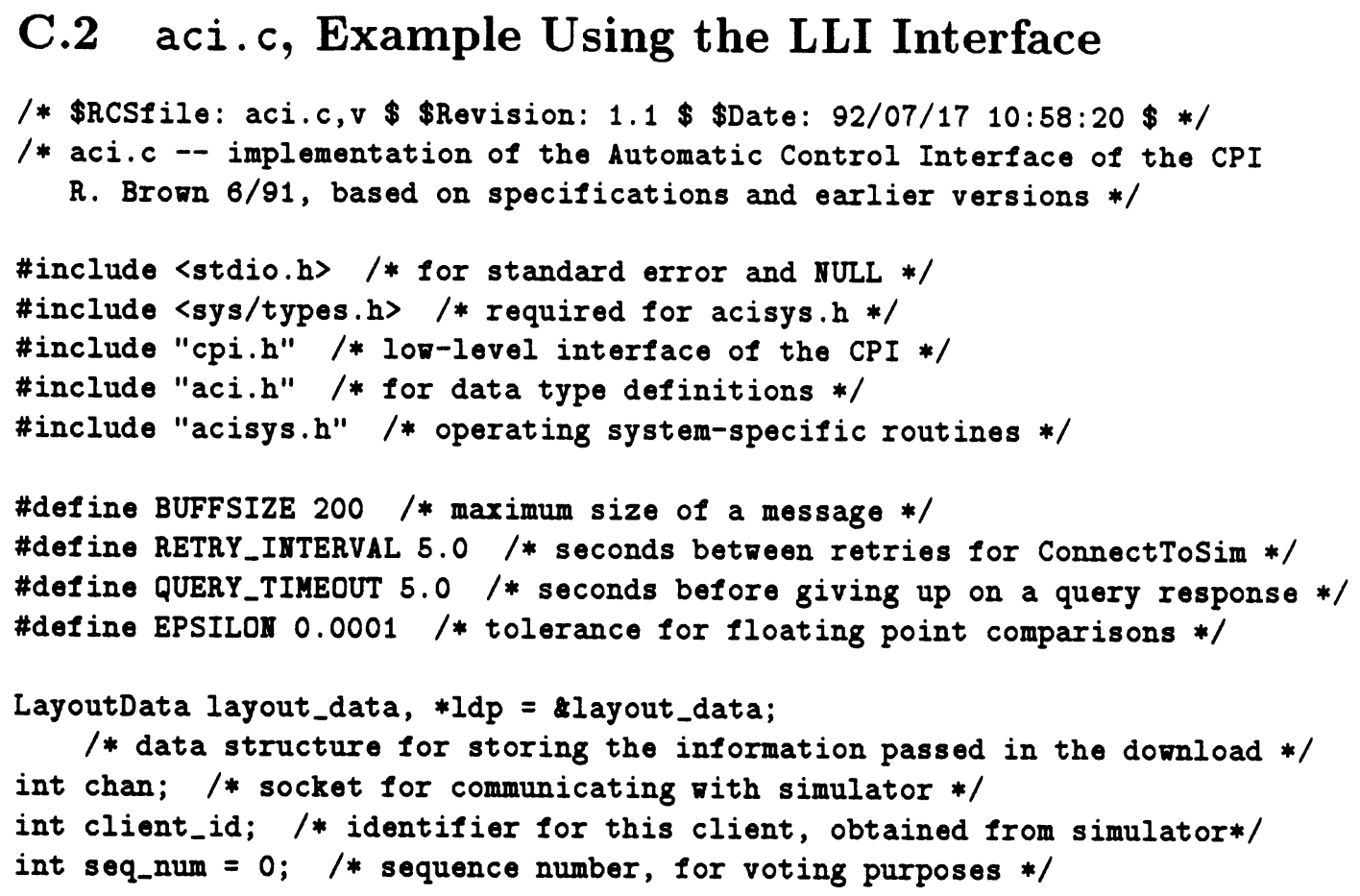

$/ * * * * * * * * * * * * * * * * * * * * * * * * * * * * * * * * * * * * * * * * * * * * * * * * * * * * * * * * * * * * * * * * * * * * * * * * * * * * /$

1* SendinitMessage sends the simulator the initialization message for this application. Returns nonzero on success, zero on failure. 


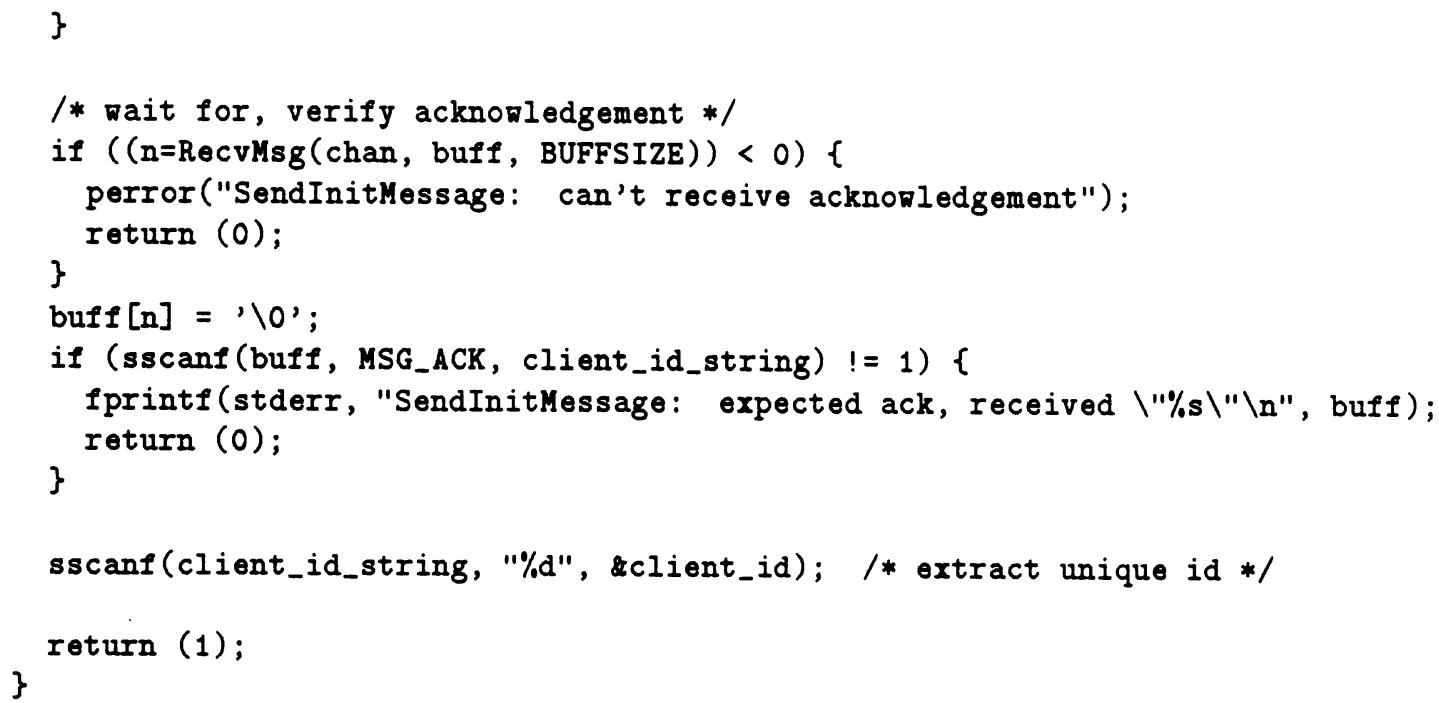




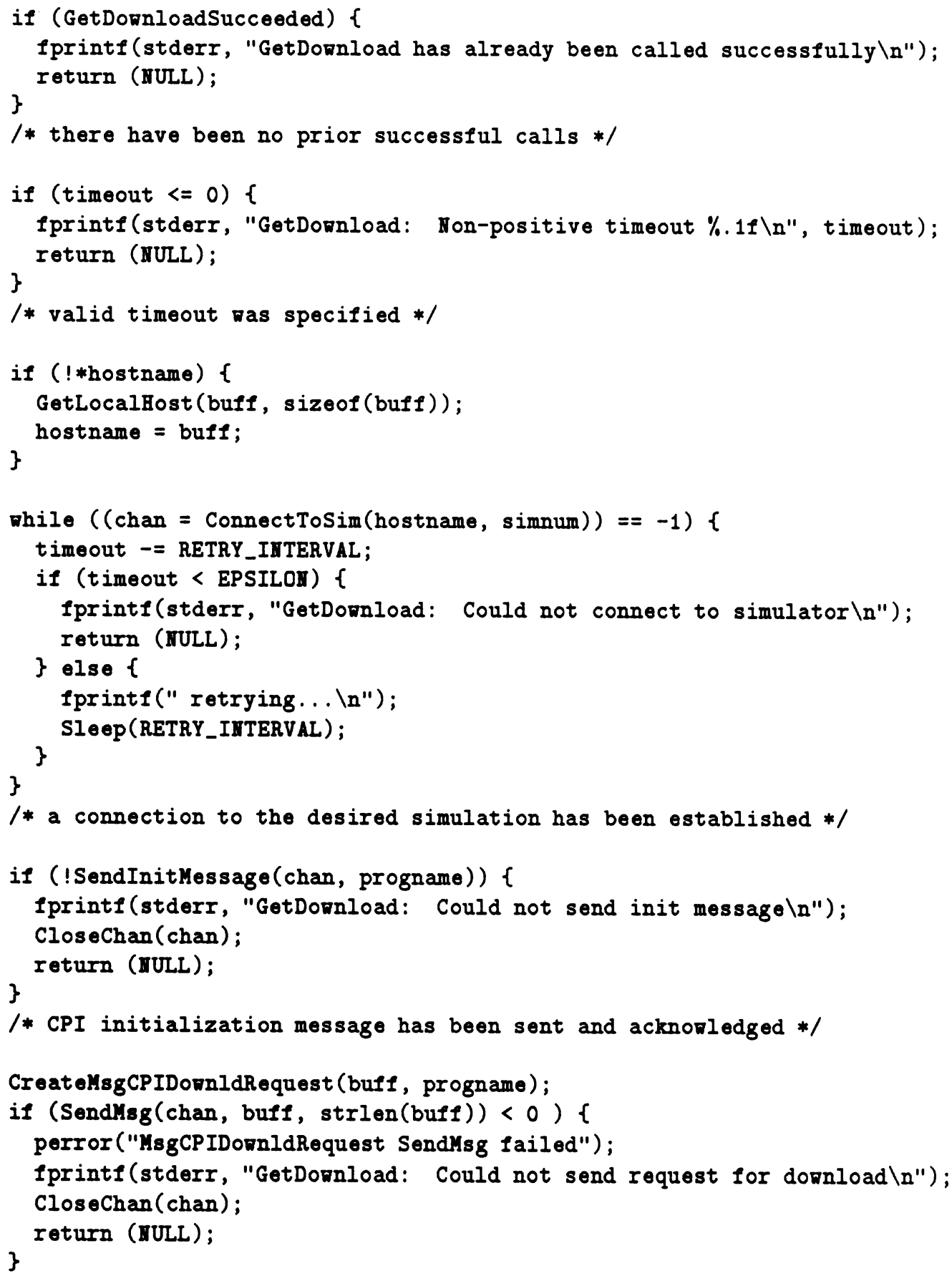




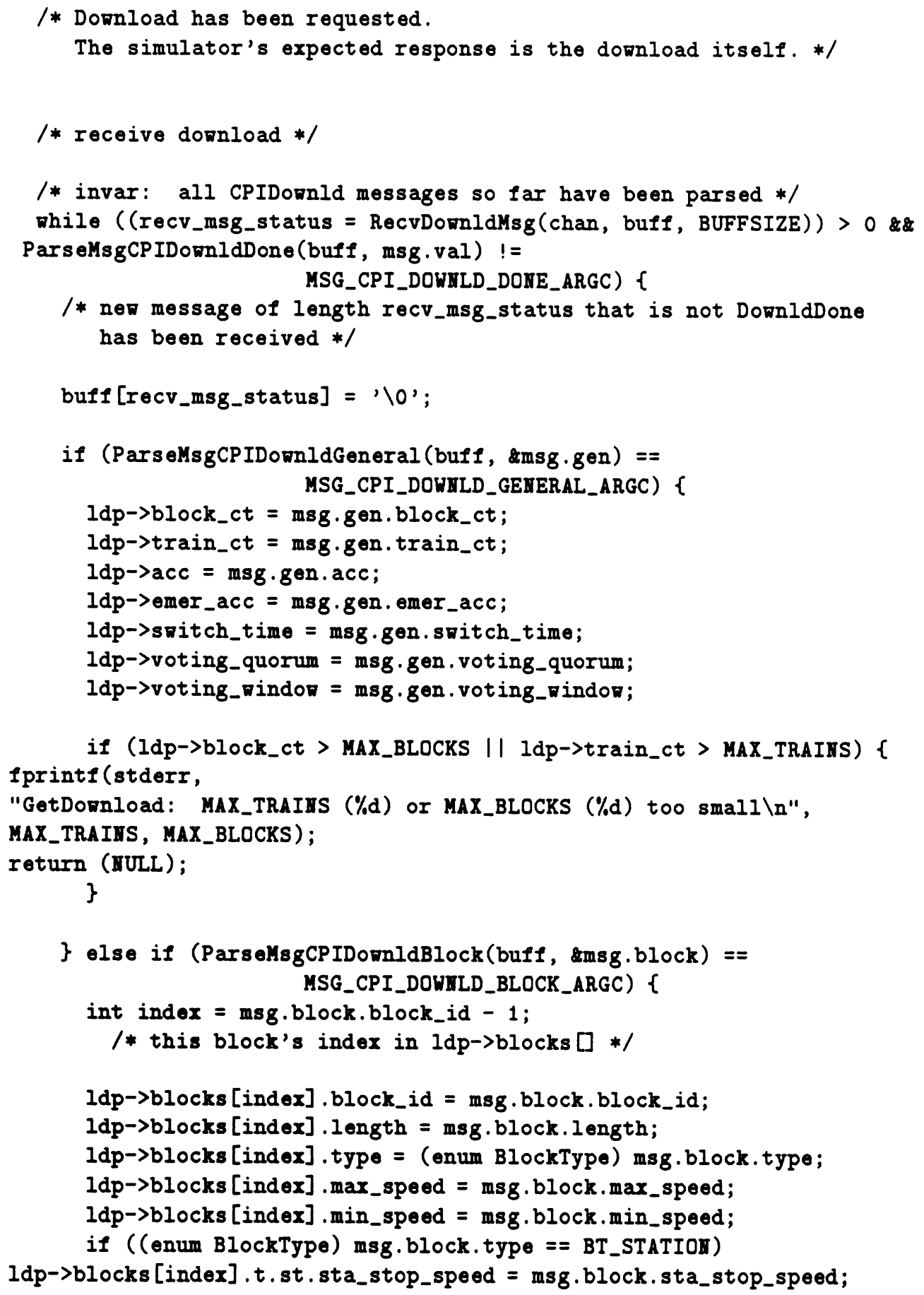


if ((enum BlockType) msg.block.type $==$ BT_CROSS)

ldp->blocks [index].t.cr.cross_id = msg.block.cross_id;

\} else if (ParselsgCPIDownldLink (buff, \&msg.link) == MSG_CPI_DOWHLD_LINK_ARGC) \{

int index $=$ msg.link.block_id -1 ;

/* this block's index in Idp->blocks [] */

switch (ldp->blocks [index].type) \{

case BT_REGULAR:

case BT_STATIOI :

case BT_CROSS :

ldp->blocks[index].t.Ig.tail = msg.link.tail;

ldp->blocks [index] .t.rg.head =msg.link.head1;

break;

case BT_JOII :

ldp->blocks [index] .t.jn.tail = msg.link.tail;

ldp->blocks [index] .t. jn. head1 = msg.link.head1;

ldp->blocks [index] .t. jn. head2 = msg.link.head2;

break;

case BT_SWITCH :

ldp->blocks [index].t.sw.tail = msg.link.tail;

ldp->blocks [index].t.sw.straight = msg.link.head1;

ldp->blocks[index].t.sw.turn = msg.link.head2;

break;

\}

\} else if (ParseMsgCPIDownldTrain(buft, emsg.train) == HSG_CPI_DOWHLD_TRAII_ARGC) \{

int index $=$ msg.block.block_id -1 ;

/* this train's index in ldp->trains $[* /$

1dp->trains [index] .train_id = msg.train.train_id;

ldp->trains [index] . length = msg.train.length;

ldp->trains [index].head.block = msg.train.head_block;

ldp->trains [index] .head.offset = msg.train.head_offset;

ldp->trains[index].tail.block = msg.train.tail_block;

ldp->trains[index] .tail.offset = msg.train.tail_offset;

\} else $\{/ *$ unrecognized message format */

fprintf(stderr, "GetDownload: Unrecognized message from simulator $\backslash n$ "); fprintf (stderr, "\%s\n", buff);

\} return (IULL); 


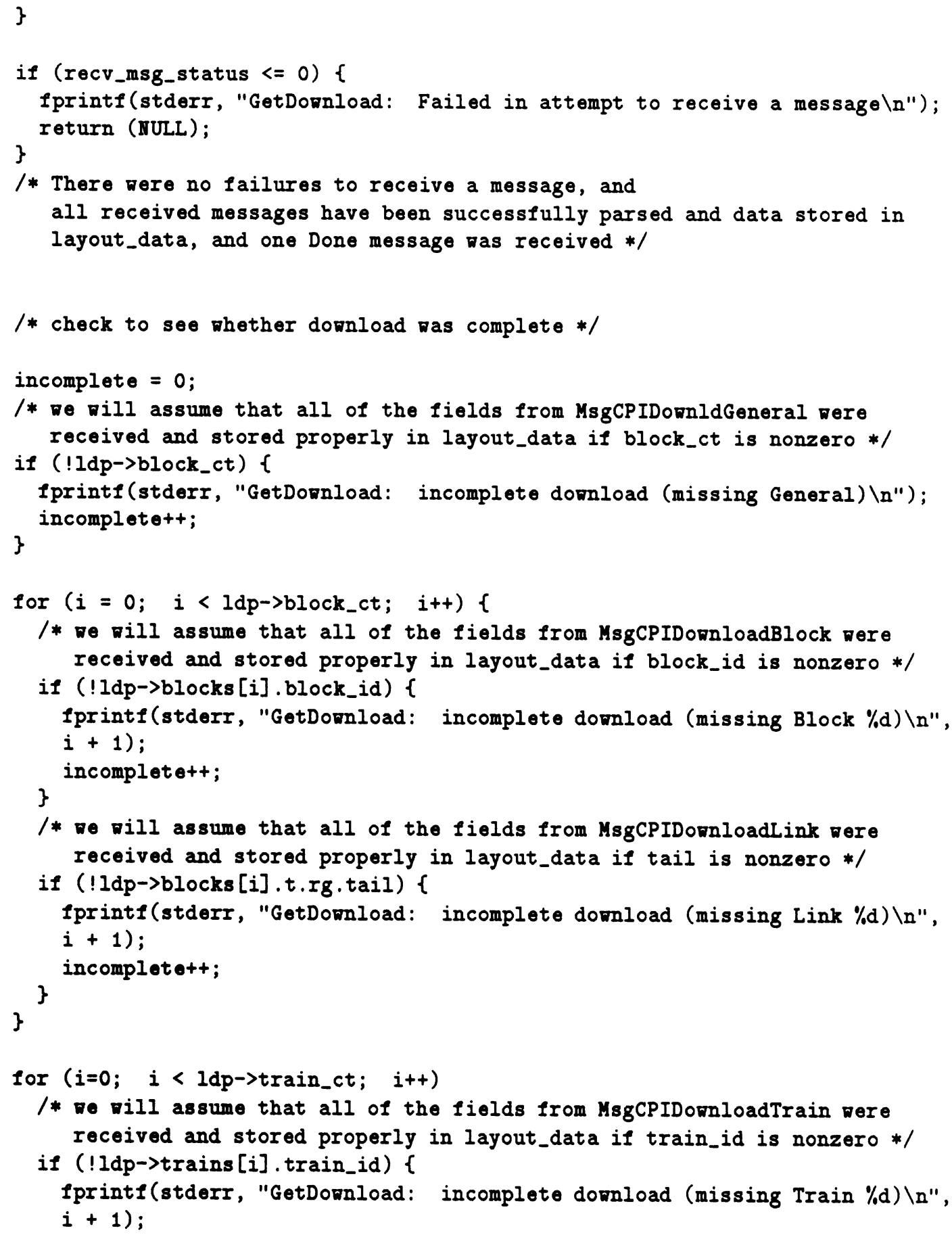




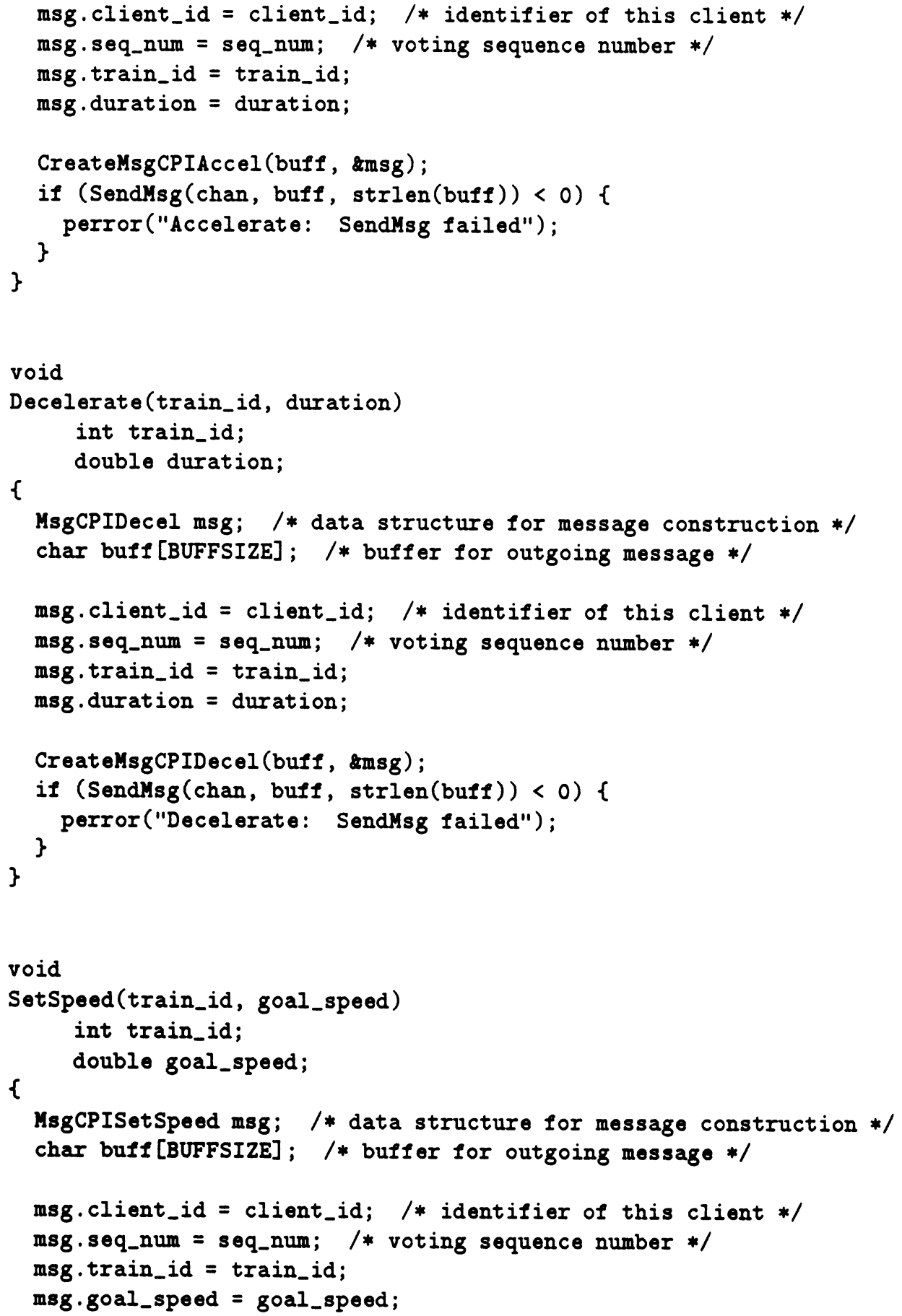




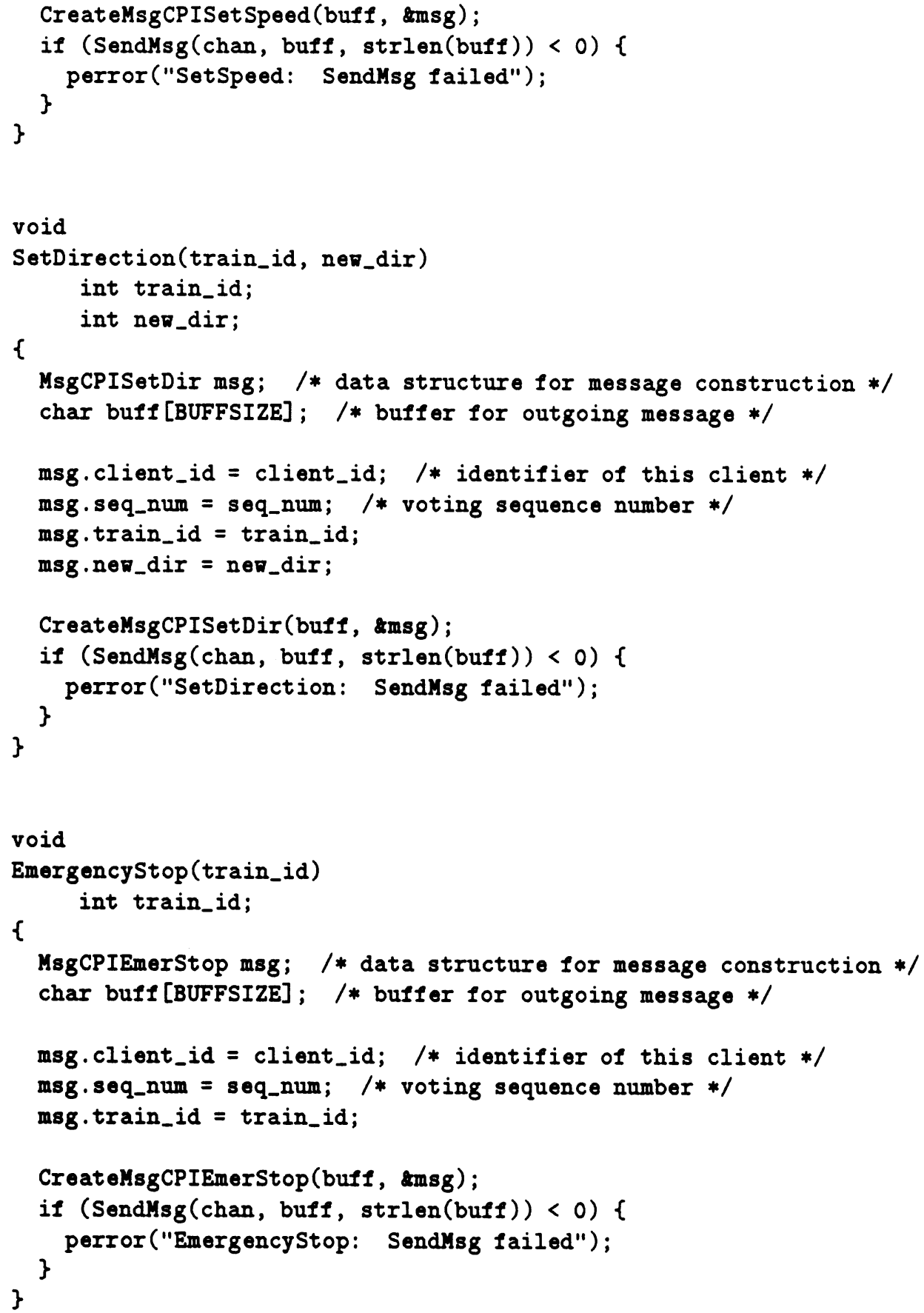




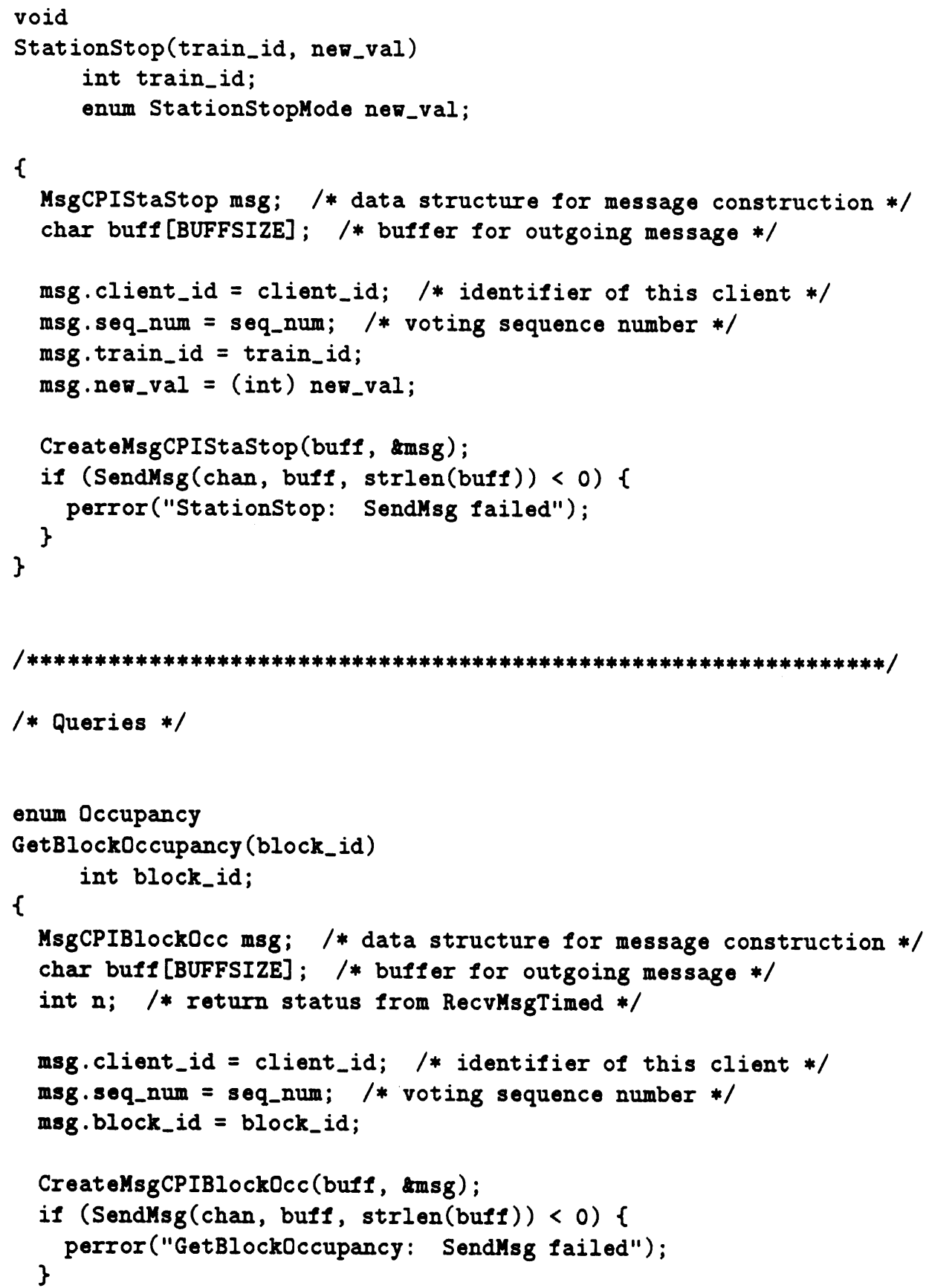




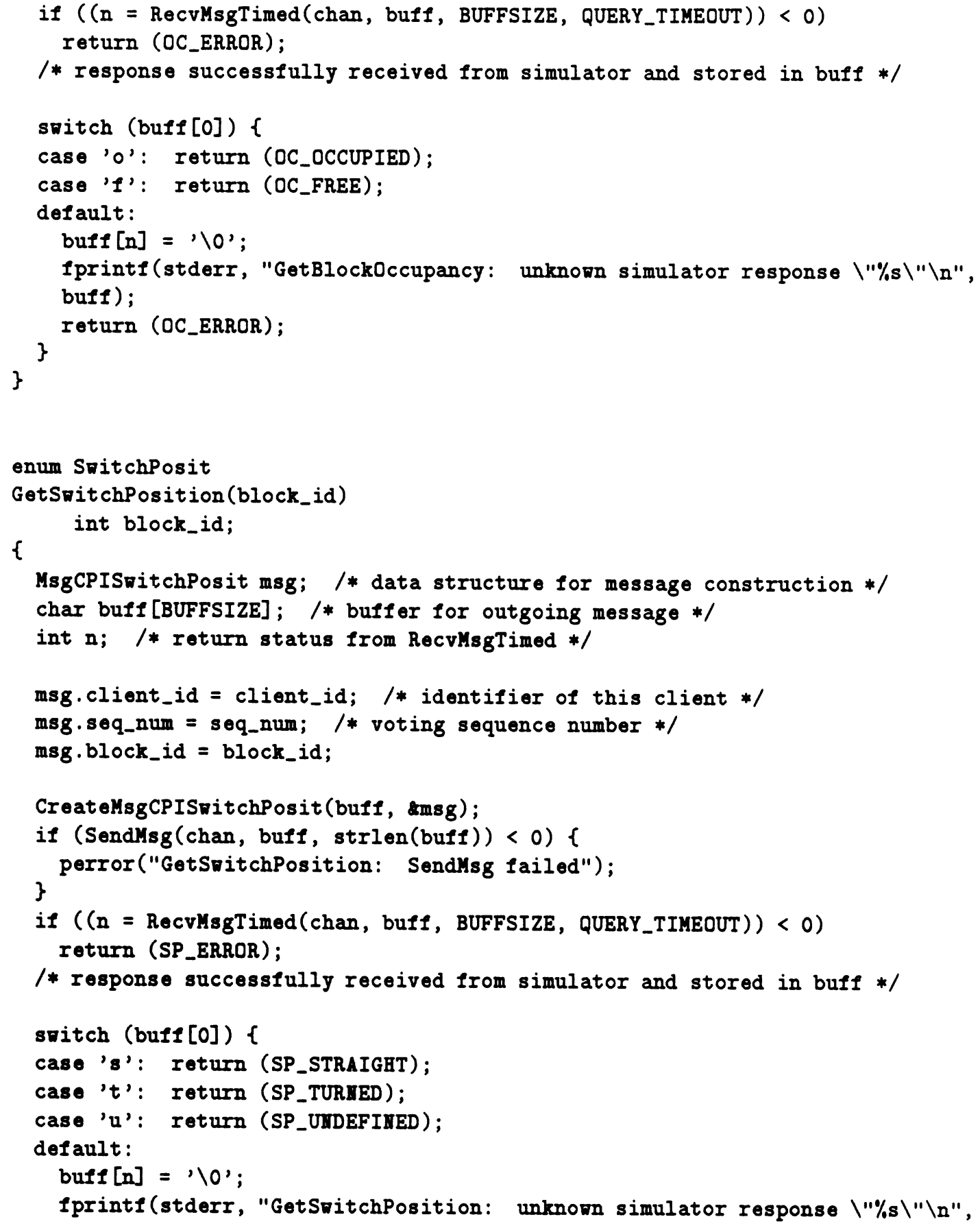




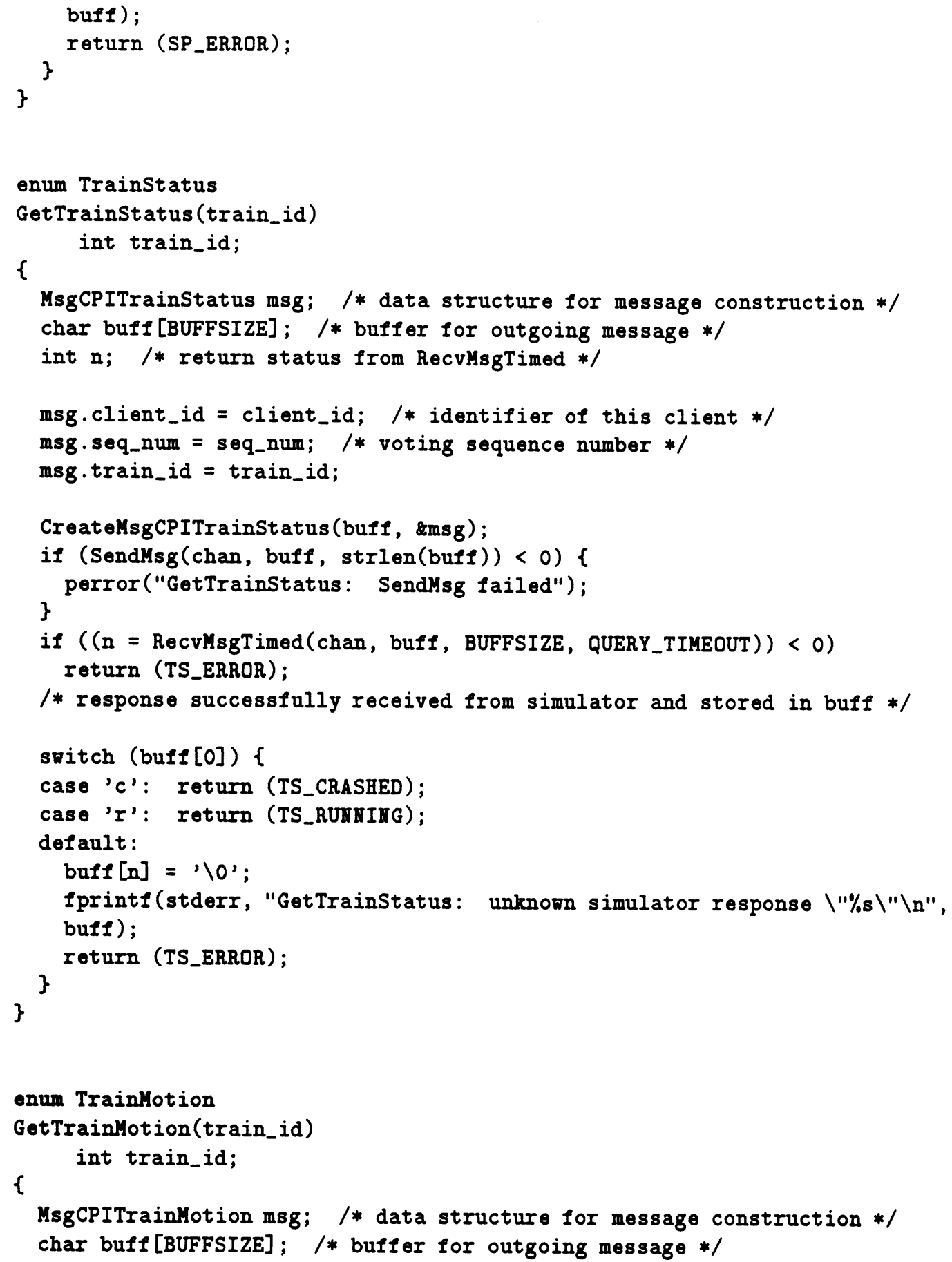




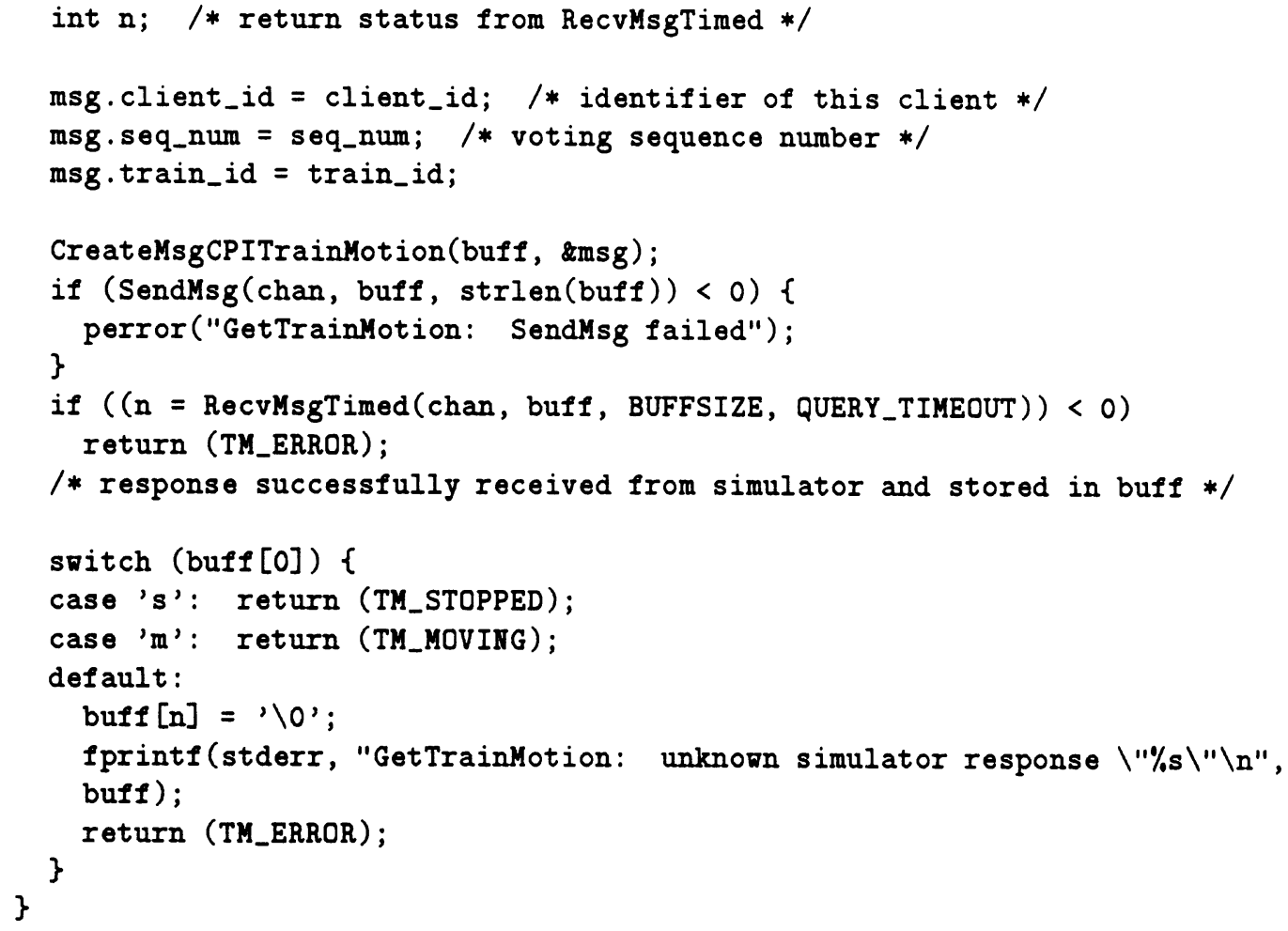

int 


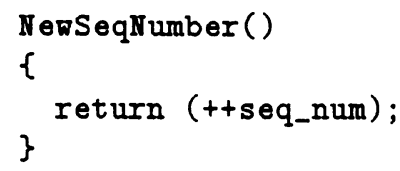




\section{Appendix D}

\section{Reference Pages}

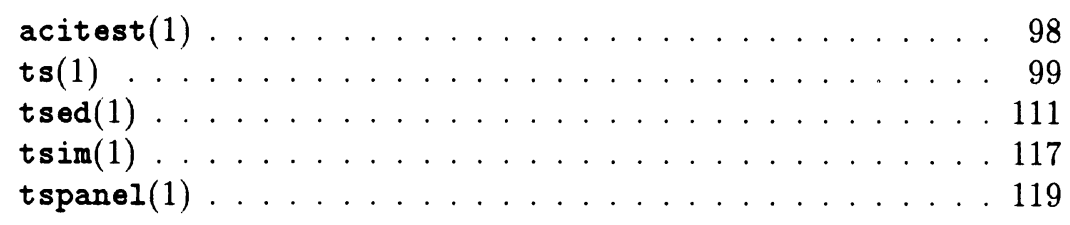


acitest(1)

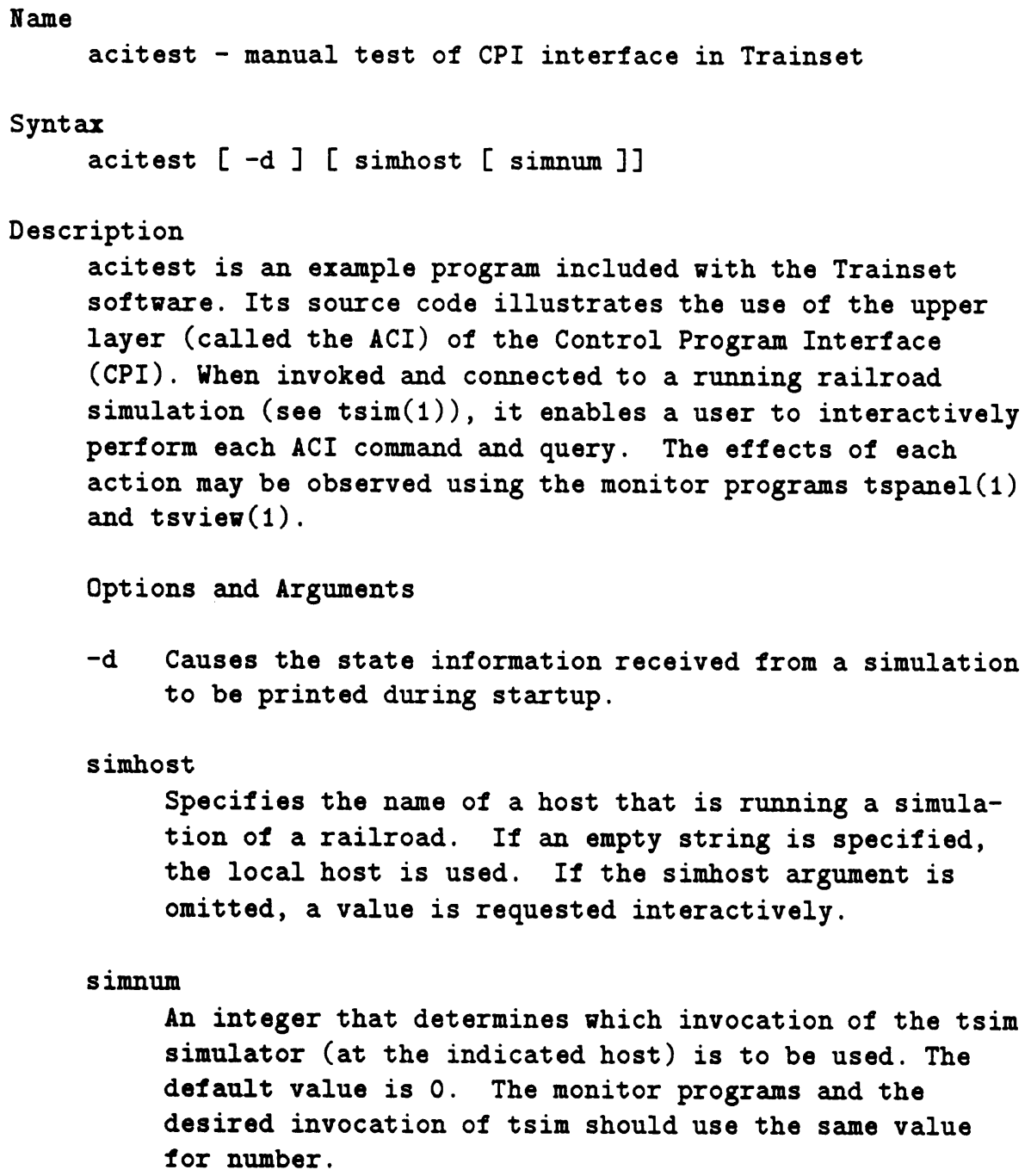


Name

ts - launch Trainset applications

Syntax

ts [ flags...]

ts -edit [ file] [ -layout file ] [-tsroot dir ] [ X11options ]

Description

ts invokes ("launches') applications related to the Trainset railroad simulation softrare in a computing environment that may involve a heterogeneous network of computing systems. With no options, ts typically starts a demonstration consisting of a simulation of a sample railroad, an X11 application that graphically displays the current state of the railroad, and another $X 11$ application that provides for manual control of the railroad. ts options enable the user to select which applications are to be launched, choose the railroad layout to be simulated, specify the host to launch from, etc.

Programmers may write software that interacts with Trainset, including programs that automatically control a railroad and custom versions of the basic Trainset softrare. ts provides a configuration mechanism for adding launch information describing such programs to its database.

When invoked with -edit as the first command-line option, ts invokes the tsed editor for railroad layouts and launches no other applications. The options -tsroot and -layout are recognized by $t s$ in the case of -edit, and may appear either in an environment variable TSOPTS or on the command line. file names the layout data file to be edited. file may be presented as the second command line argument if it does not begin with a dash ' - '. It may also be provided using the -layout option. -tsroot specifies the tsed search paths for layout data files, as discussed in Search Paths belor. In addition, any additional options on the command line, in 
$\operatorname{ts}(1)$

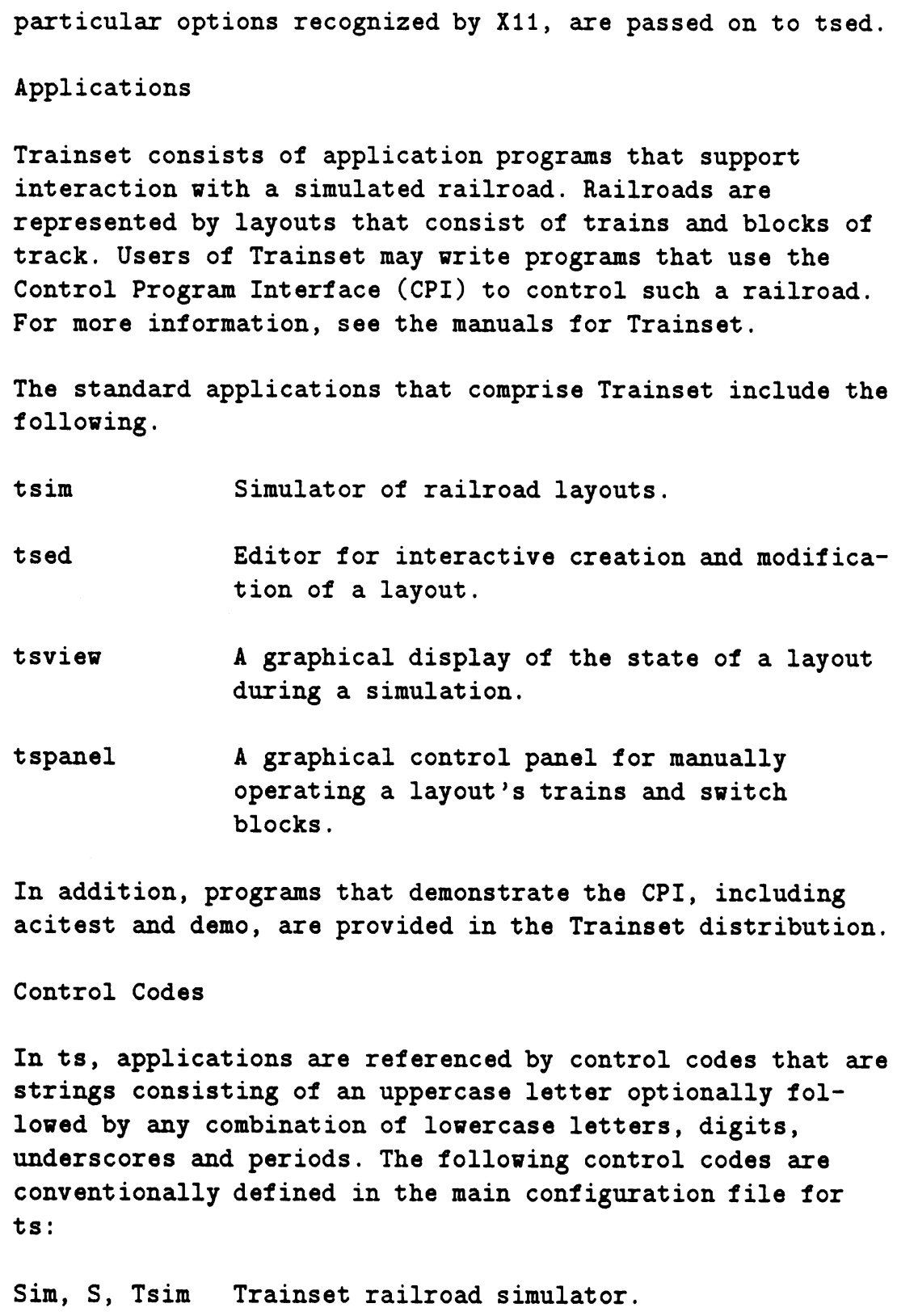


Panel, P, Tspanel

Control panel application.

Viever, V, Tsview

Viewer application for observing a simulation.

Acitest, A Acitest program that allows manual experimentation with CPI interface features.

Demo, D Demonstration of the CPI interface discussed in the Trainset manual.

Any of the ts options -args, -delay, -direct, -display, -host, -tsroot, -xterm and -xtermargs can be localized to apply to a specific application by prefixing that option with the appropriate control code(s). For example, either 'Sim:-host loki' or 'S:-host loki' specifies that the simulator should be launched at the site loki. Also, 'PV:-display thor:0.0' causes the panel and viever applications to display graphics output on the $X 11$ server thor:0.0. Arbitrary arguments can be passed to applications using the -args option prefixed by the appropriate control code, e.g., 'Myprog:-args "-level 4 -trace"'. Quoting (e.g., using '"') enables spaces to be included in the argument string.

If the colon that terminates a prefix is followed immediately by a character ( 0.8 ., dash ' - ') that cannot be part of a control code name, then that colon may be omitted. For example, 'Sim-host loki' is equivalent to 'Sim:-host loki'.

An alias facility is provided for associating multiple control codes with a single application. Aliases may be declared in configuration files (discussed below) or by using the -alias option. The alias feature can be used to change the associations betreen control codes and applications. For example, suppose that a control code Train_control_2 is associated with an application that has 


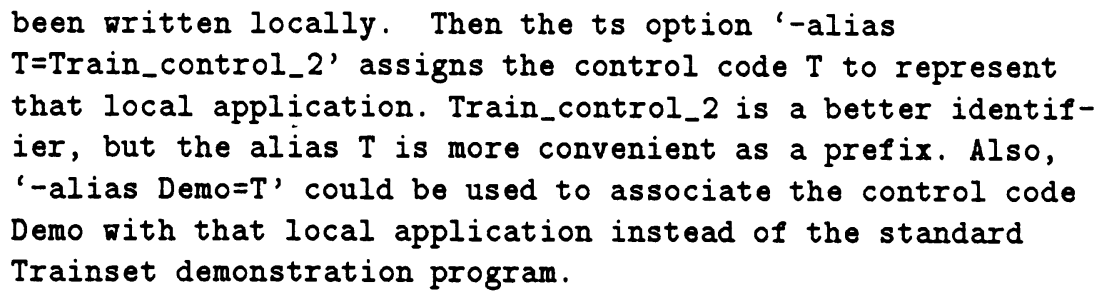

The -dup option may be used to invoke multiple copies of an application. This feature supports the launching of replicated programs. -dup clones the launch information gathered so far for the application in question and associates a control code with the copy. The launch attributes in the two copies may thereafter be modified independently.

\section{Search Paths}

The directory tsroot identifies the location of the Trainset installation in the local file system. Tsroot may be specified as the value of an environment variable TSROOT or on the command line using the -tsroot option.

Ts searches for its own configuration files in the directories.,./lib, tsroot and tsroot/lib (in that order).

Layout data files for a simulation are sought in the directories.,./layouts, tsroot, tsroot/layouts and

tsroot/trainset/layouts. The default extension '.I' is appended (if omitted) to a layout data file name by Trainset applications and searching is repeated if a file with the given name was not found. If no layout data file is specified, the file tsroot/layouts/sample.l is used.

Binaries for Trainset applications are sought using the environment variable PATH as usual.

Launching Applications

Unless the -edit option is specified, ts gathers information 
from the following sources, in order, before launching applications.

Main configuration file

This file associates control codes with applications, contains invocation information for applications (e.g., name of executable and default arguments) and provides a default list of applications to be launched. See ts.config(5) for the file format. The name of the main configuration file may be specified with the -config option. The default main configuration file is tsroot/ts.config.

Auxiliary configuration files

One or more additional configuration files may be designated to supplement the main configuration file. These are specified by the ts options -confign where $n$ is a single digit. Auxiliary configuration files are processed in increasing order of $n$. The values of $n$ need not be contiguous. Auxiliary configuration files enable individual users and groups of users to install their own applications and customize launching information for applications that appear in the main configuration file. Attributes for the launch are either overridden or appended as appropriate. If a non-empty list of applications to be launched is included in an auxiliary configuration file, it overrides any prior list. There are no default auxiliary configuration files.

Environment variables TSROOT, TSOPTS and DISPLAY If there is an environment variable TSROOT, it is treated as the default value for tsroot (see Search Paths above). If there is an environment variable TSOPTS, it is treated as a list of ts options that are parsed prior to the options specified on the command line. If there is an environment variable DISPLAY with value X11-display, then the option -xtermargs X11display is implicitly processed just prior to any 
$\operatorname{ts}(1)$

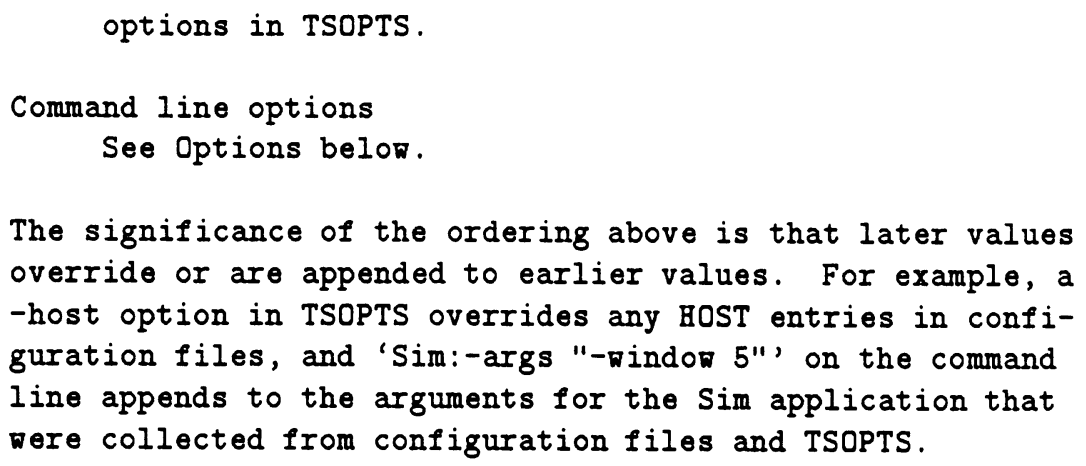


options . )

ts provides two methods for launching applications.

xterm Method

If the configuration file attribute XTERM for an application is a string of positive length or if the -xterm option is used, then the application is invoked in an xterm(1) window that is created for that purpose. That application's standard input and output will be associated with the xterm window. Arguments can be passed to xterm using the -xtermargs option discussed below.

Direct Method

Applications that are launched without using the xterm method are invoked asynchronously by the ts process, and share that process's standard output. The standard input for all such applications is /dev/null, except the last application launched by the direct method inherits the ts process's standard input as well as standard output. Thus, an interactive application (e.g., acitest(1)) may be launched using the direct method provided that it the last such application specified. See the Examples section.

xterm is the default launch method. The direct method is currently implemented for the local host only, i.e., the host at which the ts command was entered. Thus, any -host options are ignored for applications launched by the direct method.

Options

-alias control1=control2

Declares the control code controll to be an alias for the control code control2. The two codes may then be used interchangeably for referring to the application launch attributes associated with control2, if there is such an application. The symbol ' $=$ ' is optional. 
-dup control1=control2

Make a copy controll of the application launch attributes associated with control2. Since the two control codes represent different copies, a change in the attributes referenced by controll will have no effect on the attributes referenced by control2. This is useful for invoking multiple copies of the same application, e.g., parallel copies of the same CPI program for controlling a railroad. The symbol ' $=$ ' is optional.

-args arglist

Provides program-specific arguments to be passed to applications. This option is ordinarily prefixed by control codes, e.g., 'Sim:-args "-update 0.5 "'.

-config filename

Specifies the name of the main configuration file.

-confign filename

Specifies the name of the nth auxiliary configuration file.

-delay seconds

Specifies an integer number of seconds to pause just after launching each application.

-direct

Specifies that applications should be launched using the direct method.

-display X11-display

Indicates the display for $X 11$ output. The environment variable DISPLAY is set to $X 11$-display for the applications that are launched, and '-display X11-display' is appended to the list of arguments for xterm. The latter effect is approximately equivalent to -xtermargs "-display X11-display"; this does not affect applications launched by the direct method, but such applica- 


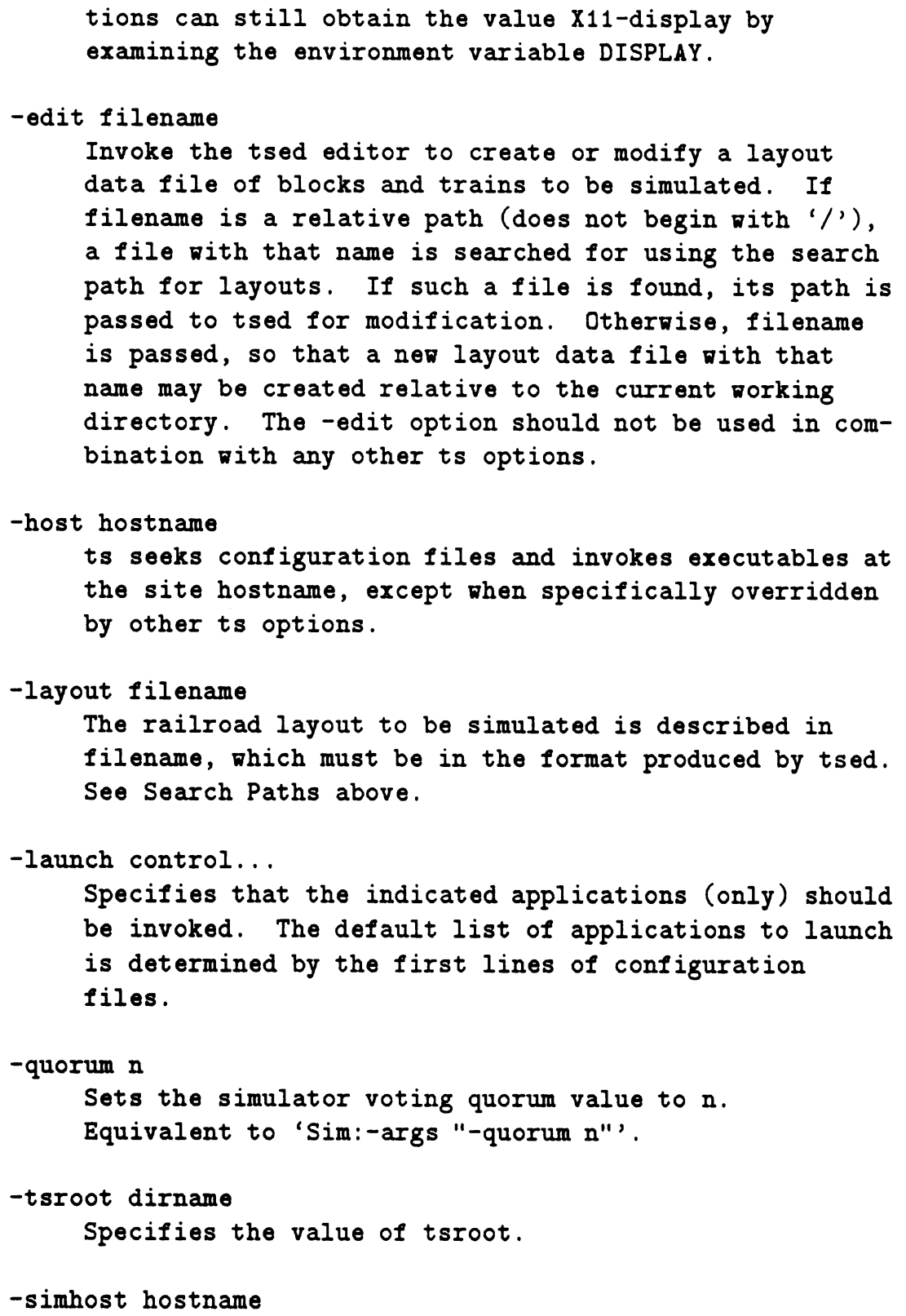




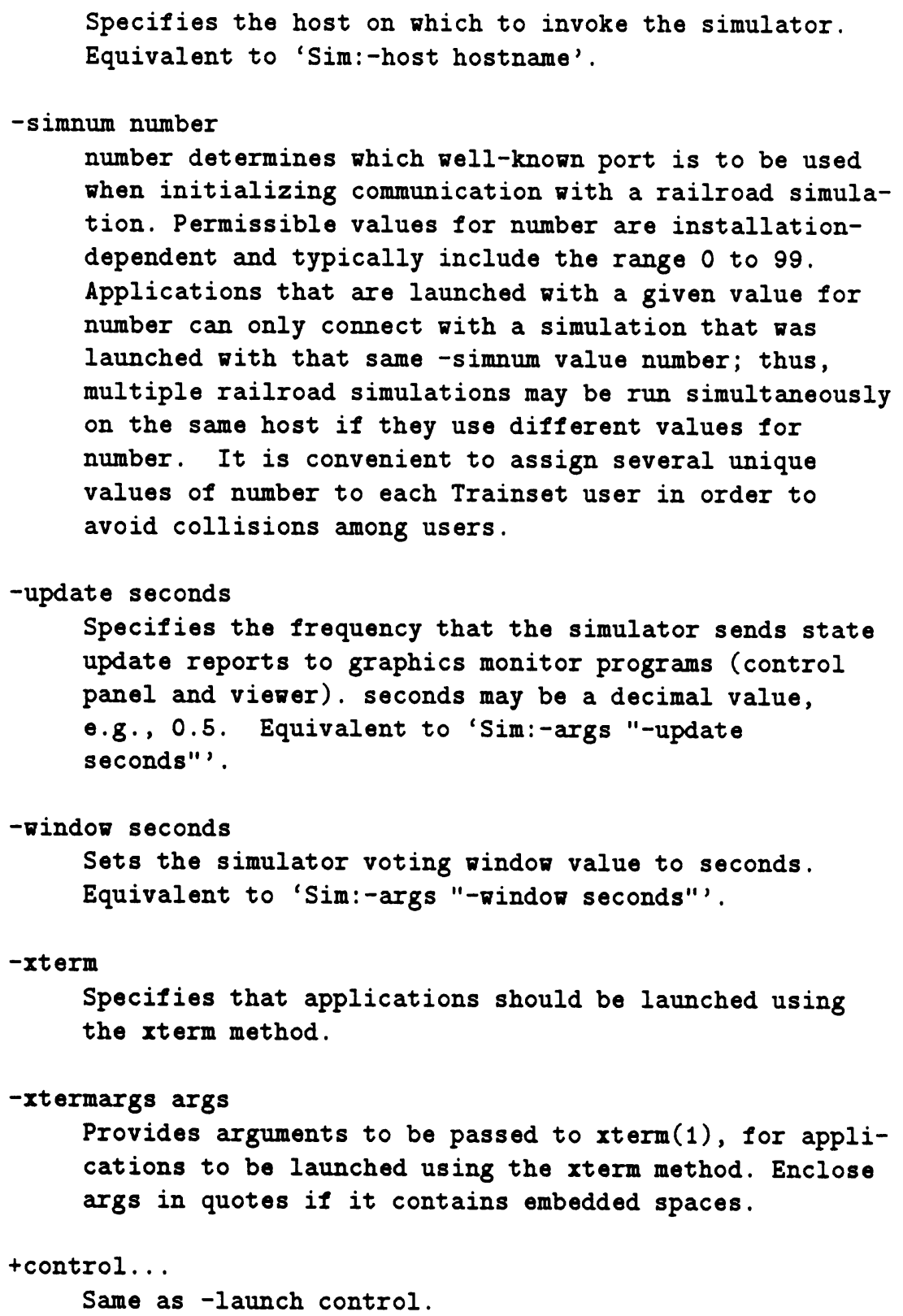


args Any options that are not listed above are passed to xterm(1) for applications launched using the xterm method. Thus, the options args is approximately equivalent to -xtermargs "args".

\section{Examples}

Start the standard programs (as listed in in configuration files) using their default methods. Use the default layout data file to define the railroad being simulated. Send all graphics output to the $X 11$ server specified in the environment variable DISPLAY, and interpret any options listed in the environment variable TSOPTS.

ts

Seek a file oval.l as described in Search Paths above for layout data files. If it is found, then start the standard programs as before, with the simulator tsim using that file oval.l to define the railroad layout.

ts -layout oval.1

Start the standard programs as before, except send output for the Panel application to thor:0 and send all other graphics output to the $X 11$ server loki:0. Send updated information to any running graphics monitor programs (e.g., Viever and Panel) every $3 / 4$ second.

ts -display loki:0 -update .75 Panel:"-display thor:0" 
$\operatorname{ts}(1)$

Launch the applications $\mathrm{S}, \mathrm{V}$ and $\mathrm{A}$ using their default

methods. Unless these control codes have been reassigned, they refer to the Simulator, Viewer and Acitest applications.

$$
\text { ts +SVA }
$$

Launch the applications $S, V$ and $A$ using the direct method. Output for the various applications will be intermingled on the screen. Since A is specified last, that application can be operated interactively. The direct method is useful for debugging new applications and for systems that do not support the program xterm(1).

$$
\text { ts +SVA -direct }
$$

Bugs

The remote invocation facility is not currently implemented.

Unpredictable results may occur if a control code is made a duplicate of itself.

See Also

tsed(1), tsim(1), tspanel(1), tsvien(1), ts.config(5), ts.remote(8). 
$\operatorname{tsed}(1)$

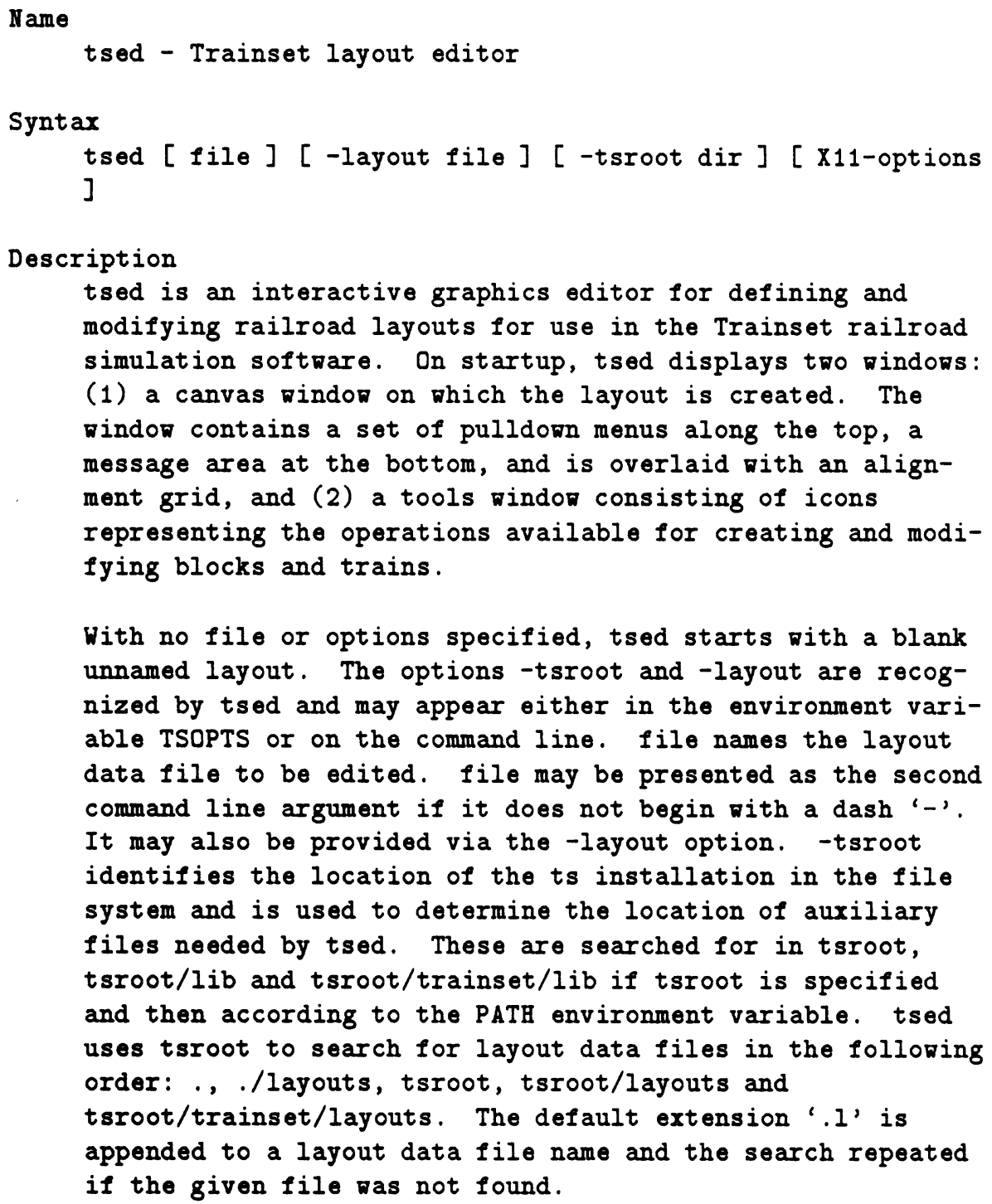

With no file or options specified, tsed starts with a blank unnamed layout. The options -tsroot and -layout are recognized by tsed and may appear either in the environment variable TSOPTS or on the command line. file names the layout data file to be edited. file may be presented as the second command line argument if it does not begin with a dash '-'. It may also be provided via the -layout option. -tsroot identifies the location of the ts installation in the file system and is used to determine the location of auxiliary files needed by tsed. These are searched for in tsroot, tsroot/lib and tsroot/trainset/lib if tsroot is specified and then according to the PATH environment variable. tsed uses tsroot to search for layout data files in the following order:.,./layouts, tsroot, tsroot/layouts and tsroot/trainset/layouts. The default extension '. I' is appended to a layout data file name and the search repeated if the given file was not found.

tsed also recognizes the standard $X 11$ application options. 
$\operatorname{tsed}(1)$

Layout Tools

The layout tools lie in two columns of icons in the tools window and are called (top to bottom, left to right) Select, Erase, Straight Block, Station Block, Arc Block 1, Arc Block 2, Cross Block, Join Block, Switch Block 1, Switch Block 2, Train, and Rotate. Select, Erase, and Rotate manipulate existing blocks. Straight Block and Station Block create linear blocks. ArC Block 1 and Arc Block 2 create circular arc blocks. Cross Block, Join Block, Switch Block 1, and Switch Block 2 create fixed size iconic blocks. The current tool is shown highlighted by inverting its colors and is set by clicking on it. Clicking the left mouse button in the canvas area invokes the current layout tool. tsed provides the following layout tools:

Select Move block labels by dragging within their boundaries. Select a block by clicking on it whether or not it's already selected and discard any other selection.

Erase Remove a block or train from a layout by clicking on it.

The four tools, Straight Block, Station Block, Arc Block 1, and Arc Block 2, create regular and station blocks using a drag technique to determine the position of the head and tail terminators of the block. If the start point or end point of the drag is within a fer pixels of the terminator of an existing block, tsed attempts to establish a connection. In such a case, tsed constrains the slope of the nev block to match the slope of the existing block at the terminator.

Straight Block Create a straight regular block. The block is constrained to be vertical, horizontal, or at + or -45 deg diagonal.

Station Block Create a station block. Created and constrained in the same way as straight block 
$\operatorname{tsed}(1)$

above.

Arc Block 1 Create a circular arc regular block. The start point of a drag determines the location of the head terminator of the block. The radius of the circular arc is two grid divisions. The extent of the arc is determined by the angle given by the head terminator and the end point of the drag. The extent of the arc is constrained to be a multiple of 45 deg.

ArC Block 2 The same as Arc Block 1 but with a radius of four grid divisions.

The four tools, Cross Block, Join Block, Switch Block 1, and Sritch Block 2, create iconic blocks, blocks that have a fixed size and shape. They are created by simply clicking the mouse, whose cursor takes the form of the icon for the current tool. Along the outer circle of such a cursor are enlarged points called hot spots at which connections with other blocks can be made. When a connection is established, the newly created iconic block is constrained so that the slope at the connecting hot spot and the slope at the existing terminator agree. tsed does not establish more than one connection when an iconic block is created.

Cross Block Create a cross block.

Join Block Create a join block.

Siitch Block 1 Create a switch block whose turn head is 45 deg counter clockwise from its straight head.

Switch Block 2 Create a switch block whose turn head is 45 deg clocknise from its straight head.

Train Create a train. The drag operation for creating a train begins at the position 
$\operatorname{tsed}(1)$

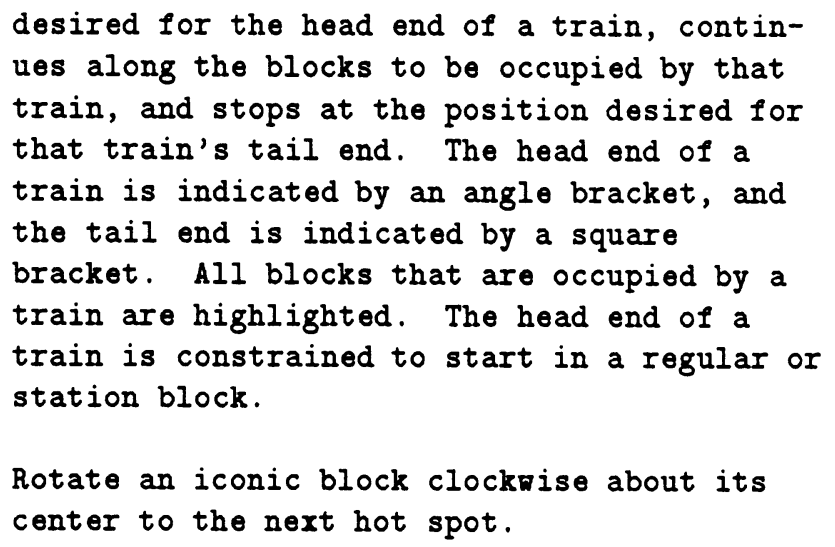

Rotate an iconic block clocknise about its center to the next hot spot.

Rotate

\section{Pulldown Menus}

The pulldown menus File and Customize lie in a menu bar along the top of the canvas window. They contain commands which you execute by pulling down the menu and releasing the mouse button on the command.

The File menu contains the following commands to operate on files :

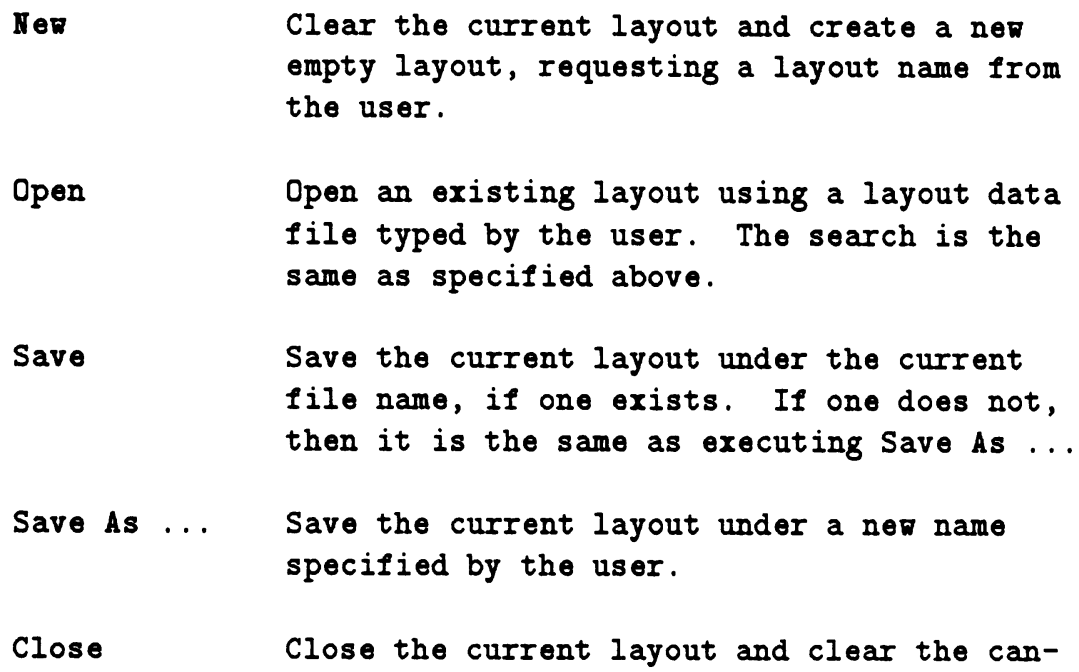


$\operatorname{tsed}(1)$

vas.

Quit Exit the application after first checking for any unsaved changes.

The Customize menu contains the following commands to alter the appearance of the current layout and the attributes of the blocks contained within:

Change Block Attributes

Change the attributes of a selected block (See Select above). If no block is selected, a warning beep is sounded.

Change Default Block Attributes

Change the default creation attributes of a block type. This is a hierarchical menu whose submenu are the various block types.

Labels

Change the appearance of the labels on the current layout. This is a hierarchical menu whose submenu entries are: Names -- Labels are block names; Speeds -- Labels indicate the minimum, maximum, and stop velocities associated with each block; Hide Labels -Hide the labels in the current layout.

Hide/Show Grid Hide (Show) the grid lines in the canvas window. Note that this does not turn the grid alignment and constraints off, it merely removes the grid from vien.

Files tsroot/lib/tsed.uid

See Also ts(1), layout data file description

Bugs / Restrictions 
$\operatorname{tsed}(1)$

Due to a bug in the DEC Xqdsg server, tsed causes the $X$ server to crash when using the ArC Block 1 and ArC Block 2 tools with $X$ servers from Ultrix 4.0 and Ultrix 4.1. There is no problem in Ultrix 4.2 and the Ultrix $4.2 X$ server can be used with Ultrix 4.0 and 4.1 . 


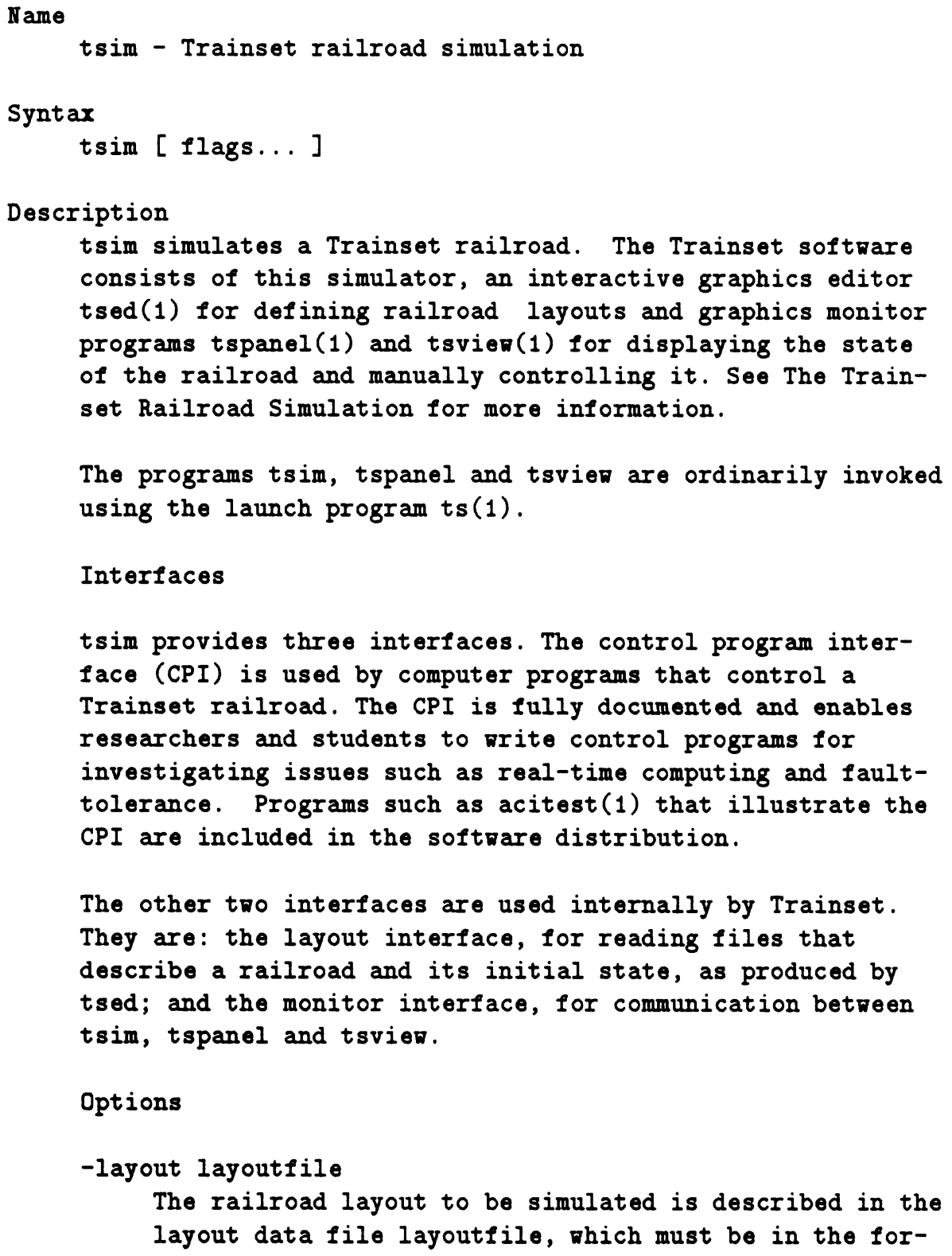

The programs tsim, tspanel and tsvien are ordinarily invoked using the launch program ts(1).

Interfaces

tsim provides three interfaces. The control program interface (CPI) is used by computer programs that control a Trainset railroad. The CPI is fully documented and enables researchers and students to write control programs for investigating issues such as real-time computing and faulttolerance. Programs such as acitest(1) that illustrate the CPI are included in the softrare distribution.

The other two interfaces are used internally by Trainset. They are: the layout interface, for reading files that describe a railroad and its initial state, as produced by tsed; and the monitor interface, for communication between tsim, tspanel and tsview.

Options

-layout layoutfile

The railroad layout to be simulated is described in the layout data file layoutfile, which must be in the for- 
$\operatorname{tsim}(1)$

mat produced by tsed. Specify a complete absolute or relative path for layoutile.

-quorum $n$

Sets the voting quorum value for this simulation to $n$.

-simnum number

Number determines which well-known ports are to be used

when other programs are initializing communication with

this simulation. Permissible values for number are installation-dependent and typically include the range 0 to 100. Multiple instances of tsim may be run simultaneously on the same host if they use different values for number.

- update seconds

Specifies the frequency that tsim is to send state update reports to graphics monitor programs. Seconds may be a decimal value, e.g., 0.5 .

- -indor seconds

Sets the voting window value for this simulation to seconds.

See Also

ts(1), tsed(1), tspanel(1), tsvien(1), acitest(1). 
tspanel(1)

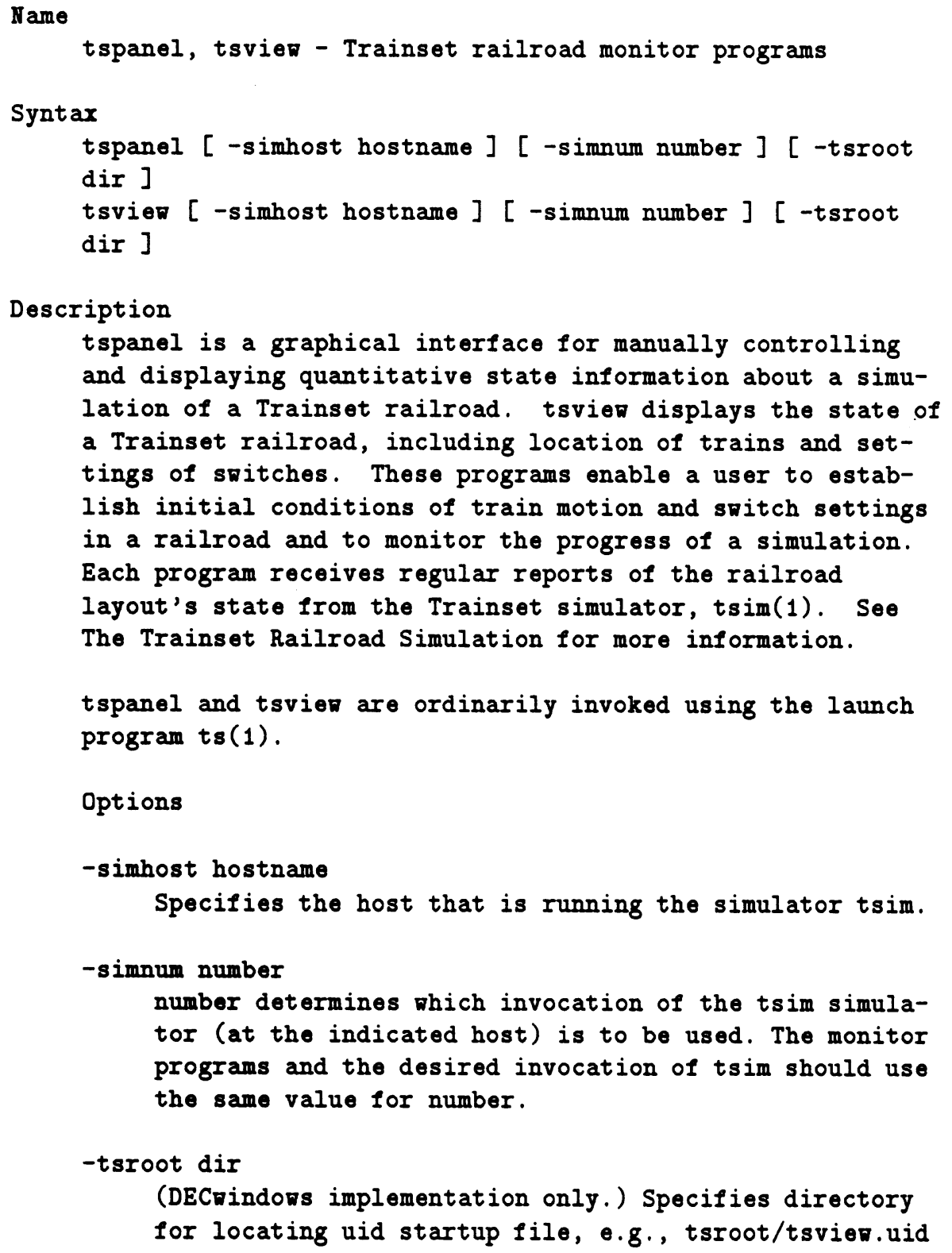


tspanel (1)

or tsroot/lib/tsvien.uid.

Limitations

Currently implemented on DECrindows only.

See Also

ts(1), tsim(1). 